TRANSACTIONS OF THE

AMERICAN MATHEMATICAL SOCIETY

Volume 348, Number 10, October 1996

\title{
MULTI-BUMP ORBITS HOMOCLINIC TO RESONANCE BANDS
}

\author{
TASSO J. KAPER AND GREGOR KOVAČIČ
}

\begin{abstract}
We establish the existence of several classes of multi-bump orbits homoclinic to resonance bands for completely-integrable Hamiltonian systems subject to small-amplitude Hamiltonian or dissipative perturbations. Each bump is a fast excursion away from the resonance band, and the bumps are interspersed with slow segments near the resonance band. The homoclinic orbits, which include multi-bump Šilnikov orbits, connect equilibria and periodic orbits in the resonance band. The main tools we use in the existence proofs are the exchange lemma with exponentially small error and the existence theory of orbits homoclinic to resonance bands which make only one fast excursion away from the resonance bands.
\end{abstract}

\section{INTRODUCTION}

Near-integrable systems occupy a central position in the theory of dynamical systems. They arise when integrable Hamiltonian systems are subjected to small conservative or dissipative perturbations. Starting from the regular motion of an integrable Hamiltonian system, which has well defined geometric features in its phase space, one addresses two main questions. The first question concerns identifying which features of the unperturbed phase space survive under given perturbations. The second question concerns identifying what possibly new structures may arise as the result of these perturbations.

The first question spawned two cornerstones of our present knowledge of dynamical systems: the Kolmogorov-Arnold-Moser theory (see Kolmogorov [32], Arnold [3], Moser [47], [48], [49]), and the persistence theory for normally hyperbolic invariant manifolds. (See Poincaré [54], Hadamard [18], Perron [53], Diliberto [8, 9], Hale [19], Kyner [40], Kupka [38], Sacker [58], [59], Anosov [1], Kurzweil [39], Jarnik and Kurzweil [27], Hirsch, Pugh, and Shub [23], Fenichel [11], [12], [13], [14], Coppel [7], Sacker and Sell [60], Katok and Hasselblatt [31].) The Kolmogorov-Arnold-Moser theory applies in the case when the perturbation is Hamiltonian, and guarantees the survival of the majority of the invariant tori that fill the phase space of the nearby integrable system. The persistence theory for normally hyperbolic invariant manifolds states the various conditions for the survival of (locally) invariant manifolds of dimension less than that of the full phase space, and also the survival of their stable and unstable manifolds. This theory applies to all small perturbations, not just Hamiltonian ones.

The Poincaré-Arnold-Melnikov method (see Poincaré [55], Melnikov [46], Arnold [2], Sotomayor [62], Holmes [24], Chow, Hale, and Mallet-Paret [6], Holmes and

Received by the editors June 1, 1994 and, in revised form, March 27, 1995.

1991 Mathematics Subject Classification. Primary 34A26, 34A47, 34C35, 34C37, 34D15.

(C)1996 American Mathematical Society 
Marsden [25, 26], Guckenheimer and Holmes [17], Robinson [56], Lerman and Umanski [42], Palmer [52], Gruendler [16], Robinson [57], Wiggins [66]), even though much less general and less important than the previous two theories, also belongs in the class of techniques that describe which structures survive in the phase space under perturbation. In particular, it provides computable conditions for the survival of some exceptional subsets of phase spaces, namely, the homoclinic orbits.

The second question is complementary to the first. One of the answers is the Aubry-Mather theory (see Aubry and LeDaeron [5], Katok [30], Mather [44], [45]), which concerns the formation of invariant Cantor sets, known as Cantori, in the context of two-dimensional, area-preserving mappings. Another answer is provided by the studies of resonance bands (see Arnold et al. [4], Guckenheimer and Holmes [17], Murdock [50]). In the case of Hamiltonian perturbations, these bands fill a part of the remaining phase space not filled by the Kolmogorov-Arnold-Moser tori. Resonance bands may also exist on lower-dimensional invariant submanifolds of the phase space. When dissipation is added to the perturbations, equilibria, periodic orbits, or invariant tori in the resonance bands, and possible homoclinic or heteroclinic connections between pairs of these objects, are often the only invariant structures robust enough to survive.

This paper discusses the existence and properties of multi-bump orbits homoclinic to resonance bands, which lie on two-dimensional, normally-hyperbolic invariant manifolds in the phase spaces of $(n+1)$-degree-of-freedom, near-integrable systems with autonomous perturbations. These orbits make finitely many excursions, or bumps, away from the resonance bands, interspersed with segments near these bands, and connect equilibria or periodic orbits that lie in these bands. In the unperturbed integrable counterparts of the systems under our investigation, the corresponding two-dimensional, normally hyperbolic invariant manifolds consist of families of periodic orbits and are connected to themselves by $(n+2)$-dimensional surfaces of homoclinic orbits. Resonance occurs if one of the periodic orbits on the normally hyperbolic invariant manifold is actually a circle of equilibria. Under small perturbations, the normally hyperbolic invariant manifold and its stable and unstable manifolds are known to persist, and the circle of equilibria bifurcates into a resonance band, which lies on the persistent hyperbolic manifold.

The main results of this paper are analytical criteria for the existence of four classes of multi-bump orbits homoclinic and heteroclinic to resonance bands, three in the case of dissipative perturbations, and one in the pure Hamiltonian case. They are described in Theorems 1, 2, 3, and 4 in section 4, respectively. In the dissipative case, the three classes of heteroclinic orbits are described as follows: Theorem 1 describes orbits that result from intersections of $(n+1)$-dimensional stable and $(n+1)$-dimensional unstable manifolds; Theorem 2 describes orbits that result from intersections of $(n+2)$-dimensional stable and $n$-dimensional unstable manifolds; and Theorem 3 describes orbits that result from intersections of $(n+2)$-dimensional stable and $(n+1)$-dimensional unstable manifolds. Finally, in the Hamiltonian case, the orbits we construct in Theorem 4 are heteroclinic connections between pairs of periodic orbits in the resonance bands, and result from the intersections of $(n+1)$-dimensional stable and unstable manifolds that are transverse inside the level surfaces of the perturbed Hamiltonian. In all cases, the orbits may also be homoclinic. 
The tools used for proving Theorems 1 through 4 are a combination of the Poincaré-Arnold-Melnikov method, rescaling used for describing resonance bands, and geometric singular perturbation theory. In particular, since the zero-perturbation limit of a resonance band is a circle of equilibria, the orbits in such a resonance band evolve on a time scale much slower than the orbits in the rest of the phase space. Due to the presence of two disparate time scales, the problem of orbits homoclinic to resonance bands is a singular perturbation problem. In particular, there exists an annular domain containing the resonance band on the persistent hyperbolic manifold, which, under appropriate rescaling, becomes a slow manifold of the type described in Fenichel [14], Jones and Kopell [28], Jones et al. [29], Lin [43], Sakamoto [61], and Tin [63, 64], and the dynamics on and near it may be approximated by an outer system. In contrast, the evolution of orbits on and near the persistent stable and unstable manifolds of this slow manifold occurs on a fast time scale. These orbits can thus be approximated by an inner system, and the relative disposition of the global stable and unstable manifolds of the persistent hyperbolic manifold may be determined using the Poincaré-Arnold-Melnikov theory. The multi-bump orbits homoclinic to resonance bands are therefore approximated by successive orbit segments of the outer and inner systems, respectively, which form so called singular transition orbits.

At the two ends of each of the multi-bump orbits homoclinic to resonance bands, the matching between the inner and outer approximations is achieved by using Fenichel's geometric singular perturbation theory in the same way as for one-bump homoclinic orbits, which is described in Kovačič [35, 36]. In fact, the results presented in the current paper are a direct extension of these one-bump results to the multi-bump case. The matching of the inner and outer approximations at the ends of the slow segments that lie in between the bumps is the new aspect that distinguishes the multi-bump case from the one-bump case. This matching is achieved with the help of a new tool, the exchange lemma with exponentially small error developed in Jones et al. [29] and Tin [63, 64], which is also built on the Fenichel theory.

The exchange lemma tracks invariant manifolds while orbits on them spend long intervals of time near the slow manifolds. The lemma states that, at the point at which such an orbit exits a small neighborhood of the slow manifold, the tracked manifold and its tangent space are transcendentally close, in terms of the small perturbation parameter, to the unstable manifold of the slow manifold and its tangent space, respectively. Thus, the exchange lemma can be used to track the stable and unstable manifolds of the annular slow manifold as they wind in and out of a small neighborhood of this slow manifold, and guarantees that they intersect under a small angle proportional to the size of the perturbation along a multi-bump twodimensional surface. The special coordinate change near the slow manifold, which recasts the equations in the Fenichel normal form (Fenichel [14]), and which makes the application of the exchange lemma possible, also makes possible further estimates of the distance between orbits on the two-dimensional intersection surfaces and the corresponding limiting singular transition orbits. These estimates enable us to use the methods of Kovačič $[35,36]$ for the matching between the inner and outer approximations at the two ends of each of the multi-bump orbits homoclinic to resonance bands.

Finally, we illustrate the theory presented in the four main theorems on the example of a Duffing oscillator coupled to an anharmonic oscillator. In particular, 
we find a plethora of multi-bump homoclinic orbits in this example, including a new class of multi-bump Silnikov orbits. We also show how the shape of a particular homoclinic orbit in each of the classes we discuss can be described by an appropriate symbol sequence of the same length as the number of bumps on the orbit.

The theory of one-bump orbits homoclinic to resonance bands has been developed in Kovačič and Wiggins [37], Kovačič [33, 34, 35], and Haller and Wiggins [21]. The existence of an entirely different class of multi-bump orbits homoclinic to slow manifolds has been proven in Haller and Wiggins [21] concurrently with the present paper. Orbits in this different class do not arise from singular transition orbits such as the ones described here. Rather than being composed of alternating slow and fast pieces, these orbits possess finitely many fast pieces that follow one after the other, since the orbit does not spend a long time near the slow manifold in between. In this sense, the two papers study complementary situations.

The paper is organized as follows. In section 2 , we formulate the problem by specifying the systems under consideration. The relevant background from the theory of resonance bands, from Fenichel's work on geometric singular perturbation theory and from the Poincaré-Arnold-Melnikov method is given in section 3. In section 4 , we state the main results of the paper in Theorems 1 through 4 . In section 5, we establish the concept of multi-bump singular intersection surfaces and modified multi-bump singular intersection surfaces, state persistence results for these surfaces under small perturbations, and also state proximity results for orbits on these persisting surfaces. In section 6 , we state the version of the exchange lemma suitable for our purposes, after putting our equations into the form of the systems treated by the exchange lemma. We prove the persistence of the multi-bump singular intersection surfaces in section 7 . In section 8 , we present the closeness result for orbits on these surfaces. In section 9, we extend the results of sections 7 and section 8 to the case of modified multi-bump singular intersection surfaces. Then in section 10, the four classes of multi-bump homoclinic orbits are established. Finally, the example is discussed in section 11.

\section{The Setup}

The systems we study have the form

$$
\begin{gathered}
\dot{x}=J D_{x} H(x, I)+\varepsilon g^{x}(x, I, \theta, \lambda), \\
\dot{I}=\varepsilon g^{I}(x, I, \theta, \lambda), \\
\dot{\theta}=D_{I} H(x, I)+\varepsilon g^{\theta}(x, I, \theta, \lambda),
\end{gathered}
$$

where $x=\left(x_{1}, \ldots, x_{2 n}\right) \in \mathbb{R}^{2 n}, I \in \mathbb{R}$, and $\theta \in S^{1}$. Furthermore, $D_{x}$ denotes the partial derivatives with respect to $x, D_{I}$ is the partial derivative with respect to

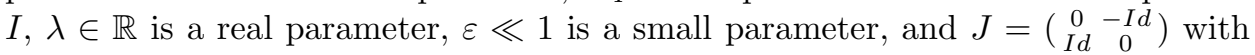
$I d$ being the $n \times n$ identity matrix. We let $\langle\cdot, \cdot\rangle$ denote the usual euclidean inner product and $\|\cdot\|$ the induced euclidean norm, as well as the corresponding matrix norm.

In the special case of a purely Hamiltonian perturbation, which arises when the entire system is derived from the Hamiltonian $\hat{H}(x, I, \theta, \lambda, \varepsilon)=H(x, I)+$ 
$\varepsilon H_{1}(x, I, \theta, \lambda)$, the system (2.1) has the form:

$$
\begin{gathered}
\dot{x}=J D_{x} H(x, I)+\varepsilon J D_{x} H_{1}(x, I, \theta, \lambda), \\
\dot{I}=-\varepsilon D_{\theta} H_{1}(x, I, \theta, \lambda), \\
\dot{\theta}=D_{I} H(x, I)+\varepsilon D_{I} H_{1}(x, I, \theta, \lambda) .
\end{gathered}
$$

The unperturbed system is obtained by setting $\varepsilon=0$ in the equations (2.1):

$$
\begin{gathered}
\dot{x}=J D_{x} H(x, I), \\
\dot{I}=0, \\
\dot{\theta}=D_{I} H(x, I) .
\end{gathered}
$$

Equation (2.3a) is a one-parameter family of Hamiltonian systems in the variable $x$ and can be analyzed independently of $\theta$. Once equation (2.3a) has been solved, equation (2.3c) can be solved by quadrature.

We make two assumptions about equations (2.3). These assumptions are needed for all of the results in this work. The first assumption concerns complete integrability and allows us to find explicit solutions for the unperturbed system:

Assumption 1. There exist two values $I_{1}$ and $I_{2}$ satisfying $I_{1}<I_{2}$ such that for all $I$ with $I_{1}<I<I_{2}$, the system (2.3a) is completely integrable; that is, there exists a smooth family of $n$ integrals of motion, $K_{1}(x, I)=H(x, I), K_{2}(x, I), \ldots$, $K_{n}(x, I)$, whose gradients $D_{x} K_{1}(x, I), D_{x} K_{2}(x, I), \ldots, D_{x} K_{n}(x, I)$ are linearly independent at all points $x$ which are not equilibria of (2.3a) and pairwise satisfy the relationship

$$
\left\langle J D_{x} K_{i}(x, I), D_{x} K_{j}(x, I)\right\rangle=0,
$$

for all $i, j=1, \ldots, n$.

The second assumption introduces the presence of homoclinic orbits in the phase space of equations (2.3):

Assumption 2. For every $I$ with $I_{1}<I<I_{2}$, equation (2.3a) possesses a hyperbolic equilibrium $x=X(I)$, which varies smoothly with $I$ and whose stable and unstable manifolds, $W^{s}(X(I))$ and $W^{u}(X(I))$, intersect along a manifold $W(X(I))$ of homoclinic orbits, connecting the equilibrium at $x=X(I)$ to itself.

One can easily show that the eigenvalues of the matrix $J D_{x}^{2} H(X(I), I)$ exist in pairs $\kappa,-\kappa$, and hence the stable and unstable manifolds $W^{s}(X(I))$ and $W^{u}(X(I))$ of the equilibrium $X(I)$ must both be $n$-dimensional. In fact, these manifolds equal the connected piece of the level set of the functions $K_{1}(x, I), \ldots, K_{n}(x, I)$ that contains the point $x=X(I)$. Thus, the stable and unstable manifolds $W^{s}(X(I))$ and $W^{u}(X(I))$ can be represented implicitly by the set of equations

$$
K_{i}(x, I)-K_{i}(X(I), I)=0, \quad i=1, \ldots, n,
$$

which hold at the point $x=X(I)$, and therefore by continuity also on $W^{s}(X(I))$ and $W^{u}(X(I))$. The homoclinic manifold $W(X(I))$ must also be $n$-dimensional, due to the linear independence of the gradients $D_{x} K_{1}(x, I), \ldots, D_{x} K_{n}(x, I)$ away 


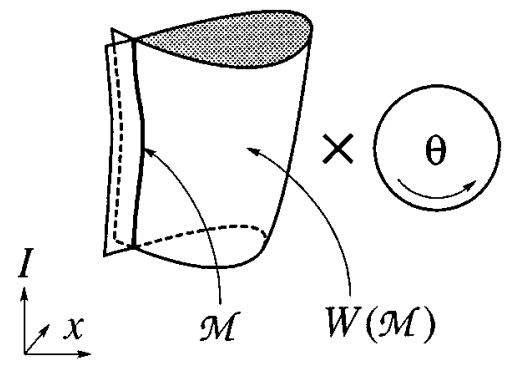

Figure 1. The invariant annulus $\mathcal{M}$ and its $n+2$-dimensional homoclinic manifold $W(\mathcal{M})$ are the cartesian product of a circle with a curve segment filled with equilibria, and its two-dimensional homoclinic manifold.

from the point $x=X(I)$. (See, for instance, the book by Wiggins [66], chapter 4, for more details on this point.)

Combining the facts that the homoclinic manifold $W(X(I))$ is $n$-dimensional and that the system $(2.3 \mathrm{a})$ is autonomous, we conclude that all the solutions on the homoclinic manifold $W(X(I))$ have a representation of the form $x^{h}\left(t-t_{0}, I, \phi\right)$, where $\phi \in \mathbb{R}^{n-1}$ is a vector of parameters. In other words, $\phi$ represents a set of $n-1$ integration constants that we obtain in the course of solving equation (2.3a) subject to the constraints (2.4). A consistent parametrization of individual orbits in the manifold $W(X(I))$ is obtained by setting $t_{0}=0$ and varying $t$ in the solution $x^{h}\left(t-t_{0}, I, \phi\right)$. Varying the parameter vector $\phi$ in this solution carries us from one orbit in the manifold $W(X(I))$ to another.

In the full $(2 n+2)$-dimensional $x-I-\theta$ phase space of the system (2.3), each equilibrium $X(I)$ corresponds to a periodic orbit $O^{I}$ parametrized by the solution

$$
x=X(I), \quad I=I, \quad \theta=D_{I} H(X(I), I) t+\theta_{0} .
$$

These periodic orbits each possess $(n+1)$-dimensional stable and unstable manifolds, $W^{s}\left(O^{I}\right)$ and $W^{u}\left(O^{I}\right)$, that are the cartesian products of the stable and unstable manifolds $W^{s}(X(I))$ and $W^{u}(X(I))$ of the equilibrium $X(I)$ and the angle $\theta$. The existence of the homoclinic manifolds $W(X(I))$ implies that the manifolds $W^{s}\left(O^{I}\right)$ and $W^{u}\left(O^{I}\right)$ coincide along an $(n+1)$-dimensional homoclinic manifold $W\left(O^{I}\right)$.

Taking the union of the orbits $O^{I}$ over all $I_{1}<I<I_{2}$, we obtain a twodimensional invariant annulus, which we denote by $\mathcal{M}$, see Figure 1 . The annulus $\mathcal{M}$ possesses $(n+2)$-dimensional stable and unstable manifolds, $W^{s}(\mathcal{M})$ and $W^{u}(\mathcal{M})$, which intersect along the $(n+2)$-dimensional homoclinic manifold $W(\mathcal{M})$. All these manifolds are the unions of the manifolds $W^{s}\left(O^{I}\right), W^{u}\left(O^{I}\right)$, and $W\left(O^{I}\right)$ along the interval $I_{1}<I<I_{2}$. The homoclinic manifold $W(\mathcal{M})$ can be represented implicitly by the set of equations (2.4) which hold on the annulus $\mathcal{M}$ at $x=X(I)$ and, hence, also on the homoclinic manifold $W(\mathcal{M})$. Furthermore, the homoclinic manifold $W(\mathcal{M})$ is parametrized by $t, I, \phi$, and $\theta_{0}$ in the solutions

$$
x=x^{h}(t, I, \phi),
$$

$$
I=I,
$$




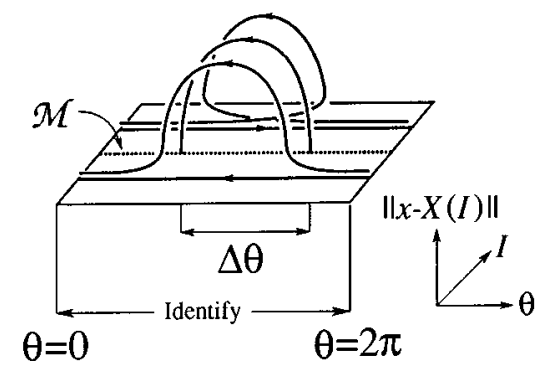

Figure 2. Geometry of manifolds homoclinic to periodic orbits and the circle of equilibria at $I=I_{0}$. Only one orbit is shown from each such manifold. All the other orbits on the same homoclinic manifold are obtained by translating those shown in the picture along the $\theta$-axis. Orbits on the manifold homoclinic to the circle of equilibria at $I=I_{0}$ are heteroclinic orbits connecting pairs of points on that circle that are $\Delta \theta$ apart.

$$
\theta=\theta^{h}(t, I)+\theta_{0}=\int_{0}^{t} D_{I} H\left(x^{h}(s, I, \phi), I\right) d s+\theta_{0} .
$$

We remark that we can prove the absence of the parameter vector $\phi$ from the expression $\theta^{h}(t, I)$ by differentiating the right-hand side of (2.6c) with respect to $\phi$ as is done in section 9 of Kovačič [36].

To conclude this section, we make two more assumptions, which are also needed for all of the results in this work. The first of these two assumptions concerns the dynamics on the annulus $\mathcal{M}$. Namely, we assume that for some $I=I_{0}$ between $I_{1}$ and $I_{2}$, the corresponding periodic orbit on the annulus $\mathcal{M}$ is an isolated circle of equilibria:

Assumption 3. For some $I_{0}$ with $I_{1}<I_{0}<I_{2}$, the frequency $D_{I} H$ has a simple zero:

$$
D_{I} H\left(X\left(I_{0}\right), I_{0}\right)=0, \quad \text { and } \quad \frac{d D_{I} H}{d I}\left(X\left(I_{0}\right), I_{0}\right) \neq 0 .
$$

As we will see in the next section, it is this circle of equilibria which perturbs to a resonance band for $\varepsilon>0$. Furthermore, we will be interested in the effect of the perturbation on the dynamics transverse to this circle of equilibria and the annulus $\mathcal{M}$. Therefore, we infer here from formulas (2.6) that pairs of points on the circle of equilibria at $I=I_{0}$, which are a fixed angle difference $\Delta \theta$ apart, are connected to each other by $n$-dimensional manifolds of heteroclinic orbits, see Figure 2. These orbits are parametrized by the variables $t$ and $\phi$ in the expressions (2.6) with $I$ fixed at $I=I_{0}$, and a fixed value of $\theta_{0}$. The angle difference $\Delta \theta$ is given by the expression

$$
\Delta \theta=\theta^{h}\left(\infty, I_{0}\right)-\theta^{h}\left(-\infty, I_{0}\right) .
$$

The final assumption concerns the resonance band, into which the circle of equilibria at $I=I_{0}$ breaks up under the perturbation. Namely, in order for this resonance band to contain only a finite number of discrete, nondegenerate equilibria, we assume 
Assumption 4. At any fixed parameter value $\lambda$, the function $g^{I}\left(X\left(I_{0}\right), I_{0}, \theta, \lambda\right)$ has only finitely many simple zeros in $\theta$ for $0 \leq \theta \leq 2 \pi$.

\section{Some Background}

For small positive $\varepsilon$, the annulus $\mathcal{M}$ and its stable and unstable manifolds $W^{s}(\mathcal{M})$ and $W^{u}(\mathcal{M})$ persist under perturbation as a locally invariant perturbed annulus, $\mathcal{M}_{\varepsilon}$, and its locally invariant perturbed stable and unstable manifolds, $W^{s}\left(\mathcal{M}_{\varepsilon}\right)$ and $W^{u}\left(\mathcal{M}_{\varepsilon}\right)$. Local invariance reflects the fact that these manifolds may leak phase points through their boundaries. Thus, the stable manifold $W^{s}\left(\mathcal{M}_{\varepsilon}\right)$ of the perturbed annulus $\mathcal{M}_{\varepsilon}$ is defined as the set of all the points that approach the annulus $\mathcal{M}_{\varepsilon}$ at a prescribed exponential rate as long as their $I$ coordinates stay within the interval $I_{1}<I<I_{2}$, and the unstable manifold $W^{u}\left(\mathcal{M}_{\varepsilon}\right)$ is defined analogously. When the perturbation is also derived from a Hamiltonian, the annulus $\mathcal{M}_{\varepsilon}$ and its stable and unstable manifolds $W^{s}\left(\mathcal{M}_{\varepsilon}\right)$ and $W^{u}\left(\mathcal{M}_{\varepsilon}\right)$ can be made invariant, see Kovačič [36]. The annulus $\mathcal{M}_{\varepsilon}$ and the manifolds $W^{s}\left(\mathcal{M}_{\varepsilon}\right)$ and $W^{u}\left(\mathcal{M}_{\varepsilon}\right)$ vary smoothly with $\varepsilon$, and collapse onto their unperturbed counterparts as $\varepsilon \rightarrow 0$. In particular, the annulus $\mathcal{M}_{\varepsilon}$ is located at the position $x=X(I)+\mathcal{O}(\varepsilon)$.

Recall that the existence of the annulus $\mathcal{M}_{\varepsilon}$ and its stable and unstable manifolds $W^{s}\left(\mathcal{M}_{\varepsilon}\right)$ and $W^{u}\left(\mathcal{M}_{\varepsilon}\right)$ is shown in the following way: First, persistence results from Fenichel $[11,12,13]$ show that the unperturbed annulus $\mathcal{M}$ persists together with its local stable and unstable manifolds, $W_{l o c}^{s}(\mathcal{M})$ and $W_{l o c}^{u}(\mathcal{M})$, that is, the connected pieces of the stable and unstable manifolds $W^{s}(\mathcal{M})$ and $W^{u}(\mathcal{M})$ that are contained in some small enough neighborhood of $\mathcal{M}$ and intersect along $\mathcal{M}$. This ensures the existence of the annulus $\mathcal{M}_{\varepsilon}$ and its local stable and unstable manifolds $W_{l o c}^{s}\left(\mathcal{M}_{\varepsilon}\right)$ and $W_{\text {loc }}^{u}\left(\mathcal{M}_{\varepsilon}\right)$. We obtain the manifolds $W^{s}\left(\mathcal{M}_{\varepsilon}\right)$ and $W^{u}\left(\mathcal{M}_{\varepsilon}\right)$ by evolving initial conditions in the local manifolds $W_{l o c}^{s}\left(\mathcal{M}_{\varepsilon}\right)$ and $W_{l o c}^{u}\left(\mathcal{M}_{\varepsilon}\right)$ in backward and forward time, respectively, see for instance Guckenheimer and Holmes [17], or Wiggins [66].

The manifolds $W^{s}\left(\mathcal{M}_{\varepsilon}\right)$ and $W^{u}\left(\mathcal{M}_{\varepsilon}\right)$ may intersect along orbits that are biasymptotic to this annulus as long as their $I$ coordinate is within $I_{1}<I<I_{2}$. We call such orbits the surviving homoclinic orbits. Their existence may be investigated by using the Poincaré-Arnold-Melnikov method. (See Poincaré [55], Melnikov [46], Arnold [2], Sotomayor [62], Holmes [24], Chow, Hale, and Mallet-Paret [6], Holmes and Marsden [25, 26], Guckenheimer and Holmes [17], Robinson [56], Lerman and Umanski [42], Palmer [52], Gruendler [16], Robinson [57], and Wiggins [66].) This method consists of calculating transverse zeros of the Melnikov vector, $\mathbf{M}\left(I, \phi, \theta_{0}, \lambda\right)$, whose $n$ components $M_{i}\left(I, \phi, \theta_{0}, \lambda\right)$ are given by the formulas

$$
M_{i}\left(I, \phi, \theta_{0}, \lambda\right)=\int_{-\infty}^{\infty}\left\langle\mathbf{n}_{i}, g\right\rangle d t,
$$

where

$$
\begin{array}{r}
\mathbf{n}_{i}=\left(D_{x} K_{i}\left(x^{h}(t, I, \phi), I\right), D_{I} K_{i}\left(x^{h}(t, I, \phi), I\right)-D_{I} K_{i}(X(I), I), 0\right), \\
i=1, \ldots, n,
\end{array}
$$

are the $n$ normals to the homoclinic manifold $W(\mathcal{M})$ and $g=\left(g^{x}, g^{I}, g^{\theta}\right)$ is the $\mathcal{O}(\varepsilon)$ perturbation part of the vector field $(2.1)$, calculated at $x=x^{h}(t, I, \phi), I$ and $\theta=\theta^{h}(t, I)+\theta_{0}$. The normals $\mathbf{n}_{i}$ can be calculated from the equations (2.4), after a short computation showing that the total derivative $\frac{d}{d I} K_{i}(X(I), I)$ equals the partial derivative $D_{I} K_{i}(X(I), I)$, see Wiggins [66], Lemma 4.1.23. 
Transverse zeros of the Melnikov vector determine which unperturbed homoclinic orbits will survive under perturbation:

Proposition 3.1. At the resonance $I=I_{0}$, if for some $\phi=\bar{\phi}, \theta_{0}=\bar{\theta}_{0}$ and $\lambda=\bar{\lambda}$ the Melnikov vector $\mathbf{M}\left(I_{0}, \bar{\phi}, \bar{\theta}_{0}, \bar{\lambda}\right)=0$, and the matrix $D_{\left(\phi, \theta_{0}\right)} \mathbf{M}\left(I_{0}, \bar{\phi}, \bar{\theta}_{0}, \bar{\lambda}\right)$ is nonsingular, then for $\varepsilon$ sufficiently small, for all I sufficiently close to the resonance, and for all $\lambda$ sufficiently close to $\bar{\lambda}$, there exists a two-dimensional intersection surface $\Sigma_{\varepsilon}^{\lambda}\left(\bar{\phi}, \bar{\theta}_{0}\right)$ of the manifolds $W^{s}\left(\mathcal{M}_{\varepsilon}\right)$ and $W^{u}\left(\mathcal{M}_{\varepsilon}\right)$ in the $x-I-\theta$ phase space. Moreover, the intersection surface $\Sigma_{\varepsilon}^{\lambda}\left(\bar{\phi}, \bar{\theta}_{0}\right)$ varies smoothly with $\varepsilon$ and $\lambda$; and, in the limit as $\varepsilon \rightarrow 0$, it tends to the surface $\Sigma^{\lambda}\left(\bar{\phi}, \bar{\theta}_{0}\right)$ that consists of the unperturbed homoclinic orbits parametrized by $t$ and $I$, with $\phi=\bar{\phi}(I, \lambda)$ and $\theta_{0}=$ $\bar{\theta}_{0}(I, \lambda)$, for I close enough to $I_{0}$ at the given $\lambda$. Finally, the manifolds $W^{s}\left(\mathcal{M}_{\varepsilon}\right)$ and $W^{u}\left(\mathcal{M}_{\varepsilon}\right)$ intersect transversely at every point of the intersection surface $\Sigma_{\varepsilon}^{\lambda}\left(\bar{\phi}, \bar{\theta}_{0}\right)$ at an angle of size $\mathcal{O}(\varepsilon)$.

This is Proposition 4.3 in Kovačič [35]. Its proof follows from the implicit function theorem and the Poincaré-Arnold-Melnikov calculation of the distance between the manifolds $W^{s}\left(\mathcal{M}_{\varepsilon}\right)$ and $W^{u}\left(\mathcal{M}_{\varepsilon}\right)$. A few more details are outlined in section 7 .

In order to focus on the resonance at $I=I_{0}$, it is advantageous to follow Guckenheimer and Holmes [17], Arnold et al. [4], or Murdock [50] in using the rescaling $I=I_{0}+\sqrt{\varepsilon} h$ on the system (2.1), which transforms this system into equations

$$
\begin{gathered}
\dot{x}=J D_{x} H\left(x, I_{0}+\sqrt{\varepsilon} h\right)+\varepsilon g^{x}\left(x, I_{0}+\sqrt{\varepsilon} h, \theta, \lambda\right), \\
\dot{h}=\sqrt{\varepsilon} g^{I}\left(x, I_{0}+\sqrt{\varepsilon} h, \theta, \lambda\right), \\
\dot{\theta}=D_{I} H\left(x, I_{0}+\sqrt{\varepsilon} h\right)+\varepsilon g^{\theta}\left(x, I_{0}+\sqrt{\varepsilon} h, \theta, \lambda\right) .
\end{gathered}
$$

Then we let $\varepsilon \rightarrow 0$, to obtain the inner system

$$
\begin{gathered}
\dot{x}=J D_{x} H\left(x, I_{0}\right), \\
\dot{h}=0, \\
\dot{\theta}=D_{I} H\left(x, I_{0}\right) .
\end{gathered}
$$

These equations are a singular limit of equations (2.1) in the sense that the circle of equilibria for (2.3) at $I=I_{0}, x=X\left(I_{0}\right), 0 \leq \theta \leq 2 \pi$, has been "blown up" into a cylinder of equilibria, $\mathcal{M}_{0}$, with $x=X\left(I_{0}\right), 0 \leq \theta \leq 2 \pi$, and arbitrary $h$. Equilibria on that cylinder are unstable, and the structure of each circle $h=$ constant and the heteroclinic orbits that connect pairs of points on it is the same (including the solutions on the heteroclinic orbits) as the structure of the circle at $I=I_{0}$ and its homoclinic manifold $W\left(X\left(I_{0}\right)\right)$, as discussed at the end of section 2 .

We denote the limit (in $x-h-\theta$ space), as $\varepsilon$ tends to zero, of the intersection surface $\Sigma_{\varepsilon}^{\lambda}\left(\bar{\phi}, \bar{\theta}_{0}\right)$ by $\Sigma_{0}^{\lambda}\left(\bar{\phi}, \bar{\theta}_{0}\right)$, see Figure 3 . This limiting one-bump homoclinic intersection surface consists of those heteroclinic orbits connecting equilibria on the $h-\theta$ cylinder $\mathcal{M}_{0}$ (under the dynamics of the inner equations (3.4)) whose $\phi$ and $\theta_{0}$ parameters equal $\phi=\bar{\phi}\left(I_{0}, \lambda\right), \theta_{0}=\bar{\theta}_{0}\left(I_{0}, \lambda\right)$. These orbits are thus explicitly given by the formula

$$
(x, h, \theta)=\left(x^{h}\left(t, I_{0}, \bar{\phi}\left(I_{0}, \lambda\right)\right), h, \theta^{h}\left(t, I_{0}\right)+\bar{\theta}_{0}\left(I_{0}, \lambda\right)\right) .
$$




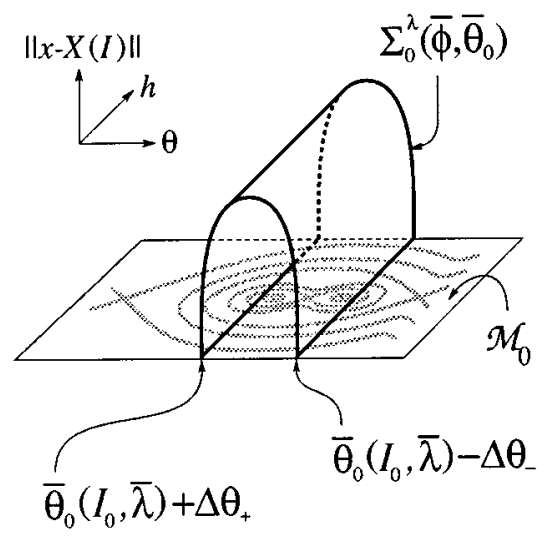

Figure 3. The singular one-bump intersection surface $\Sigma_{0}^{\lambda}\left(\bar{\phi}, \bar{\theta}_{0}\right)$ connects equilibria that lie on the line $\theta=\bar{\theta}_{0}-\Delta \theta_{-}$to those that lie on the line $\theta=\bar{\theta}_{0}+\Delta \theta_{+}$on the annulus $\mathcal{M}_{0}$. Gray curves on $\mathcal{M}_{0}$ represent the orbit structure on this annulus under the outer system (3.7).

Heteroclinic orbits comprising the surface $\Sigma_{0}^{\lambda}\left(\bar{\phi}, \bar{\theta}_{0}\right)$ emerge from the $h-\theta$ cylinder $\mathcal{M}_{0}$ along the line $\theta=\bar{\theta}_{0}\left(I_{0}, \lambda\right)-\Delta \theta_{-}$and return to $\mathcal{M}_{0}$ along the line $\theta=$ $\bar{\theta}_{0}\left(I_{0}, \lambda\right)+\Delta \theta_{+}$, where

$$
\Delta \theta_{+}=\int_{0}^{\infty} D_{I} H_{0}\left(x^{h}\left(s, I_{0}, \phi\right), I_{0}\right) d s, \quad \Delta \theta_{-}=\int_{-\infty}^{0} D_{I} H_{0}\left(x^{h}\left(s, I_{0}, \phi\right), I_{0}\right) d s .
$$

Whereas the inner equations (3.4) provide the limiting structure of the homoclinic intersection surfaces near the resonance $I=I_{0}$, they completely lose track of the dynamics on the annulus $\mathcal{M}_{\varepsilon}$ itself. In order to recover those dynamics, we restrict the equations to the annulus $\mathcal{M}_{\varepsilon}$ by requiring that the relation $x=X(I)+\mathcal{O}(\varepsilon)$ be satisfied. We also "blow up" the region near $I=I_{0}$ using the transformation $I=I_{0}+\sqrt{\varepsilon} h$ and rescale time using $\tau=\sqrt{\varepsilon} t$. Letting ' $=\frac{d}{d \tau}$, we obtain the restricted system

$$
h^{\prime}=g^{I}\left(X\left(I_{0}\right), I_{0}, \theta, \lambda\right)+\mathcal{O}(\sqrt{\varepsilon}), \quad \theta^{\prime}=\frac{d D_{I} H}{d I}\left(X\left(I_{0}\right), I_{0}\right) h+\mathcal{O}(\sqrt{\varepsilon}),
$$

and in the limit as $\varepsilon \rightarrow 0$, this system reduces to the outer system

$$
h^{\prime}=g^{I}\left(X\left(I_{0}\right), I_{0}, \theta, \lambda\right), \quad \theta^{\prime}=\frac{d D_{I} H}{d I}\left(X\left(I_{0}\right), I_{0}\right) h,
$$

which describes a resonance band. The outer system (3.7) can be derived from the rescaled Hamiltonian

$$
\mathcal{H}(h, \theta, \lambda)=\frac{1}{2} \frac{d D_{I} H}{d I}\left(X\left(I_{0}\right), I_{0}\right) h^{2}+V(\theta, \lambda),
$$

with

$$
V(\theta, \lambda)=-\int_{0}^{\theta} g^{I}\left(X\left(I_{0}\right), I_{0}, s, \lambda\right) d s
$$



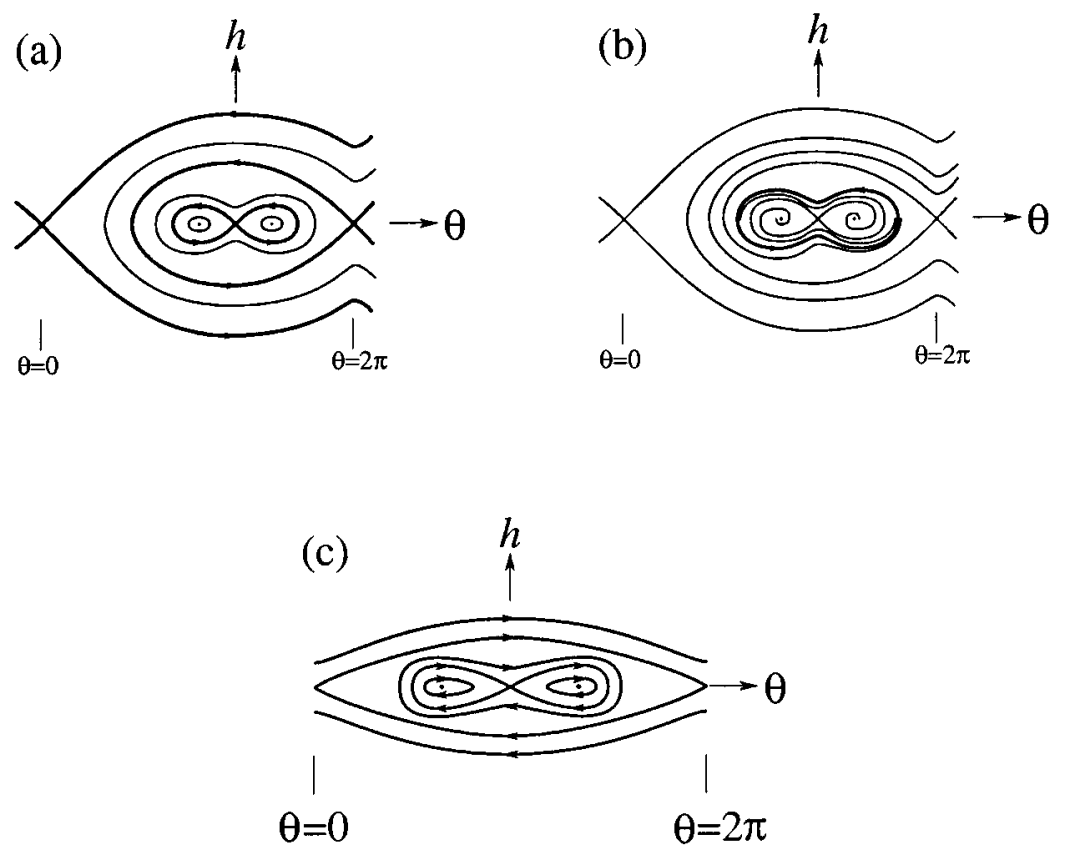

Figure 4. Typical phase portraits of the outer systems (3.7) and (3.6) for $\varepsilon=0$ and $\varepsilon>0$, respectively. Figures $4 \mathrm{a}$ and $4 \mathrm{~b}$ display the dissipative case, and Figure 4c displays the Hamiltonian case. All the points whose $\theta$ coordinates differ by a multiple of $2 \pi$ must be identified.

via the canonical formulas

$$
h^{\prime}=-D_{\theta} \mathcal{H}(h, \theta, \lambda), \quad \theta^{\prime}=D_{h} \mathcal{H}(h, \theta, \lambda) .
$$

In the Hamiltonian case, the potential $V(\theta, \lambda)$ is equal to $V(\theta, \lambda)=H_{1}\left(X\left(I_{0}\right), I_{0}, \theta\right)$, and the difference

$$
\mathcal{H}_{\varepsilon}(h, \theta, \lambda)=\frac{\left.\hat{H}\right|_{\mathcal{M}_{\varepsilon}}(h, \theta, \lambda)-H_{0}\left(X\left(I_{0}\right), I_{0}\right)}{\varepsilon}=\mathcal{H}(h, \theta, \lambda)+\mathcal{O}(\sqrt{\varepsilon})
$$

is a constant of motion for the equations (3.6), which reduces smoothly to the rescaled Hamiltonian $\mathcal{H}$ as $\sqrt{\varepsilon} \rightarrow 0$.

System (3.6) can now be analyzed with the help of (3.7). To summarize this analysis, when the perturbation part of the equations (2.1) is derived from a Hamiltonian, mild genericity assumptions guarantee that the phase portrait of the system (3.6) will have the same features as the the phase portrait of the outer system (3.7). In the dissipative case, the analysis of equations (3.6) involves the inclusion of higher-order terms in $\sqrt{\varepsilon}$. The finding of these terms is greatly facilitated by the fact that at the resonance $I=I_{0}$, the Taylor expansion $X_{\varepsilon}(I, \theta, \lambda, \varepsilon)=X(I)+\varepsilon X_{1}(I, \theta, \lambda)+\mathcal{O}\left(\varepsilon^{2}\right)$ of the perturbed annulus $\mathcal{M}_{\varepsilon}$ as a graph over the unperturbed annulus $\mathcal{M}$ can be obtained in closed form. In particular, the 
$\mathcal{O}(\varepsilon)$ term in this expansion is given by

$$
\begin{aligned}
& X_{1}\left(I_{0}, \theta, \lambda\right) \\
& \quad=\left[J D_{x}^{2} H\left(X\left(I_{0}\right), I_{0}\right)\right]^{-1}\left(g^{I}\left(X\left(I_{0}\right), I_{0}, \theta, \lambda\right) \frac{d X\left(I_{0}\right)}{d I}-g^{x}\left(X\left(I_{0}\right), I_{0}, \theta, \lambda\right)\right) .
\end{aligned}
$$

This term usually suffices for determining the dynamics of the system (3.6). Typical phase portraits of the resonance bands for the dissipative and Hamiltonian case are shown in Figure 4. We refer the reader to Kovačič [35, 36] for more details.

\section{Statement of the Main Results}

As was mentioned in the introduction, the main results paper are the criteria for the existence of $N$-bump analogs of the one-bump orbits described in Kovačič $[35,36]$. In this section, we first define the new concepts of an $N$-bump singular transition orbit and an $N$-bump singular intersection surface, and then use them to state our main Theorems 1, 2, 3, and 4 as persistence results for special types of singular transition orbits.

We begin by making two assumptions. The first assumption is

Assumption 5. The Melnikov vector $\mathbf{M}\left(I_{0}, \phi, \theta_{0}, \lambda\right)$ at the resonance vanishes simultaneously at the points $\phi=\bar{\phi}_{i}, \theta_{0}=\bar{\theta}_{0, i}, i=1, \ldots, N$, for some $\lambda=\bar{\lambda}$, with $D_{\left(\phi, \theta_{0}\right)} \mathbf{M}\left(I_{0}, \bar{\phi}_{i}, \bar{\theta}_{0, i}, \bar{\lambda}\right)$ having maximal rank for each $i$.

Under this assumption, Proposition 3.1 implies that there exist $N$ individual, not necessarily disjoint, one-bump homoclinic intersection surfaces $\Sigma_{\varepsilon}^{\lambda}\left(\bar{\phi}_{i}, \bar{\theta}_{0, i}\right), i=$ $1, \ldots, N$, for all $\lambda$ close to $\bar{\lambda}$ that limit onto the surfaces $\Sigma_{0}^{\lambda}\left(\bar{\phi}_{i}, \bar{\theta}_{0, i}\right)$ as $\varepsilon \rightarrow 0$. The second assumption is

Assumption 6. For $\lambda=\bar{\lambda}$, there exist $N-1$ orbit segments $O_{i}(\bar{\lambda}), i=2, \ldots, N$, on the annulus $\mathcal{M}_{0}$, where the endpoints of the segment $O_{i}(\bar{\lambda})$ are $d_{i}(\bar{\lambda})$ and $c_{i}(\bar{\lambda})$, respectively, and the trajectories of the outer system (3.7) flow from $d_{i}(\bar{\lambda})$ to $c_{i}(\bar{\lambda})$ in forward time. Furthermore, the line $\theta=\bar{\theta}_{0, i}\left(I_{0}, \bar{\lambda}\right)-\Delta \theta_{-}$intersects the segment $O_{i}(\bar{\lambda})$ transversely at the point $c_{i}(\bar{\lambda})$ for $i=2, \ldots, N$, and the line $\theta=$ $\bar{\theta}_{0, i}\left(I_{0}, \bar{\lambda}\right)+\Delta \theta_{+}$intersects the segment $O_{i+1}(\bar{\lambda})$ transversely at the point $d_{i+1}(\bar{\lambda})$ for $i=1, \ldots, N-1$. For all $i=2, \ldots, N-1$, the difference in the $h$-coordinates of the two points $c_{i}(\bar{\lambda})$ and $d_{i+1}(\bar{\lambda})$ is equal to zero:

$$
\begin{aligned}
\frac{1}{2} h\left(c_{i}(\bar{\lambda})\right)-\frac{1}{2} h\left(d_{i+1}(\bar{\lambda})\right) \\
=\left[\mathcal{H}\left(c_{i}(\bar{\lambda})\right)-V\left(\bar{\theta}_{0, i}-\Delta \theta_{-}\right)\right] \\
\quad-\left[\mathcal{H}\left(d_{i+1}(\bar{\lambda})\right)-V\left(\bar{\theta}_{0, i}+\Delta \theta_{+}\right)\right]=0 .
\end{aligned}
$$

This assumption implies that, for each $i=2, \ldots, N-1$, one of the heteroclinic orbits, which we label $\Gamma_{i}$, contained in the surface $\Sigma_{0}^{\lambda}\left(\bar{\phi}_{i}, \bar{\theta}_{0, i}\right)$ at $\lambda=\bar{\lambda}$, connects the two intersection points $c_{i}(\bar{\lambda})$ and $d_{i+1}(\bar{\lambda})$. Moreover, a heteroclinic orbit $\Gamma_{1}$ on the surface $\Sigma_{0}^{\lambda}\left(\bar{\phi}_{1}, \bar{\theta}_{0,1}\right)$ connects some point $c_{1}(\bar{\lambda})$ on the annulus $\mathcal{M}_{0}$ to the point $d_{2}(\bar{\lambda})$ on the segment $O_{2}(\bar{\lambda})$, and a heteroclinic orbit $\Gamma_{N}$ on the surface $\Sigma_{0}^{\lambda}\left(\bar{\phi}_{N}, \bar{\theta}_{0, N}\right)$ connects the point $c_{N}(\bar{\lambda})$ on the segment $O_{N}(\bar{\lambda})$ to some point $d_{N+1}(\bar{\lambda})$ on the annulus $\mathcal{M}_{0}$. In this case, we say that there exists an $N$-bump singular transition orbit, $\Gamma$, shown in Figure 5, which consists of the heteroclinic orbits $\Gamma_{i}$ on the 


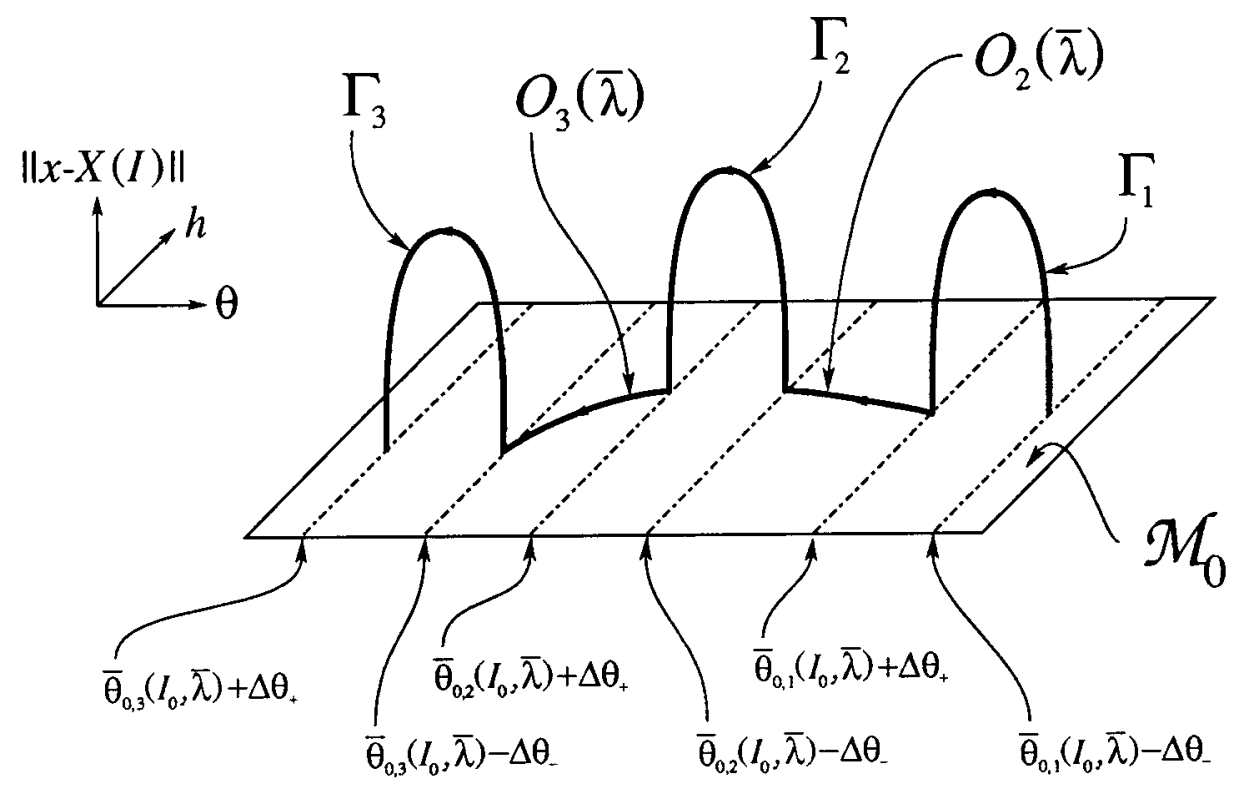

FiguRE 5. An $N$-bump singular transition orbit in the case $N=3$. This "orbit" is composed of three heteroclinic orbits $\Gamma_{1}, \Gamma_{2}$, and $\Gamma_{3}$ of the inner system (3.4), connected by two orbit segments $O_{1}(\bar{\lambda})$ and $\mathrm{O}_{2}(\bar{\lambda})$ of the outer system (3.7). The time scales of the inner and outer systems are incompatible.

surfaces $\Sigma_{0}^{\lambda}\left(\bar{\phi}_{i}, \bar{\theta}_{0, i}\right)$ for $\lambda=\bar{\lambda}$ that connect the points $c_{i}(\bar{\lambda})$ and $d_{i+1}(\bar{\lambda})$, for each $i=1, \ldots, N-1$, and the parts of the orbit segments $O_{i}(\bar{\lambda})$ between $d_{i}(\bar{\lambda})$ and $c_{i}(\bar{\lambda})$, for each $i=2, \ldots, N$. We emphasize that the orbit segments $O_{i}(\bar{\lambda})$ need not be disjoint. We call the points $c_{i}(\bar{\lambda})$ and $d_{i+1}(\bar{\lambda})$ the takeoff and landing points of the heteroclinic orbit $\Gamma_{i}$, respectively. We notice that the $h$ coordinates of the points $c_{i}(\bar{\lambda})$ and $d_{i}(\bar{\lambda})$ cannot be equal to zero if the intersections of the orbit segments $O_{i}(\bar{\lambda})$ with the lines $\theta=\bar{\theta}_{0, i}\left(I_{0}, \bar{\lambda}\right)-\Delta \theta_{-}$and $\theta=\bar{\theta}_{0, i}\left(I_{0}, \bar{\lambda}\right)+\Delta \theta_{+}$are to be transverse.

A singular transition orbit is not a true orbit, but a union of smooth curves that are orbits, or orbit segments, under the dynamics of one of two different systems that have incompatible time scales. The curves $O_{2}(\bar{\lambda}), \ldots, O_{N}(\bar{\lambda})$ are segments of orbits for the outer system (3.7) on the annulus $\mathcal{M}_{0}$, but they are curves of equilibria for the inner system (3.4). In contrast, the heteroclinic orbits $\Gamma_{1}, \ldots, \Gamma_{N}$ are orbits of the inner system (3.4), but they are not contained in the outer system, since the outer system is not defined off of the annulus $\mathcal{M}_{0}$.

By Assumption 6, for each $i=2, \ldots, N$, the intersection of the orbit segment $O_{i}(\bar{\lambda})$ with the lines $\theta=\bar{\theta}_{0, i-1}\left(I_{0}, \bar{\lambda}\right)+\Delta \theta_{+}$and $\theta=\bar{\theta}_{0, i}\left(I_{0}, \bar{\lambda}\right)-\Delta \theta_{-}$is transverse. Thus, the segment $O_{i}(\bar{\lambda})$ is contained in an open family of orbit segments of the outer system (3.7), such that each of the segments comprising this family also intersects the lines $\theta=\bar{\theta}_{0, i-1}\left(I_{0}, \bar{\lambda}\right)+\Delta \theta_{+}$and $\theta=\bar{\theta}_{0, i}\left(I_{0}, \bar{\lambda}\right)-\Delta \theta_{-}$ transversely. Since such families of open segments exist for all $i=2, \ldots, N$, it 


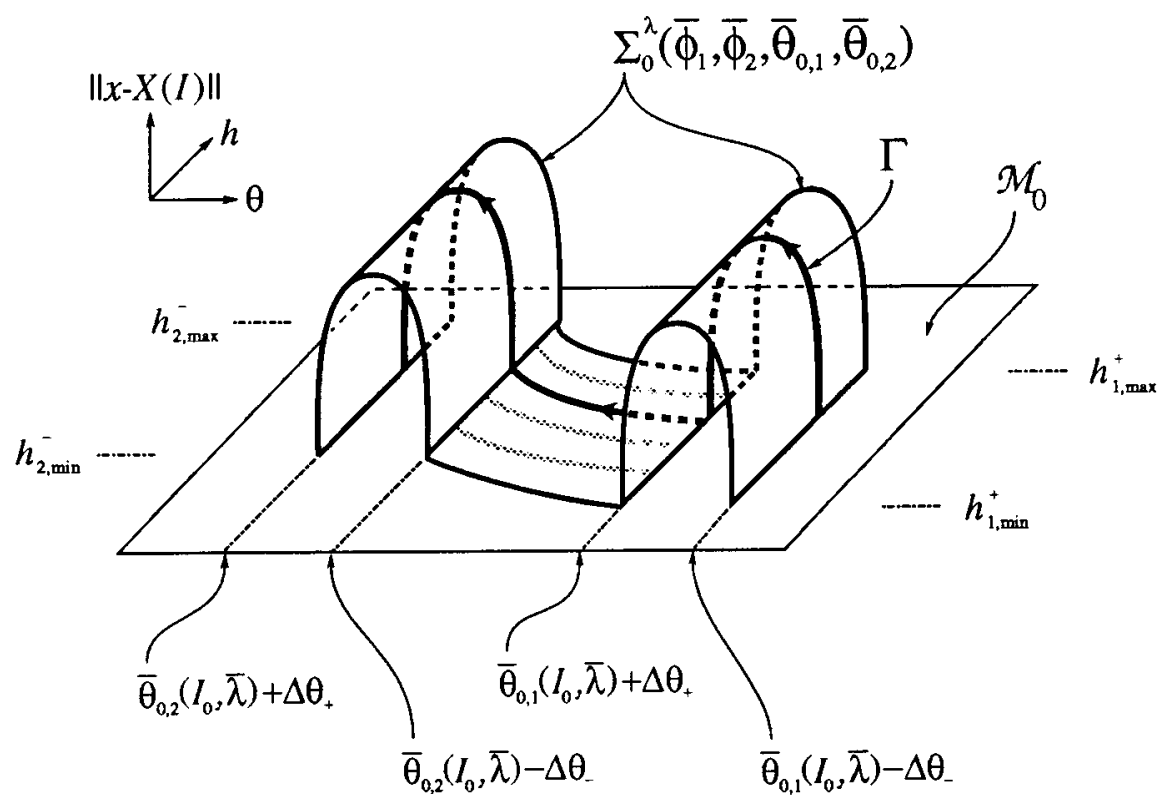

Figure $6 . \Sigma_{0}^{\lambda}\left(\bar{\phi}_{1}, \ldots, \bar{\phi}_{N}, \bar{\theta}_{0,1}, \ldots, \bar{\theta}_{0, N}\right)$ is an $N$-bump singular intersection surface, in the case $N=2$. This surface is composed of two one-bump singular intersection surfaces and adjoining orbits of the outer system. These orbits must be transverse to the takeoff line of the first and the landing line of the second of these one-bump singular intersection surfaces.

follows that there exists an open family $\Sigma_{0}^{\bar{\lambda}}\left(\bar{\phi}_{1}, \ldots, \bar{\phi}_{N}, \bar{\theta}_{0,1}, \ldots, \bar{\theta}_{0, N}\right)$ of singular transition orbits that contains the singular transition orbit $\Gamma$, and that orbits comprising $\Sigma_{0}^{\bar{\lambda}}\left(\bar{\phi}_{1}, \ldots, \bar{\phi}_{N}, \bar{\theta}_{0,1}, \ldots, \bar{\theta}_{0, N}\right)$ connect two open intervals on the lines $\theta=\bar{\theta}_{0,1}\left(I_{0}, \bar{\lambda}\right)-\Delta \theta_{-}$and $\theta=\bar{\theta}_{0, N}\left(I_{0}, \bar{\lambda}\right)+\Delta \theta_{+}$, respectively. The family $\Sigma_{0}^{\bar{\lambda}}\left(\bar{\phi}_{1}, \ldots, \bar{\phi}_{N}, \bar{\theta}_{0,1}, \ldots, \bar{\theta}_{0, N}\right)$ is a nonsmooth two-dimensional surface, which we refer to as an $N$-bump singular intersection surface, induced by the singular transition orbit $\Gamma$, see Figure 6 . By standard transversality arguments, the existence of a similar $N$-bump singular intersection surface, $\Sigma_{0}^{\lambda}\left(\bar{\phi}_{1}, \ldots, \bar{\phi}_{N}, \bar{\theta}_{0,1}, \ldots, \bar{\theta}_{0, N}\right)$, is guaranteed also for all $\lambda$ sufficiently close to $\lambda=\bar{\lambda}$.

Assumption 6 does not hold in the situation described in Theorem 2 below. Therefore, it needs to be replaced by

Assumption 7. For $\lambda=\bar{\lambda}$, there exist $N-1$ orbit segments $O_{i}(\bar{\lambda}), i=2, \ldots, N$, on the annulus $\mathcal{M}_{0}$, where the endpoints of the segment $O_{i}(\bar{\lambda})$ are $d_{i}(\bar{\lambda})$ and $c_{i}(\bar{\lambda})$, respectively, and the trajectories of the outer system (3.7) flow from $d_{i}(\bar{\lambda})$ to $c_{i}(\bar{\lambda})$ in forward time. The line $\theta=\bar{\theta}_{0, i}\left(I_{0}, \bar{\lambda}\right)-\Delta \theta_{-}$intersects the segment $O_{i}(\bar{\lambda})$ transversely at the point $c_{i}(\bar{\lambda})$ for $i=2, \ldots, N$, and the line $\theta=\bar{\theta}_{0, i}\left(I_{0}, \bar{\lambda}\right)+\Delta \theta_{+}$intersects the segment $O_{i+1}(\bar{\lambda})$ transversely at the point $d_{i+1}(\bar{\lambda})$ for $i=2, \ldots, N-1$. The $h$ coordinate of the point $d_{2}(\bar{\lambda})$ is equal to zero. Furthermore, for all $i=$ 
$2, \ldots, N-1$, the difference in the $h$-coordinates of the two points $c_{i}(\bar{\lambda})$ and $d_{i+1}(\bar{\lambda})$ is equal to zero, that is, equation (4.1) holds for $i=2, \ldots, N-1$.

This assumption is almost identical to Assumption 6, and also implies the existence of a singular transition orbit, which we call a modified singular transition orbit. The only difference between Assumptions 6 and 7 is that, since the $h$ coordinate of the point $d_{2}(\bar{\lambda})$ is equal to zero in Assumption 7 , the line $\theta=\bar{\theta}_{0,1}\left(I_{0}, \bar{\lambda}\right)+\Delta \theta_{+}$now cannot intersect the segment $O_{2}(\bar{\lambda})$ transversely. However, the form of the outer equations (3.7) implies that at all the points on the line $\theta=\bar{\theta}_{0,1}\left(I_{0}, \bar{\lambda}\right)+\Delta \theta_{+}$other than $d_{2}(\bar{\lambda})$, the orbits of the outer system (3.7) do intersect the line $\theta=\bar{\theta}_{0,1}\left(I_{0}, \bar{\lambda}\right)+\Delta \theta_{+}$transversely, but the time flow on the trajectories crossing this line moves in the opposite directions for positive and negative values of $h$, respectively. More specifically, the symmetry of the outer equations (3.7) under the transformation $(h, \tau) \rightarrow(-h,-\tau)$ implies that there exists a symmetric interval $-\bar{h}<h<\bar{h}$ on the line $\theta=\bar{\theta}_{0,1}\left(I_{0}, \bar{\lambda}\right)+\Delta \theta_{+}$such that every pair of points $\left(h, \bar{\theta}_{0,1}\left(I_{0}, \bar{\lambda}\right)+\Delta \theta_{+}\right)$and $\left(-h, \bar{\theta}_{0,1}\left(I_{0}, \bar{\lambda}\right)+\Delta \theta_{+}\right)$with $h$ in this interval is connected by a segment of an orbit of the outer system. In fact, each such segment is symmetric under reflection across the $\theta$ axis.

For values of $\lambda$ near $\lambda=\bar{\lambda}$, the line $\theta=\bar{\theta}_{0,1}\left(I_{0}, \lambda\right)+\Delta \theta_{+}$also intersects orbits transversely at all points with nonzero $h$ coordinate, and has a one-sided tangency with the trajectory that passes through the point $(h, \theta)=\left(0, \bar{\theta}_{0,1}\left(I_{0}, \lambda\right)+\Delta \theta_{+}\right)$. This tangency also implies the same properties for orbit segments of the outer system near the point $(h, \theta)=\left(0, \bar{\theta}_{0,1}\left(I_{0}, \lambda\right)+\Delta \theta_{+}\right)$as in the analogous situation at $\lambda=\bar{\lambda}$. Therefore, a unique $N$-bump modified singular transition orbit $\Gamma(\lambda)$ exists at most a distance $\mathcal{O}(\lambda-\bar{\lambda})$ away from the original $N$-bump modified singular transition orbit $\Gamma$.

In this case, we can also form a singular intersection surface, which we call the modified $N$-bump singular intersection surface, $\hat{\Sigma}_{0}^{\bar{\lambda}}\left(\bar{\phi}_{1}, \ldots, \bar{\phi}_{N}, \bar{\theta}_{0,1}, \ldots, \bar{\theta}_{0, N}\right)$, see Figure 7 . This surface is composed of all singular transition orbits that connect any open interval $-h_{0}<h<h_{0}$, with $h_{0}<\bar{h}$, on the line $\theta=\bar{\theta}_{0,1}\left(I_{0}, \bar{\lambda}\right)-\Delta \theta_{-}$to the line $\theta=\bar{\theta}_{0, N}\left(I_{0}, \lambda\right)+\Delta \theta_{+}$. However, the geometry of this modified surface is rather different from the geometry of the usual $N$-bump singular intersection surfaces. Namely, even though the transversality assumption about orbits intersecting the lines $\theta=\bar{\theta}_{0,1}\left(I_{0}, \bar{\lambda}\right)+\Delta \theta_{+}$and $\theta=\bar{\theta}_{0,2}\left(I_{0}, \bar{\lambda}\right)-\Delta \theta_{-}$implies the existence of a continuous family of orbits that contains the orbit segment $O_{2}(\bar{\lambda})$ and connects the two lines, this family is half-open, with the segment $O_{2}(\bar{\lambda})$ being on its boundary. Moreover, the fact that orbits passing through the points on the line $\theta=\bar{\theta}_{0,1}\left(I_{0}, \bar{\lambda}\right)+$ $\Delta \theta_{+}$with positive $h$ coordinate are the same as the orbits passing through the points on the line $\theta=\bar{\theta}_{0,1}\left(I_{0}, \bar{\lambda}\right)+\Delta \theta_{+}$with negative $h$ coordinate provides for a double covering of the surface $\hat{\Sigma}_{0}^{\bar{\lambda}}\left(\bar{\phi}_{1}, \ldots, \bar{\phi}_{N}, \bar{\theta}_{0,1}, \ldots, \bar{\theta}_{0, N}\right)$ from the first bump on. A modified $N$-bump singular intersection surface $\hat{\Sigma}_{0}^{\lambda}\left(\bar{\phi}_{1}, \ldots, \bar{\phi}_{N}, \bar{\theta}_{0,1}, \ldots, \bar{\theta}_{0, N}\right)$ also exists for $\lambda$ near $\lambda=\bar{\lambda}$ and, in particular, contains the $N$-bump modified singular transition orbit $\Gamma(\lambda)$.

We now state the main results of this paper in four theorems. In particular, the first two theorems concern heteroclinic orbits that exist for discrete values of the parameter $\lambda$, and the second two theorems concern heteroclinic orbits that exist on intervals of $\lambda$.

First, we consider the existence of a heteroclinic connection between either a stable periodic orbit or a saddle of the outer system to either an unstable periodic 


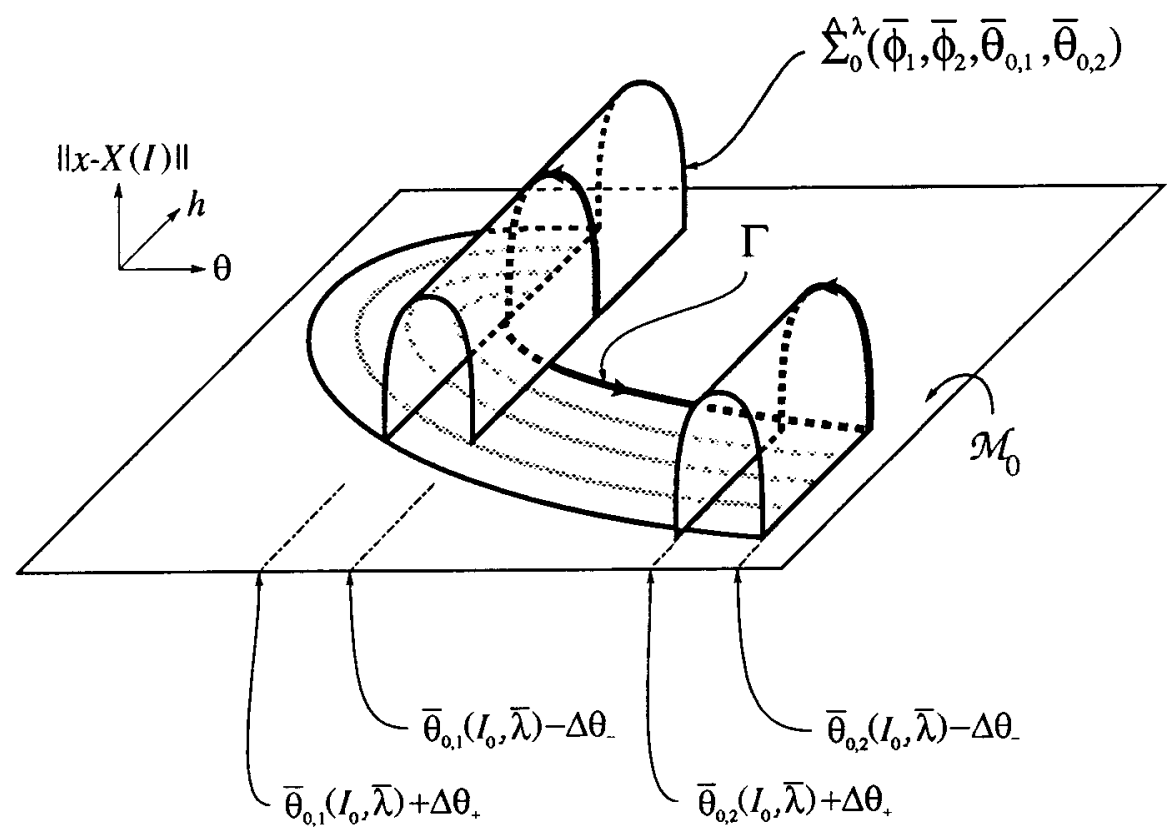

FiguRe 7. An $N$-bump modified singular transition orbit $\Gamma$, and the corresponding $N$-bump modified singular intersection surface, $\hat{\Sigma}_{0}^{\lambda}\left(\bar{\phi}_{1}, \ldots, \bar{\phi}_{N}, \bar{\theta}_{0,1}, \ldots, \bar{\theta}_{0, N}\right)$, in the case $N=2$. The slow segment of the transition orbit $\Gamma$ does not intersect the landing line of the first bump transversely, but all the neighboring orbit segments of the outer system do.

orbit or another saddle of the outer system. We assume that assumptions 1-6 hold, and state the following:

Theorem 1. For $\lambda$ near $\lambda=\bar{\lambda}$, let the curve $O_{1, \varepsilon}(\lambda)$ be either a stable periodic orbit for the restricted system (3.6) on $\mathcal{M}_{\varepsilon}$ or a (restricted) unstable manifold of a saddle $s_{1, \varepsilon}(\lambda)$ for this system, and let the curve $O_{N+1, \varepsilon}(\lambda)$ be either an unstable periodic orbit for the restricted system (3.6) on $\mathcal{M}_{\varepsilon}$ or a (restricted) stable manifold of a saddle $s_{N+1, \varepsilon}(\lambda)$ for this system. Let the line $\theta=\bar{\theta}_{0,1}\left(I_{0}, \lambda\right)-\Delta \theta_{-}$intersect the curve $O_{1,0}(\lambda)$ transversely at the point $c_{1}(\lambda)$, and let the line $\theta=\bar{\theta}_{0, N}\left(I_{0}, \lambda\right)+$ $\Delta \theta_{+}$intersect the curve $O_{N+1,0}(\lambda)$ transversely at the point $d_{N+1}(\lambda) . A t \lambda=\bar{\lambda}$, let the points $c_{1}(\bar{\lambda})$ and $d_{N+1}(\bar{\lambda})$ be connected by a singular transition orbit $\Gamma$. For $\lambda$ near $\lambda=\bar{\lambda}$, let $\mathcal{A}_{1,0}(\lambda)$ be the singular transition orbit on the singular homoclinic intersection surface $\Sigma_{0}^{\lambda}\left(\bar{\phi}_{1}, \ldots, \bar{\phi}_{N}, \bar{\theta}_{0,1}, \ldots, \bar{\theta}_{0, N}\right)$ whose takeoff point is $c_{1}(\lambda)$, and let $\mathcal{A}_{N+1,0}(\lambda)$ be the singular transition orbit whose landing point is $d_{N+1}(\lambda)$. Furthermore, assume that the singular transition orbits $\mathcal{A}_{1,0}(\lambda)$ and $\mathcal{A}_{N+1,0}(\lambda)$ pass through each other (and $\Gamma$ ) transversely as $\lambda$ passes through $\bar{\lambda}$. Then, for all small enough $\varepsilon$ and for some $\lambda=\lambda(\varepsilon)$ with $\lambda(0)=\bar{\lambda}$, there exists a heteroclinic orbit connecting either the periodic orbit $O_{1, \varepsilon}(\lambda(\varepsilon))$ or the saddle $s_{1, \varepsilon}(\lambda)$ to either the periodic orbit $O_{N+1, \varepsilon}(\lambda(\varepsilon))$ or the saddle $s_{N+1, \varepsilon}(\lambda)$. 


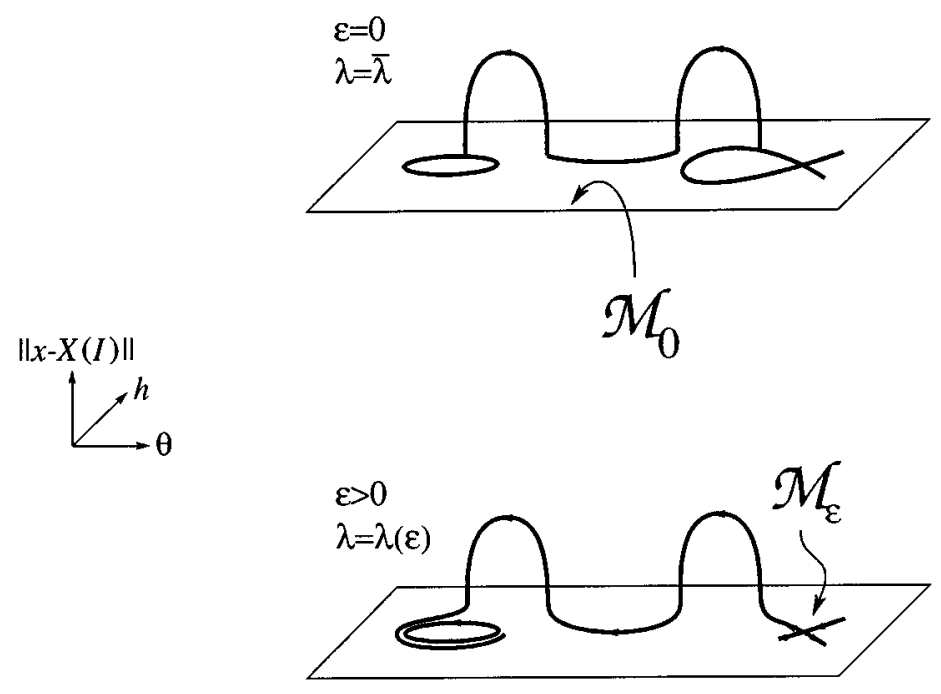

Figure 8. An illustration of Theorem 1, showing a two-bump connection between a saddle and an unstable limit cycle on the annulus $\mathcal{M}_{\varepsilon}$.

See Figure 8. The transverse passage of the singular transition orbits $\mathcal{A}_{1,0}(\lambda)$ and $\mathcal{A}_{N+1,0}(\lambda)$ through each other at $\lambda=\bar{\lambda}$ is shown in Figure 9.

Next, we describe heteroclinic orbits between either two equilibria that are sinks for the restricted system (3.6), or between a sink and a stable periodic orbit for that system, see Figure 10. Assumptions 1-5 and 7 must hold in this situation. We now state the following:

Theorem 2. Let $c_{1,0}(\lambda)$ be a center for the outer system (3.7), and let it be, at $\lambda=\bar{\lambda}$, located at

$$
\left(h\left(c_{1,0}(\bar{\lambda})\right), \theta\left(c_{1,0}(\bar{\lambda})\right)\right)=\left(0, \bar{\theta}_{0,1}\left(I_{0}, \bar{\lambda}\right)-\Delta \theta_{-}\right),
$$

with

$$
\frac{d}{d \lambda}\left[\theta\left(c_{1,0}(\lambda)\right)-\bar{\theta}_{0,1}\left(I_{0}, \lambda\right)+\Delta \theta_{-}\right] \neq 0
$$

at $\lambda=\bar{\lambda}$. Let the corresponding perturbed equilibrium $c_{1, \varepsilon}(\lambda)$ be a sink for the restricted system (3.6) for all small enough $\varepsilon$ and all $\lambda$ close enough to $\lambda=\bar{\lambda}$. Let the restricted system (3.6) on the annulus $\mathcal{M}_{\varepsilon}$ possess either another sink, $c_{\varepsilon}(\lambda)$, or a stable limit cycle, $O_{\varepsilon}(\lambda)$, and denote the associated basin of attraction by $\mathcal{B}_{\varepsilon}$. Moreover, let there exist a modified singular transition orbit connecting the point $c_{1,0}(\bar{\lambda})$ to a point $d_{N+1}(\bar{\lambda})$, and assume that the point $d_{N+1}(\bar{\lambda})$ lies in a compact domain $\mathcal{R}$ that is all contained in an open region $\mathcal{B}$, the limit as $\varepsilon \rightarrow 0$ of the basin of attraction $\mathcal{B}_{\varepsilon}$. Then, for small $\varepsilon>0$, there exists a function $\lambda=\lambda(\varepsilon)$ with $\lambda(0)=\bar{\lambda}$ and a heteroclinic orbit connecting the equilibrium $c_{1, \varepsilon}(\lambda(\varepsilon))$ to either the equilibrium $c_{\varepsilon}\left(\lambda(\varepsilon)\right.$ ), or the periodic orbit $O_{\varepsilon}(\lambda(\varepsilon))$. 

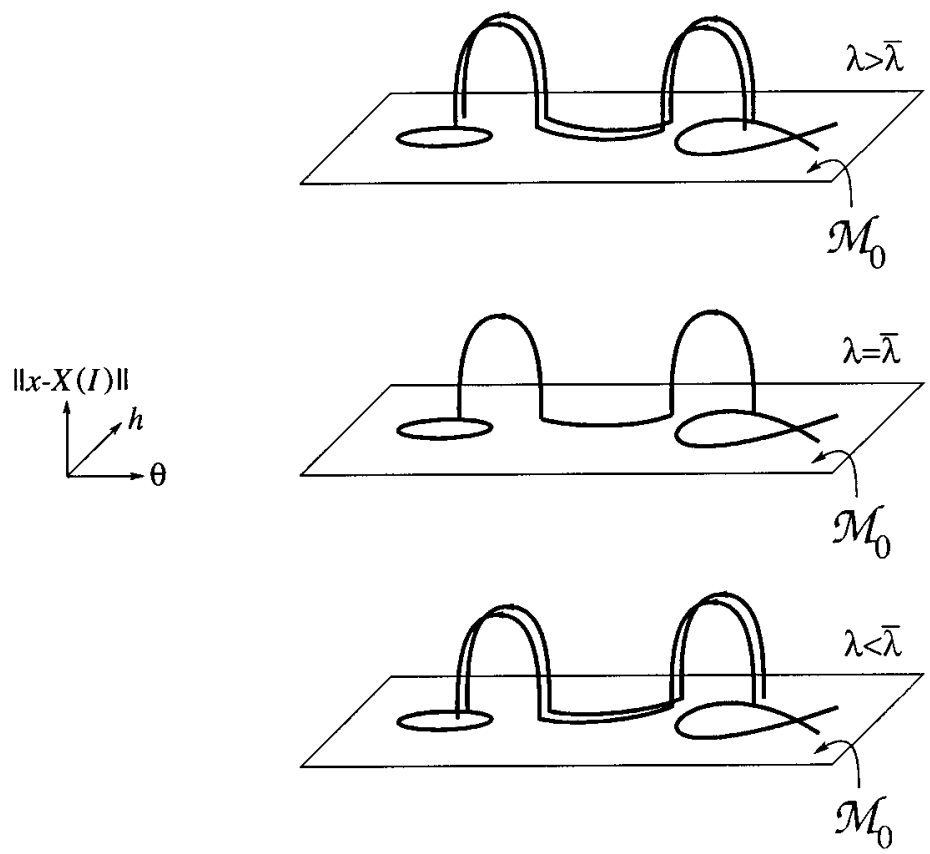

Figure 9. The singular transition orbits $\mathcal{A}_{1,0}(\lambda)$ and $\mathcal{A}_{N+1,0}(\lambda)$ pass through each other transversely as $\lambda$ passes through $\bar{\lambda}$.
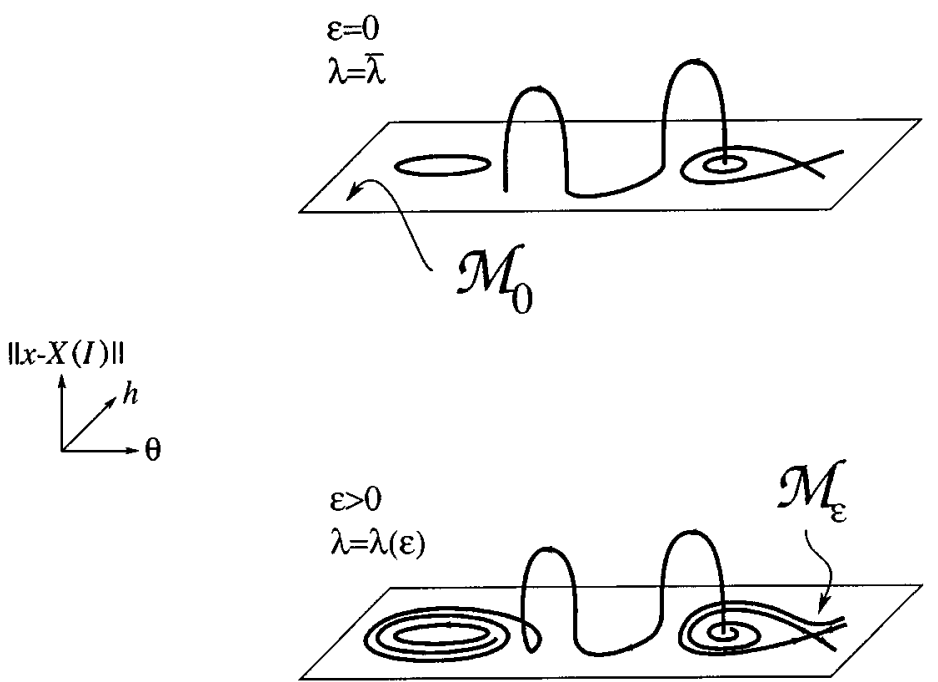

Figure 10. An illustration of Theorem 2, showing a two-bump connection between a sink and a stable limit cycle on the annulus $\mathcal{M}_{\varepsilon}$. 


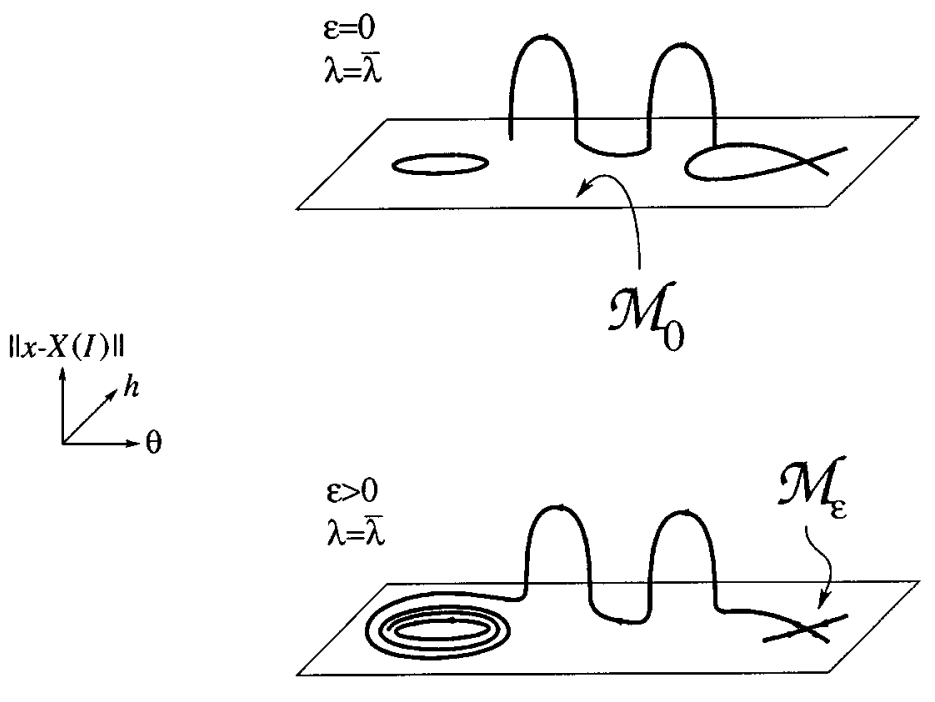

Figure 11. An illustration of Theorem 3, showing a two-bump connection between a saddle and a stable limit cycle on the annulus $\mathcal{M}_{\varepsilon}$.

Next, we find heteroclinic connections between a saddle or a stable limit cycle on the annulus $\mathcal{M}_{\varepsilon}$ and a sink or another stable limit cycle on $\mathcal{M}_{\varepsilon}$, such as the one shown in Figure 11. We assume that Assumptions 1-6 hold. We have:

Theorem 3. Let the curve $O_{1, \varepsilon}(\lambda)$ be either a stable periodic orbit on the annulus $\mathcal{M}_{\varepsilon}$, or the (restricted) unstable manifold of a saddle $s_{\varepsilon}(\lambda)$ on the annulus $\mathcal{M}_{\varepsilon}$ for all $\lambda$ near $\lambda=\bar{\lambda}$, and all small enough positive $\varepsilon$. Let the restricted system (3.6) on the annulus $\mathcal{M}_{\varepsilon}$ also possess either a sink, $c_{\varepsilon}(\lambda)$, or a stable limit cycle, $O_{\varepsilon}(\lambda)$, and denote the associated basin of attraction by $\mathcal{B}_{\varepsilon}$. At $\lambda=\bar{\lambda}$, let the curve $O_{1,0}(\bar{\lambda})$ and the line $\theta=\bar{\theta}_{0}\left(I_{0}, \lambda\right)-\Delta \theta_{-}$intersect transversely at the point $c_{1,0}(\bar{\lambda})$. Let the point $c_{1,0}(\bar{\lambda})$ be connected via a singular transition orbit $\Gamma$ to the point $d_{N+1,0}(\bar{\lambda})$. Moreover, let the point $d_{N+1,0}(\bar{\lambda})$ lie in a compact domain $\mathcal{R}$ that is all contained in an open region $\mathcal{B}$, the limit as $\varepsilon \rightarrow 0$ of the basin of attraction $\mathcal{B}_{\varepsilon}$. Then, for all small enough positive $\varepsilon$ and all $\lambda$ close enough to $\lambda=\bar{\lambda}$, there exists a heteroclinic orbit connecting either the periodic orbit $O_{1, \varepsilon}(\lambda)$ or the saddle $s_{\varepsilon}(\lambda)$ to either the equilibrium $c_{\varepsilon}(\lambda)$ or the periodic orbit $O_{\varepsilon}(\lambda)$. Moreover, the intersection of the unstable manifolds $W^{u}\left(O_{1, \varepsilon}(\lambda)\right)$ or $W^{u}\left(s_{\varepsilon}(\lambda)\right)$ with the stable manifolds $W^{s}\left(c_{\varepsilon}(\lambda)\right)$ or $W^{s}\left(O_{\varepsilon}(\lambda)\right)$ is transverse along that heteroclinic orbit.

We remark that we can describe yet different heteroclinic connections by inverting the time flow in Theorems 2 and 3.

Finally, we look at the case when the perturbed vector field (2.1) is derived from the Hamiltonian $(2.2)$, that is, $\hat{H}(x, I, \theta, \lambda, \varepsilon)=H(x, I)+\varepsilon H_{1}(x, I, \theta, \lambda)$, again assuming that Assumptions 1-6 hold. In this case a simple calculation shows that the first component of the Melnikov vector $\mathbf{M}\left(I, \phi, \theta_{0}, \lambda\right)$ equals

$$
M_{1}\left(I, \phi, \theta_{0}, \lambda\right)=H_{1}\left(X\left(I_{0}\right), I_{0}, \theta_{0}-\Delta \theta_{-}, \lambda\right)-H_{1}\left(X\left(I_{0}\right), I_{0}, \theta_{0}+\Delta \theta_{+}, \lambda\right) .
$$




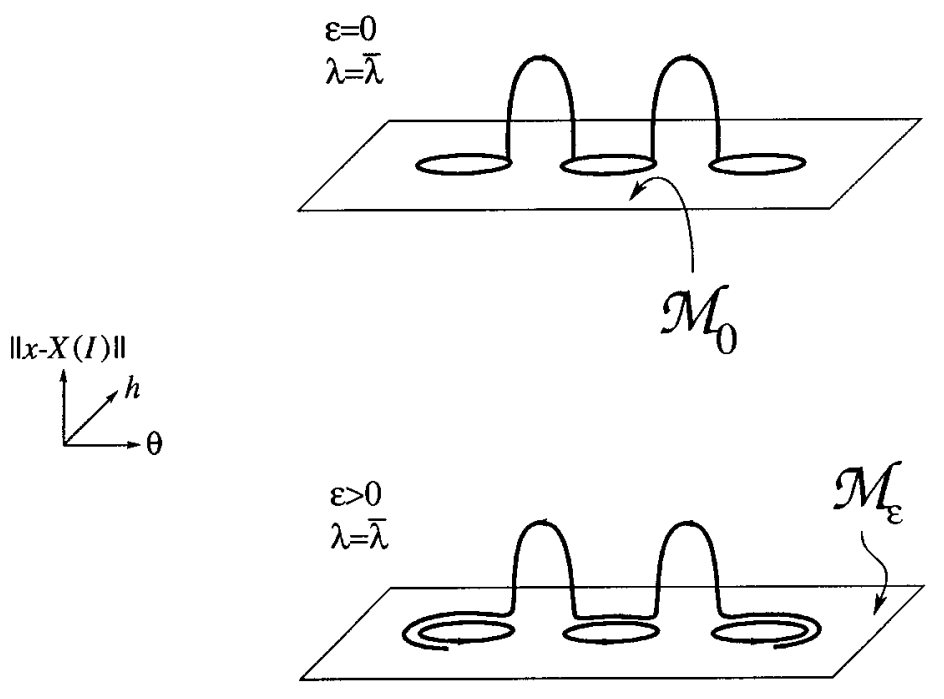

FiguRE 12. An illustration of Theorem 4, showing a three-bump connection between two periodic orbits on the annulus $\mathcal{M}_{\varepsilon}$ in the Hamiltonian case.

(See Kovačič [33, 36], or Haller and Wiggins [20].) Since we also have

$$
\mathcal{H}(h, \theta, \lambda)=\frac{1}{2} h^{2}+H_{1}\left(X\left(I_{0}\right), I_{0}, \theta, \lambda\right)
$$

in this case, we can conclude that the positions of the takeoff and landing lines $\theta=\bar{\theta}_{0, i}\left(I_{0}, \bar{\lambda}\right)-\Delta \theta_{-}$and $\theta=\bar{\theta}_{0, i}\left(I_{0}, \bar{\lambda}\right)+\Delta \theta_{+}$on the annulus $\mathcal{M}_{0}$ one determined solely from the equation

$$
\mathcal{H}\left(h, \theta-\Delta \theta_{-}, \lambda\right)-\mathcal{H}\left(h, \theta+\Delta \theta_{+}, \lambda\right)=0 .
$$

Since the rescaled Hamiltonian $\mathcal{H}(h, \theta, \lambda)$ also remains constant along the slowtime orbit segments on the annulus $\mathcal{M}_{0}$, it follows that the end points of each $N$-bump singular transition orbit on the $N$-bump singular intersection surface $\Sigma_{0}^{\lambda}\left(\bar{\phi}_{1}, \ldots, \bar{\phi}_{N}, \bar{\theta}_{0,1}, \ldots, \bar{\theta}_{0, N}\right)$ must be at the same values of $\mathcal{H}(h, \theta, \lambda)$.

Let $\Gamma$ denote a singular transition orbit connecting a pair of slow-time periodic orbits $O_{1,0}(\bar{\lambda})$ and $O_{N+1,0}(\bar{\lambda})$. From the discussion in the preceding paragraph and some simple transversality considerations, we can conclude that there exists a pair of two-parameter families,

$$
\left\{O_{1,0}^{\mu}(\lambda) \mid \mu_{1}(\lambda)<\mu<\mu_{2}(\lambda), \lambda \text { near } \bar{\lambda}\right\}
$$

and

$$
\left\{O_{N+1,0}^{\mu}(\lambda) \mid \mu_{1}(\lambda)<\mu<\mu_{2}(\lambda), \lambda \text { near } \bar{\lambda}\right\},
$$

of slow-time periodic orbits near $O_{1,0}(\bar{\lambda})$ and $O_{N+1,0}(\bar{\lambda})$, such that each pair $O_{1,0}^{\mu}(\lambda)$ and $O_{N+1,0}^{\mu}(\lambda)$ is connected by an $N$-bump singular transition orbit $\Gamma^{\mu}(\lambda)$ on the $N$-bump singular intersection surface $\Sigma_{0}^{\lambda}\left(\bar{\phi}_{1}, \ldots, \bar{\phi}_{N}, \bar{\theta}_{0,1}, \ldots, \bar{\theta}_{0, N}\right)$. Here, the parameter $\mu$ is the value of the rescaled Hamiltonian $\mathcal{H}(h, \theta, \lambda)$ along the orbits $O_{1,0}^{\mu}(\lambda)$ and $O_{N+1,0}^{\mu}(\lambda)$. 
For small positive $\varepsilon$, we conclude the following:

Theorem 4. At $\lambda=\bar{\lambda}$, assume that the line $\theta=\bar{\theta}_{0,1}\left(I_{0}, \bar{\lambda}\right)-\Delta \theta_{-}$transversely intersects a periodic orbit $O_{1,0}(\bar{\lambda})$ at the point $c_{1,0}(\bar{\lambda})$. Assume also that the line $\theta=\bar{\theta}_{0, N}\left(I_{0}, \bar{\lambda}\right)+\Delta \theta_{+}$transversely intersects a periodic orbit $O_{N+1,0}(\bar{\lambda})$ at the point $d_{N+1,0}(\bar{\lambda})$. Furthermore, assume that the points $c_{1,0}(\bar{\lambda})$ and $d_{N+1,0}(\bar{\lambda})$ are connected to each other by a singular transition orbit $\Gamma$. Then for each $\lambda$ near $\bar{\lambda}$, there exists a function $\eta(\varepsilon)$, with $\eta(\varepsilon) \rightarrow 0$ as $\varepsilon \rightarrow 0$, and two continuous families of periodic orbits,

$$
\left\{O_{1, \varepsilon}^{\mu}(\lambda) \mid \mu_{1}(\lambda)+\eta(\varepsilon)<\mu<\mu_{2}(\lambda)-\eta(\varepsilon), \lambda \text { near } \bar{\lambda}\right\}
$$

and

$$
\left\{O_{N+1, \varepsilon}^{\mu}(\lambda) \mid \mu_{1}(\lambda)+\eta(\varepsilon)<\mu<\mu_{2}(\lambda)-\eta(\varepsilon), \lambda \text { near } \bar{\lambda}\right\},
$$

such that each pair $O_{1, \varepsilon}^{\mu}(\lambda)$ and $O_{N+1, \varepsilon}^{\mu}(\lambda)$ is connected by an $N$-bump heteroclinic orbit lying on the $N$-bump intersection surface $\Sigma_{\varepsilon}^{\lambda}\left(\bar{\phi}_{1}, \ldots, \bar{\phi}_{N}, \bar{\theta}_{0,1}, \ldots, \bar{\theta}_{0, N}\right)$. The value of the parameter $\mu$ along the orbits $O_{1, \varepsilon}^{\mu}(\lambda)$ and $O_{N+1, \varepsilon}^{\mu}(\lambda)$ is equal to the value of the constant $\mathcal{H}_{\varepsilon}(h, \theta, \lambda)$ along these orbits, and also to the value of the rescaled Hamiltonian $\mathcal{H}(h, \theta, \lambda)$ along the orbits $O_{1,0}^{\mu}(\lambda)$ and $O_{N+1,0}^{\mu}(\lambda)$. As $\varepsilon \rightarrow 0$, the union of the orbits $O_{1, \varepsilon}^{\mu}(\lambda)$ and $O_{N+1, \varepsilon}^{\mu}(\lambda)$ and the connecting heteroclinic orbit collapses onto the union of the curves $O_{1,0}^{\mu}(\lambda)$ and $O_{N+1,0}^{\mu}(\lambda)$ and the singular transition orbit $\Gamma^{\mu}(\lambda)$. Moreover the intersection of the unstable manifold $W^{u}\left(O_{1, \varepsilon}^{\mu}(\lambda)\right)$ with the stable manifold $W^{s}\left(O_{N+1, \varepsilon}^{\mu}(\lambda)\right)$ along the heteroclinic orbit that connects the orbits $O_{1, \varepsilon}^{\mu}(\lambda)$ and $O_{N+1, \varepsilon}^{\mu}(\lambda)$ is transverse inside the corresponding level surface of the perturbed Hamiltonian $\hat{H}(x, I, \theta, \lambda, \varepsilon)$.

See Figure 12.

The rest of this paper will be devoted to proving the above four theorems.

\section{Multi-Bump Homoclinic Intersection Surfaces}

The proofs of the four theorems stated in the previous section are carried out in two steps. The first step consists of establishing the persistence under perturbation of the $N$-bump singular intersection surfaces and $N$-bump modified singular intersection surfaces as true $N$-bump intersection surfaces between the stable and unstable manifolds $W^{s}\left(\mathcal{M}_{\varepsilon}\right)$ and $W^{u}\left(\mathcal{M}_{\varepsilon}\right)$ of the annulus $\mathcal{M}_{\varepsilon}$. The second step consists of establishing proximity results for orbits on these surfaces. The first step is carried out in Lemmas 1 and 3, and the second step is carried out in Lemmas 2 and 4 , all of which are stated below. The two steps are combined with the results of Kovačič [35] and [36] to prove Theorems 1-4 in section 6 .

We first show that an $N$-bump singular intersection surface gives rise to a true $N$-bump intersection surface of the manifolds $W^{s}\left(\mathcal{M}_{\varepsilon}\right)$ and $W^{u}\left(\mathcal{M}_{\varepsilon}\right)$ that exists nearby for sufficiently small $\varepsilon$ in

Lemma 1. If for $\lambda=\bar{\lambda}$ and $\varepsilon=0$ there exists an $N$-bump singular transition orbit, $\Gamma$, in the $x-h-\theta$ space, then, for all $\varepsilon>0$ and $\lambda$ near $\lambda=\bar{\lambda}$, there exists a two-dimensional, $N$-bump intersection surface $\Sigma_{\varepsilon}^{\lambda}\left(\bar{\phi}_{1}, \ldots, \bar{\phi}_{N}, \bar{\theta}_{0,1}, \ldots, \bar{\theta}_{0, N}\right)$ of the manifolds $W^{s}\left(\mathcal{M}_{\varepsilon}\right)$ and $W^{u}\left(\mathcal{M}_{\varepsilon}\right)$. This intersection surface varies smoothly with $\lambda$ and $\varepsilon$ for $\varepsilon>0$, and limits onto the $N$-bump singular intersection surface 


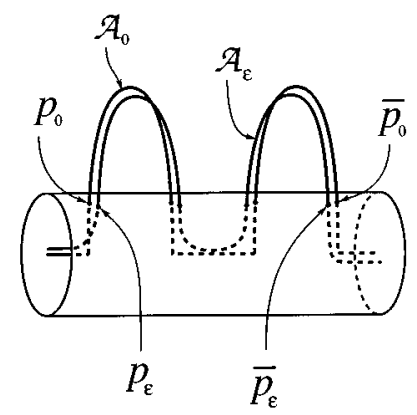

FiguRE 13. In Lemma 2 , the orbit $\mathcal{A}_{\varepsilon}$ on the $N$-bump intersection surface $\Sigma_{\varepsilon}^{\lambda}\left(\bar{\phi}_{1}, \ldots, \bar{\phi}_{N}, \bar{\theta}_{0,1}, \ldots, \bar{\theta}_{0, N}\right)$ stays close to some singular transition orbit $\mathcal{A}_{0}$ on the $N$-bump singular intersection surface $\Sigma_{0}^{\lambda}\left(\bar{\phi}_{1}, \ldots, \bar{\phi}_{N}, \bar{\theta}_{0,1}, \ldots, \bar{\theta}_{0, N}\right)$ as a curve between the first and the last pairs of points where these two curves exit the small neighborhood $V_{\delta}$ of the annulus $\mathcal{M}_{0}$.

$\Sigma_{0}^{\lambda}\left(\bar{\phi}_{1}, \ldots, \bar{\phi}_{N}, \bar{\theta}_{0,1}, \ldots, \bar{\theta}_{0, N}\right)$ as $\varepsilon \rightarrow 0$. Moreover, the intersection of the manifolds $W^{s}\left(\mathcal{M}_{\varepsilon}\right)$ and $W^{u}\left(\mathcal{M}_{\varepsilon}\right)$ along the surface $\Sigma_{\varepsilon}^{\lambda}\left(\bar{\phi}_{1}, \ldots, \bar{\phi}_{N}, \bar{\theta}_{0,1}, \ldots, \bar{\theta}_{0, N}\right)$ is transverse.

The proof of this lemma will be given at the end of section 7 .

We now focus on the dynamics on the homoclinic intersection surfaces. In particular, we show that a trajectory on a true intersection surface, which starts close to a singular transition orbit on a singular homoclinic intersection surface, remains close to that singular orbit as a curve. Before stating this result, we define the neighborhood $V_{\delta}$ as the set of points in the $x-h-\theta$ space with $\left\|x-X\left(I_{0}\right)\right\|<\delta$, and the boundary $\partial V_{\delta}$ as the set of points with $\left\|x-X\left(I_{0}\right)\right\|=\delta$. We now establish

Lemma 2. Let there be a singular transition orbit $\Gamma$ at $\lambda=\bar{\lambda}$. For $\lambda$ near $\bar{\lambda}$, let $\mathcal{A}_{\varepsilon}$ be an orbit on the $N$-bump homoclinic intersection surface

$$
\Sigma_{\varepsilon}^{\lambda}\left(\bar{\phi}_{1}, \ldots, \bar{\phi}_{N}, \bar{\theta}_{0,1}, \ldots, \bar{\theta}_{0, N}\right)
$$

that pierces the boundary $\partial V_{\delta}$ at a point $p_{\varepsilon}$ near the line $\theta=\bar{\theta}_{0,1}\left(I_{0}, \bar{\lambda}\right)-\Delta \theta_{-}$ on the annulus $\mathcal{M}_{0}$. Furthermore, let $\mathcal{A}_{0}$ be any singular transition orbit on the singular homoclinic intersection surface $\Sigma_{0}^{\lambda}\left(\bar{\phi}_{1}, \ldots, \bar{\phi}_{N}, \bar{\theta}_{0,1}, \ldots, \bar{\theta}_{0, N}\right)$ that pierces the boundary $\partial V_{\delta}$ at a point $p_{0}$ a distance $\mathcal{O}(\sqrt{\varepsilon})$ away from $p_{\varepsilon}$. Finally, let $\bar{p}_{0}$ be the point where the orbit $\mathcal{A}_{0}$ pierces the boundary $\partial V_{\delta}$ near the line $\theta=\bar{\theta}_{0, N}\left(I_{0}, \bar{\lambda}\right)+\Delta \theta_{+}$ on the annulus $\mathcal{M}_{0}$. Then the orbit $\mathcal{A}_{\varepsilon}$ stays within $\mathcal{O}(\sqrt{\varepsilon})$ of the singular transition orbit $\mathcal{A}_{0}$, except at the corners of the orbit $\mathcal{A}_{0}$, until it reenters the neighborhood $V_{\delta}$ at the point $\bar{p}_{\varepsilon}$ on the boundary $\partial V_{\delta}$ a distance $\mathcal{O}(\sqrt{\varepsilon})$ away from the point $\bar{p}_{0}$. Moreover, there exists a function $\delta(\varepsilon)$ such that $\delta(\varepsilon) \rightarrow 0$ as $\varepsilon \rightarrow 0$ and the entire piece of the orbit $\mathcal{A}_{\varepsilon}$ between the points $p_{\varepsilon}$ and $\bar{p}_{\varepsilon}$ stays within $\mathcal{O}(\delta(\varepsilon))$ of the singular transition orbit $\mathcal{A}_{0}$, even near the corners of $\mathcal{A}_{0}$.

For an illustration, see Figure 13. We prove this lemma in section 9.

To conclude this section, we now state the analogous lemmas for the case of a modified singular transition orbit. Their proofs are given in section 9. Persistence of modified $N$-bump singular intersection surfaces is given by 


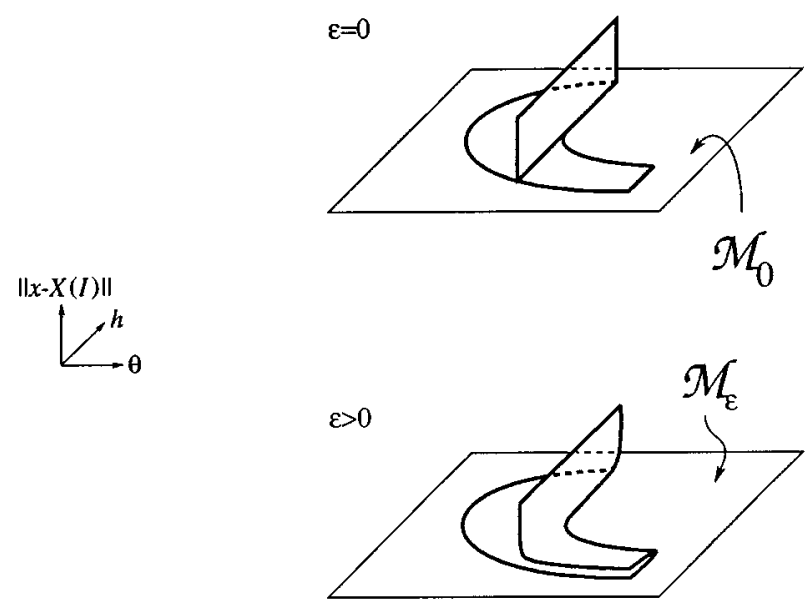

Figure 14. From the landing line of the first bump of the $N$-bump modified singular intersection surface $\hat{\Sigma}_{0}^{\lambda}\left(\bar{\phi}_{1}, \ldots, \bar{\phi}_{N}, \bar{\theta}_{0,1}, \ldots, \bar{\theta}_{0, N}\right)$ on, the singular intersection surface $\hat{\Sigma}_{\varepsilon}^{\lambda}\left(\bar{\phi}_{1}, \ldots, \bar{\phi}_{N}, \bar{\theta}_{0,1}, \ldots, \bar{\theta}_{0, N}\right)$ consists of two sheets that meet along a fold.

Lemma 3. If for $\lambda=\bar{\lambda}$ and $\varepsilon=0$ there exists a modified $N$-bump singular transition orbit, $\Gamma$, in the $x-h-\theta$ space, then, for all $\varepsilon>0$ and $\lambda$ near $\lambda=\bar{\lambda}$, there exists a two-dimensional, $N$-bump intersection surface $\hat{\Sigma}_{\varepsilon}^{\lambda}\left(\bar{\phi}_{1}, \ldots, \bar{\phi}_{N}, \bar{\theta}_{0,1}, \ldots, \bar{\theta}_{0, N}\right)$ of the manifolds $W^{s}\left(\mathcal{M}_{\varepsilon}\right)$ and $W^{u}\left(\mathcal{M}_{\varepsilon}\right)$. This intersection surface varies smoothly with $\lambda$ and $\varepsilon$ for $\varepsilon>0$, and limits onto the modified $N$-bump singular intersection surface $\hat{\Sigma}_{0}^{\lambda}\left(\bar{\phi}_{1}, \ldots, \bar{\phi}_{N}, \bar{\theta}_{0,1}, \ldots, \bar{\theta}_{0, N}\right)$ as $\varepsilon \rightarrow 0$. Moreover, the intersection of the manifolds $W^{s}\left(\mathcal{M}_{\varepsilon}\right)$ and $W^{u}\left(\mathcal{M}_{\varepsilon}\right)$ along the surface $\hat{\Sigma}_{\varepsilon}^{\lambda}\left(\bar{\phi}_{1}, \ldots, \bar{\phi}_{N}, \bar{\theta}_{0,1}, \ldots, \bar{\theta}_{0, N}\right)$ is transverse.

We will call the surface $\hat{\Sigma}_{\varepsilon}^{\lambda}\left(\bar{\phi}_{1}, \ldots, \bar{\phi}_{N}, \bar{\theta}_{0,1}, \ldots, \bar{\theta}_{0, N}\right)$ a modified $N$-bump homoclinic intersection surface. We remark that near the singular orbit $\Gamma$, the surface $\hat{\Sigma}_{\varepsilon}^{\lambda}\left(\bar{\phi}_{1}, \ldots, \bar{\phi}_{N}, \bar{\theta}_{0,1}, \ldots, \bar{\theta}_{0, N}\right)$ has a fold, and from the first bump on, two sheets of this surface stay $\mathcal{O}\left(e^{\frac{-c}{\sqrt{\varepsilon}}}\right)$ close to each other so that $\hat{\Sigma}_{\varepsilon}^{\lambda}\left(\bar{\phi}_{1}, \ldots, \bar{\phi}_{N}, \bar{\theta}_{0,1}, \ldots, \bar{\theta}_{0, N}\right)$ is globally not a graph over the modified $N$-bump singular intersection surface $\hat{\Sigma}_{0}^{\lambda}\left(\bar{\phi}_{1}, \ldots, \bar{\phi}_{N}, \bar{\theta}_{0,1}, \ldots, \bar{\theta}_{0, N}\right)$. As $\varepsilon \rightarrow 0$, these two sheets of the surface

$$
\hat{\Sigma}_{\varepsilon}^{\lambda}\left(\bar{\phi}_{1}, \ldots, \bar{\phi}_{N}, \bar{\theta}_{0,1}, \ldots, \bar{\theta}_{0, N}\right)
$$

collapse onto the doubly covered sheet of the surface

$$
\hat{\Sigma}_{0}^{\lambda}\left(\bar{\phi}_{1}, \ldots, \bar{\phi}_{N}, \bar{\theta}_{0,1}, \ldots, \bar{\theta}_{0, N}\right),
$$

see Figure 14.

Proximity of orbits on the modified $N$-bump intersection surfaces is given by

Lemma 4. Let there be a modified singular transition orbit $\Gamma$ at $\lambda=\bar{\lambda}$. For $\lambda$ near $\bar{\lambda}$, let $\mathcal{A}_{\varepsilon}$ be an orbit on the modified $N$-bump homoclinic intersection surface

$$
\hat{\Sigma}_{\varepsilon}^{\lambda}\left(\bar{\phi}_{1}, \ldots, \bar{\phi}_{N}, \bar{\theta}_{0,1}, \ldots, \bar{\theta}_{0, N}\right)
$$


that pierces the boundary $\partial V_{\delta}$ at a point $p_{\varepsilon}$ near the line $\theta=\bar{\theta}_{0,1}\left(I_{0}, \bar{\lambda}\right)-\Delta \theta_{-}$on the annulus $\mathcal{M}_{0}$. Furthermore, let $\mathcal{A}_{0}$ be any singular transition orbit on the modified singular homoclinic intersection surface $\hat{\Sigma}_{0}^{\lambda}\left(\bar{\phi}_{1}, \ldots, \bar{\phi}_{N}, \bar{\theta}_{0,1}, \ldots, \bar{\theta}_{0, N}\right)$ that pierces the boundary $\partial V_{\delta}$ at a point $p_{0}$ a distance $\mathcal{O}(\sqrt{\varepsilon})$ away from $p_{\varepsilon}$. Finally, let $\bar{p}_{0}$ be the point where the orbit $\mathcal{A}_{0}$ pierces the boundary $\partial V_{\delta}$ near the line $\theta=\bar{\theta}_{0, N}\left(I_{0}, \bar{\lambda}\right)+\Delta \theta_{+}$ on the annulus $\mathcal{M}_{0}$. Then there exists a function $\delta(\varepsilon)$, such that $\delta(\varepsilon) \rightarrow 0$ as $\varepsilon \rightarrow 0$, and that the orbit $\mathcal{A}_{\varepsilon}$ stays within $\mathcal{O}(\delta(\varepsilon))$ of the singular transition orbit $\mathcal{A}_{0}$, until it reenters the neighborhood $V_{\delta}$ at the point $\bar{p}_{\varepsilon}$ on the boundary $\partial V_{\delta}$ a distance $\mathcal{O}(\delta(\varepsilon))$ away from the point $\bar{p}_{0}$.

Since the statement of Lemma 4 appears almost indistinguishable from the statement of Lemma 2, it seems appropriate at this point to spell out the similarities and differences beeen these two lemmas. In particular, both lemmas establish closeness results between true and singular $N$-bump transition orbits. These lemmas differ, however, in the order of the closeness that they imply. For true and singular $N$-bump transition orbits $\mathcal{A}_{\varepsilon}$ and $\mathcal{A}_{0}$ on true and singular $N$-bump intersection surfaces $\Sigma_{\varepsilon}^{\lambda}\left(\bar{\phi}_{1}, \ldots, \bar{\phi}_{N}, \bar{\theta}_{0,1}, \ldots, \bar{\theta}_{0, N}\right)$ and $\Sigma_{0}^{\lambda}\left(\bar{\phi}_{1}, \ldots, \bar{\phi}_{N}, \bar{\theta}_{0,1}, \ldots, \bar{\theta}_{0, N}\right)$, respectively, Lemma 2 gives $\mathcal{O}(\sqrt{\varepsilon})$ closeness everywhere except near the corners of the singular orbit $\mathcal{A}_{0}$. In contrast, lemma 4 only gives $\mathcal{O}(\delta(\varepsilon))$ closeness between true and singular transition orbits $\mathcal{A}_{\varepsilon}$ and $\mathcal{A}_{0}$ on true and singular modified $N$-bump intersection surfaces $\hat{\Sigma}_{\varepsilon}^{\lambda}\left(\bar{\phi}_{1}, \ldots, \bar{\phi}_{N}, \bar{\theta}_{0,1}, \ldots, \bar{\theta}_{0, N}\right)$ and $\hat{\Sigma}_{0}^{\lambda}\left(\bar{\phi}_{1}, \ldots, \bar{\phi}_{N}, \bar{\theta}_{0,1}, \ldots, \bar{\theta}_{0, N}\right)$. The weaker estimate in Lemma 4 stems from the fact that if the orbit $\mathcal{A}_{\varepsilon}$ is sufficiently close to the fold of the surface $\hat{\Sigma}_{\varepsilon}^{\lambda}\left(\bar{\phi}_{1}, \ldots, \bar{\phi}_{N}, \bar{\theta}_{0,1}, \ldots, \bar{\theta}_{0, N}\right)$, then one cannot in general establish $\mathcal{O}(\sqrt{\varepsilon})$ closeness between the orbits $\mathcal{A}_{\varepsilon}$ and $\mathcal{A}_{0}$, not even away from the corners of the singular orbit $\mathcal{A}_{0}$.

\section{The Fenichel Normal Form and the Exchange Lemma}

One of the main results that we need in the proof of Lemmas 1 and 3 is the exchange lemma with exponentially small error, proven in Jones et al. [29] and Tin $[63,64]$. In addition to stating in this section the version of the lemma we need, we put the equations governing our systems near the slow manifold $\mathcal{M}_{\varepsilon}$ into the form suitable for applying the exchange lemma, and we establish some technical estimates that we will repeatedly use in the forthcoming sections.

The transformation of our system is achieved in two steps, first a direct coordinate change to obtain a normal form, and then a simplification of that normal form. The first step is provided by the following:

Proposition 6.1. In a neighborhood of the perturbed annulus $\mathcal{M}_{\varepsilon}$, there exist smooth local coordinates

$$
\begin{array}{ll}
a=a(x, h, \theta, \lambda, \sqrt{\varepsilon}), & b=b(x, h, \theta, \lambda, \sqrt{\varepsilon}), \\
\chi=\chi(x, h, \theta, \lambda, \sqrt{\varepsilon}), & \psi=\psi(x, h, \theta, \lambda, \sqrt{\varepsilon}),
\end{array}
$$

with $a, b \in \mathbb{R}^{n}$ and $\chi, \psi \in \mathbb{R}$, such that equations (3.3) can be written in the normal form

$$
\begin{gathered}
\dot{a}=\left[A(a, b)+\sqrt{\varepsilon} A_{1}(a, b, \chi, \psi, \lambda, \sqrt{\varepsilon})\right] a, \\
\dot{b}=\left[B(a, b)+\sqrt{\varepsilon} B_{1}(a, b, \chi, \psi, \lambda, \sqrt{\varepsilon})\right] b, \\
\dot{\chi}=\sqrt{\varepsilon}\left[C(\chi, \psi, \lambda, \varepsilon)+\left\langle a, C_{1}(a, b, \chi, \psi, \lambda, \sqrt{\varepsilon}) b\right\rangle\right],
\end{gathered}
$$




$$
\dot{\psi}=\langle a, \Phi(a, b) b\rangle+\sqrt{\varepsilon}\left[\Psi(\chi, \psi, \lambda, \varepsilon)+\left\langle a, \Psi_{1}(a, b, \chi, \psi, \lambda, \sqrt{\varepsilon}) b\right\rangle\right] .
$$

Here $A(a, b), \quad B(a, b), \quad \Phi(a, b), \quad A_{1}(a, b, \chi, \psi, \lambda, \sqrt{\varepsilon}), \quad B_{1}(a, b, \chi, \psi, \lambda, \sqrt{\varepsilon})$, $C_{1}(a, b, \chi, \psi, \lambda, \sqrt{\varepsilon})$, and $\Psi_{1}(a, b, \chi, \psi, \lambda, \sqrt{\varepsilon})$ are matrix-valued, and $C(\chi, \psi, \lambda, \varepsilon)$ and $\Psi(\chi, \psi, \lambda, \varepsilon)$ are scalar-valued functions which depend smoothly on the coordinates $a, b, \chi$, and $\psi$, and the parameters $\lambda$ and $\sqrt{\varepsilon}$. Moreover, the eigenvalues of the matrix $A(0,0)$ coincide with those eigenvalues of the matrix $J D_{x}^{2} H\left(X\left(I_{0}\right), I_{0}\right)$ whose real parts are positive, and the eigenvalues of the matrix $B(0,0)$ coincide with those eigenvalues of the matrix $J D_{x}^{2} H\left(X\left(I_{0}\right), I_{0}\right)$ whose real parts are negative.

The proof of Proposition 6.1 relies crucially on the following theorem from Fenichel [14], which we state below without proof in a form adapted to the needs of the present paper. The theorem is stated for the local stable manifold $W_{l o c}^{s}\left(\mathcal{M}_{\varepsilon}\right)$ of the annulus $\mathcal{M}_{\varepsilon}$. The statement for the local unstable manifold $W_{\text {loc }}^{u}\left(\mathcal{M}_{\varepsilon}\right)$ is identical except for time reversal.

Proposition 6.2. For all small enough $\varepsilon$, the local stable manifold $W_{\text {loc }}^{s}\left(\mathcal{M}_{\varepsilon}\right)$ of the invariant annulus $\mathcal{M}_{\varepsilon}$ is foliated by a family of disjoint n-dimensional manifolds called stable fibers. These stable fibers have the following additional properties:

1. They form a locally positively invariant family; that is, the image (under the forward time flow) of any stable fiber is contained in a stable fiber as long as this image is contained in the local stable manifold $W_{\text {loc }}^{s}\left(\mathcal{M}_{\varepsilon}\right)$.

2. Each stable fiber pierces the annulus $\mathcal{M}_{\varepsilon}$ transversely inside the manifold $W_{\text {loc }}^{s}\left(\mathcal{M}_{\varepsilon}\right)$ in precisely one point, called its base point.

3. As the base points on the annulus $\mathcal{M}_{\varepsilon}$ move under the dynamics of the vector field (3.3), the stable fibers move along with their base points and contract exponentially towards their base points in forward time as long as the base points stay in $\mathcal{M}_{\varepsilon}$. The rate of this exponential contraction is at least $e^{-\kappa t}$, where $\kappa$ is any number strictly less than the smallest real part of all the positive eigenvalues of equation (2.3a) at $I=I_{0}$ (or, equivalently, the inner equation (3.4a)) linearized around $x=X\left(I_{0}\right)$.

4. The family of stable fibers varies smoothly with $\sqrt{\varepsilon}, \lambda$, and any other parameters in the problem.

5. For $\varepsilon=0$, that is, for system (3.4), the stable fibers are precisely the local stable manifolds of the equilibria on the $h-\theta$ annulus $\mathcal{M}_{0}$.

Proof of Proposition 6.1. We begin by recalling the structure of the inner system (3.4), in particular the fact that the equation for the $x$ coordinate is decoupled from the rest of the system, and that the $\theta$ coordinate can be obtained by a quadrature. This structure suggests that we first transform the $x$ coordinate in a small neighborhood of the annulus $\mathcal{M}_{0}$, that is, in a small neighborhood of the equilibrium at $x=X\left(I_{0}\right)$ of the equation (3.4a). We translate this equilibrium to the origin, and then replace $x-X\left(I_{0}\right)$ by two sets of coordinates, $a=\left(a_{1}, \ldots, a_{n}\right)$ and $b=\left(b_{1}, \ldots, b_{n}\right)$, such that the hyperplanes $a=0$ and $b=0$ coincide with the local stable and unstable manifolds of the origin $a=b=0$ in that neighborhood. Thus, equations (3.4) may be rewritten as

$$
\begin{aligned}
& \dot{a}=A(a, b) a, \\
& \dot{b}=B(a, b) b,
\end{aligned}
$$




$$
\begin{gathered}
\dot{h}=0, \\
\dot{\theta}=\Theta_{a}(a, b) a+\Theta_{b}(a, b) b,
\end{gathered}
$$

where $A(a, b)$, and $B(a, b)$ are matrix-valued, and $\Theta_{a}(a, b)$ and $\Theta_{b}(a, b)$ are vectorvalued. The form of equation (6.2d) follows from the fact that $\dot{\theta}=0$ for $a=b=0$.

The coordinate change from the $x-h-\theta$ to the $a-b-h-\theta$ coordinates is in fact a composition of a linear transformation and a transformation that leaves the linear parts of the coordinates unchanged, so that the eigenvalues of the linearized equations (6.2a) and (6.2b) coincide with the eigenvalues of the matrix $J D_{x}^{2} H\left(X\left(I_{0}\right), I_{0}\right)$. More precisely, the eigenvalues of the matrix $A(0,0)$ are exactly the eigenvalues of $J D_{x}^{2} H\left(X\left(I_{0}\right), I_{0}\right)$ with positive real parts, and the eigenvalues of the matrix $B(0,0)$ are exactly the eigenvalues of $J D_{x}^{2} H\left(X\left(I_{0}\right), I_{0}\right)$ with negative real parts.

We next replace the angle $\theta$ by another angle $\psi$, which is constant along orbits in the hyperplanes $a=0$ and $b=0$, so that system (6.2) becomes

$$
\begin{gathered}
\dot{a}=A(a, b) a, \\
\dot{b}=B(a, b) b, \\
\dot{h}=0, \\
\dot{\psi}=\langle a, \Phi(a, b) b\rangle
\end{gathered}
$$

for some smooth matrix-valued function $\Phi(a, b)$. This is done in a smooth fashion in the following way. We consider the stable and unstable fibers described in Proposition 6.2. Recall from this proposition that the stable fibers are precisely the stable manifolds of the equilibria on the annulus $\mathcal{M}_{0}$ at $a=b=0$, and that the unstable fibers are the unstable manifolds of these equilibria. Also, observe that $h$ is constant under the dynamics of equations (6.2), and these equations are invariant under translations in $\theta$. Hence, the stable fibers can be expressed in the form $\theta=\psi+b f_{b}(b)$, and the unstable fibers can be expressed in the form $\theta=\psi+a f_{a}(a)$ with some smooth functions $f_{a}(a)$ and $f_{b}(b)$, where $\psi$ is the $\theta$ coordinate of the base point of the fiber. At any point not on the stable and unstable manifolds $a=0$ and $b=0$, we define the angle $\psi$ by $\psi=\theta-a f_{a}(a)-b f_{b}(b)$. Since the base points of the stable and unstable fibers are equilibria of system (6.2), we must have $\dot{\psi}=0$ on the hyperplanes $a=0$ and $b=0$. Hence the form of equation (6.3d).

For small nonzero $\sqrt{\varepsilon}$, Proposition 6.2 implies that we can choose in a smooth fashion new coordinates

$$
\begin{aligned}
a_{\varepsilon} & =a_{\varepsilon}(a, b, h, \psi, \lambda, \sqrt{\varepsilon})=a+\mathcal{O}(\sqrt{\varepsilon}), \\
b_{\varepsilon} & =b_{\varepsilon}(a, b, h, \psi, \lambda, \sqrt{\varepsilon})=b+\mathcal{O}(\sqrt{\varepsilon}), \\
\chi_{\varepsilon} & =\chi_{\varepsilon}(a, b, h, \psi, \lambda, \sqrt{\varepsilon})=h+\mathcal{O}(\sqrt{\varepsilon}), \\
\psi_{\varepsilon} & =\psi_{\varepsilon}(a, b, h, \psi, \lambda, \sqrt{\varepsilon})=\psi+\mathcal{O}(\sqrt{\varepsilon}),
\end{aligned}
$$

such that the annulus $\mathcal{M}_{\varepsilon}$ will be at $a_{\varepsilon}=b_{\varepsilon}=0$, the local stable and unstable manifolds $W_{l o c}^{s}\left(\mathcal{M}_{\varepsilon}\right)$ and $W_{l o c}^{u}\left(\mathcal{M}_{\varepsilon}\right)$ will be at $a_{\varepsilon}=0$ and $b_{\varepsilon}=0$, respectively, and 
the stable and unstable fibers will be at $a_{\varepsilon}=0, \chi_{\varepsilon}=$ constant, $\psi_{\varepsilon}=$ constant, and $b_{\varepsilon}=0, \chi_{\varepsilon}=$ constant, $\psi_{\varepsilon}=$ constant, respectively. After dropping the subscript $\varepsilon$, these requirements, together with the invariance of the stable and unstable fiber families, imply equations (6.1).

Equations (6.1) are a special case of the Fenichel normal form, see Theorem 11.1 in Fenichel [14]. See also Tin [63].

Further simplification of equations (6.1) cannot be achieved near the whole annulus $\mathcal{M}_{\varepsilon}$. However, such simplification may be achieved locally near each part of the $N$-bump singular intersection surface $\Sigma_{0}^{\lambda}\left(\bar{\phi}_{1}, \ldots, \bar{\phi}_{N}, \bar{\theta}_{0,1}, \ldots, \bar{\theta}_{0, N}\right)$ that lies on the annulus $\mathcal{M}_{0}$ between the lines $\theta=\bar{\theta}_{0, i-1}\left(I_{0}, \lambda\right)+\Delta \theta_{+}$and $\theta=\bar{\theta}_{0, i}\left(I_{0}, \lambda\right)-\Delta \theta_{-}$ and that contains the orbit segment $O_{i}(\bar{\lambda})$. The $\chi-\psi$ coordinates on the annulus $\mathcal{M}_{\varepsilon}$ can be changed locally near each such part into $u-v$ coordinates that rectify the slow flow on $\mathcal{M}_{\varepsilon}$ so that $u^{\prime}=1$ and $v^{\prime}=0$ on $\mathcal{M}_{\varepsilon}$. When $\varepsilon=0$, we can think of $u$ as an angle and $v$ as an action variable of the outer system (3.7). Equations (6.1) in the $a-b-u-v$ coordinates become

$$
\begin{gathered}
\dot{a}=\left[A(a, b)+\sqrt{\varepsilon} F^{a}(a, b, u, v, \lambda, \sqrt{\varepsilon})\right] a, \\
\dot{b}=\left[B(a, b)+\sqrt{\varepsilon} F^{b}(a, b, u, v, \lambda, \sqrt{\varepsilon})\right] b, \\
\dot{u}=\sqrt{\varepsilon}+\left\langle a, m^{u}(a, b, u, v, \lambda, \sqrt{\varepsilon}) b\right\rangle, \\
\dot{v}=\left\langle a, m^{v}(a, b, u, v, \lambda, \sqrt{\varepsilon}) b\right\rangle,
\end{gathered}
$$

again for some matrix-valued functions $F^{a}(a, b, u, v, \lambda, \sqrt{\varepsilon}), F^{b}(a, b, u, v, \lambda, \sqrt{\varepsilon})$, $m^{u}(a, b, u, v, \lambda, \sqrt{\varepsilon})$, and $m^{v}(a, b, u, v, \lambda, \sqrt{\varepsilon})$. The functions $m^{u}(a, b, u, v, \lambda, \sqrt{\varepsilon})$ and $m^{v}(a, b, u, v, \lambda, \sqrt{\varepsilon})$ can further be written as

$$
m^{u}(a, b, u, v, \lambda, \sqrt{\varepsilon})=m_{1}^{u}(u, v)+\sqrt{\varepsilon} m_{2}^{u}(a, b, u, v, \lambda, \sqrt{\varepsilon})
$$

and

$$
m^{v}(a, b, u, v, \lambda, \sqrt{\varepsilon})=m_{1}^{v}(u, v)+\sqrt{\varepsilon} m_{2}^{v}(a, b, u, v, \lambda, \sqrt{\varepsilon}) .
$$

Gronwall-type estimates on the systems (3.6) and (3.7) and the smoothness results of Proposition 6.1 now imply that the unperturbed and the perturbed $u-v$ coordinates of any point in any compact set in which these coordinates can be defined are $\mathcal{O}(\sqrt{\varepsilon})$ close to each other.

We now define the neighborhood $U_{\delta}\left(\mathcal{M}_{\varepsilon}\right)$ of the annulus $\mathcal{M}_{\varepsilon}$ to be

$$
U_{\delta}\left(\mathcal{M}_{\varepsilon}\right)=\{(a, b, \chi, \psi) \mid\|a\|<\delta,\|b\|<\delta\} .
$$

We assume for the next proposition that the trajectory in question is entirely contained in an open subset of the neighborhood $U_{\delta}\left(\mathcal{M}_{\varepsilon}\right)$ where the flow can be rectified so that equations (6.4) are valid.

Proposition 6.3. Let $\kappa$ be any number smaller than the smallest real part of any eigenvalue of the matrix $A(0,0)$, and let $\delta$ and $\sqrt{\varepsilon}$ be small enough. Then for any trajectory that enters the neighborhood $U_{\delta}\left(\mathcal{M}_{\varepsilon}\right)$ through its face $\|b\|=\delta$ at the time $t=0$ and leaves $U_{\delta}\left(\mathcal{M}_{\varepsilon}\right)$ through its face $\|a\|=\delta$ at the time $t=T$, and any time $0<t<T$, we have the estimates:

$$
\|a(t)\| \leq C_{a} \delta e^{\kappa(t-T)},
$$




$$
\begin{gathered}
\|b(t)\| \leq C_{b} \delta e^{-\kappa t}, \\
|u(t)-\sqrt{\varepsilon} t-u(0)| \leq C_{u} \delta^{2} T e^{-\kappa T}, \\
|v(t)-v(0)| \leq C_{v} \delta^{2} T e^{-\kappa T} .
\end{gathered}
$$

Here, $C_{a}, C_{b}, C_{u}$, and $C_{v}$ are constants independent of $\delta$ and $\varepsilon$.

Proof. We first prove the estimate (6.5b); the estimate (6.5a) can be proven in the same way after inverting the time. To prove the estimate $(6.5 \mathrm{~b})$, we write equation $(6.4 \mathrm{~b})$ as

$$
\dot{b}=\left[B(0,0)+B_{a}(a, b) a+B_{b}(a, b) b+\sqrt{\varepsilon} F^{b}(a, b, u, v, \lambda, \sqrt{\varepsilon})\right] b,
$$

where $B_{a}(a, b)$ and $B_{b}(a, b)$ are tensors of rank three that depend smoothly on the variables $a$ and $b$.

Let $0<s<t<T$. If we write $b(t)=e^{B(0,0)(t-s)} \hat{b}(t)$, we obtain the equation

$$
\dot{\hat{b}}=\left[B_{a}(a, b) a+B_{b}(a, b) b+\sqrt{\varepsilon} F^{b}(a, b, u, v, \lambda, \sqrt{\varepsilon})\right] \hat{b} .
$$

Taking the norms, we obtain

$$
\|\hat{b}\| \cdot\|\dot{\hat{b}}\| \leq\left(K_{1} \delta+K_{2} \sqrt{\varepsilon}\right)\|\hat{b}\|,
$$

where the constants $K_{1}$ and $K_{2}$ are finite because the functions $B_{a}(a, b), B_{b}(a, b)$, and $F^{b}(a, b, u, v, \lambda, \sqrt{\varepsilon})$ are continuous and the set over which we are taking their suprema is compact. Hence,

$$
\|\hat{b}(t)\| \leq C_{1 b} e^{\left(K_{1} \delta+K_{2} \sqrt{\varepsilon}\right)(t-s)}\|\hat{b}(s)\|
$$

for some constant $C_{1 b}$, and

$$
\|b(t)\| \leq\left\|e^{B(0,0)(t-s)}\right\|\|\hat{b}(t)\| \leq C_{1 b} C_{2 b} e^{-\kappa_{b}(t-s)} e^{\left(K_{1} \delta+K_{2} \sqrt{\varepsilon}\right)(t-s)}\|\hat{b}(s)\|,
$$

where $C_{2 b}$ is some other constant and $\kappa_{b}$ is any number smaller than the smallest real part of any eigenvalue of the matrix $A(0,0)$, that is, $-\kappa_{b}$ is any number larger than the largest real part of any eigenvalue of the matrix $B(0,0)$.

If $\kappa$ is any number smaller than $\kappa_{b}$, then for small enough $\delta$ and $\sqrt{\varepsilon}$, we obtain the estimate

$$
\|b(t)\| \leq C_{b} e^{-\kappa(t-s)}\|\hat{b}(s)\|=C_{b} e^{-\kappa(t-s)}\|b(s)\|,
$$

where $C_{b}=C_{1 b} C_{2 b}$, since $\hat{b}(s)=b(s)$ by definition. In particular, for $s=0$ we have $\|b(s)\|=\delta$, and therefore the estimate (6.5b) follows for all $0<t<T$. The analogous estimates for the variable $a$ are

$$
\|a(t)\| \leq C_{a} e^{\kappa(t-s)}\|a(s)\|
$$

for all $0<t<s<T$, and, since $\|a(T)\|=\delta$, also the estimate (6.5a) for $0<t<T$.

We now show the proof of the estimate $(6.5 \mathrm{~d})$; the proof of $(6.5 \mathrm{c})$ is almost identical. Integrating equation $(6.4 \mathrm{~d})$ yields

$$
|v(t)-v(0)| \leq \int_{0}^{t}|\dot{v}| d s=\int_{0}^{t}\left|\left\langle a, m^{v}(a, b, u, v, \lambda, \sqrt{\varepsilon}) b\right\rangle\right| d s \leq d_{v} \int_{0}^{t}\|a\|\|b\| d s,
$$




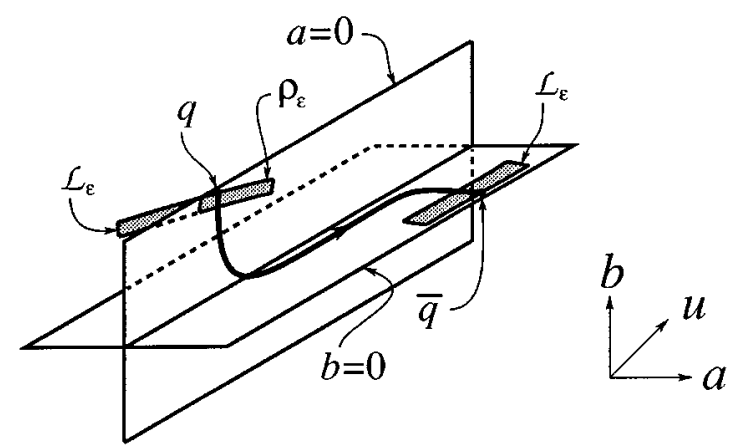

FiguRE 15. A sketch of the phase space in the neighborhood $U_{\delta}\left(\mathcal{M}_{\varepsilon}\right)$ of the normally hyperbolic slow annulus $\mathcal{M}_{\varepsilon}$ illustrating Proposition 6.5. The $v$-coordinate is suppressed.

where $d_{v}$ is a bound on the norm of the matrix $m^{v}(a, b, u, v, \lambda, \sqrt{\varepsilon})$. The integral $\int_{0}^{t}\|a\|\|b\| d s$ in (6.8) may be estimated by using the inequalities (6.5b) and (6.5a):

$$
\int_{0}^{t}\|a\|\|b\| d s \leq C_{a} C_{b} \delta^{2} \int_{0}^{t} e^{\kappa(s-T)} e^{-\kappa s} d s=C_{a} C_{b} \delta^{2} t e^{-\kappa T} \leq C_{a} C_{b} \delta^{2} T e^{-\kappa T} .
$$

Combining the estimates (6.8) and (6.9) so that $C_{v}=d_{v} C_{a} C_{b}$ concludes the proof of the estimate $(6.5 \mathrm{~d})$.

We remark that this proposition is very similar to Lemma 3.1 in Jones et al. [29].

The following proposition is a consequence of the estimate (6.5c), and is of crucial importance in ensuring that the exchange lemma with exponentially small error applies to the problem described in this paper.

Proposition 6.4. Let the positive numbers $\kappa, \delta$, and $\sqrt{\varepsilon}$ be small enough so that the assumptions of Proposition 6.3 hold, and let some trajectory enter the neighborhood $U_{\delta}\left(\mathcal{M}_{\varepsilon}\right)$ through its face $\|b\|=\delta$ at the time $t=0$ and exit $U_{\delta}\left(\mathcal{M}_{\varepsilon}\right)$ through its face $\|a\|=\delta$ at the time $t=T$. Then there exists a constant $K>0$, independent of $\delta$ and $\varepsilon$, such that if the magnitude of the difference $|u(T)-u(0)|$ of the $u$-coordinates between the exit and entrance points of that trajectory satisfies the inequality $|u(T)-u(0)|>K \delta^{2}$, then $T=\mathcal{O}(1 / \sqrt{\varepsilon})$.

Proof. Recall the estimate (6.5c). The expression on its right-hand side has a maximum at $T=1 / \kappa$, where it is equal to $C_{u} \delta^{2} / \kappa e$, so that $|u(T)-\sqrt{\varepsilon} T-u(0)|<$ $C_{u} \delta^{2} / \kappa e$. If we let $|u(T)-u(0)|>K \delta^{2}$, where $K=2 C_{u} / \kappa e$, then we must have $T=\mathcal{O}(1 / \sqrt{\varepsilon})$.

We are now ready to state the version of the exchange lemma needed in this paper. See Figure 15 for an illustration. Again, we assume that the trajectory mentioned in this lemma is entirely contained in a subregion of the neighborhood $U_{\delta}\left(\mathcal{M}_{\varepsilon}\right)$ in which the variables $a-b-u-v$ can be defined. The statement of the lemma is based on that of Tin [63]. 
Proposition 6.5. Let $\mathcal{L}_{\varepsilon}$ be an $(n+2)$-dimensional, locally invariant manifold, and let $\rho_{\varepsilon}$ be the $(n+1)$-dimensional intersection of the manifold $\mathcal{L}_{\varepsilon}$ with the face $\|b\|=\delta$ of the neighborhood $U_{\delta}\left(\mathcal{M}_{\varepsilon}\right)$. Assume that the manifolds $\rho_{\varepsilon}$ and $W_{\text {loc }}^{s}\left(\mathcal{M}_{\varepsilon}\right)$ intersect transversely, and let $q$ be a point on $\rho_{\varepsilon}$ whose trajectory exits from the neighborhood $U_{\delta}\left(\mathcal{M}_{\varepsilon}\right)$ through its face at $\|a\|=\delta$ at the point $\bar{q}$ after a time $t=\mathcal{O}(1 / \sqrt{\varepsilon})$. Then, for $\sqrt{\varepsilon}$ small, the manifold $\mathcal{L}_{\varepsilon}$ and its tangent space at the point $\bar{q}$ are $\mathcal{O}\left(e^{-\frac{c}{\sqrt{\varepsilon}}}\right)$ close to the local unstable manifold $W_{\text {loc }}^{u}\left(\mathcal{M}_{\varepsilon}\right)$ and its tangent space, respectively.

An immediate corollary to Proposition 6.5 follows from Proposition 6.4:

Proposition 6.6. The conclusion of Proposition 6.5 also holds if the assumption in it that the trajectory between the points $q$ and $\bar{q}$ must take an $\mathcal{O}(1 / \sqrt{\varepsilon})$ amount of time between these points is replaced by the assumption that the $u$ coordinates of the points $q$ and $\bar{q}$ differ by an amount larger than $K \delta^{2}$, where $K$ is the constant in Proposition 6.4.

\section{Persistence of Multi-Bump Homoclinic Intersection Surfaces}

In this section we prove Lemma 1 . We do this by piecing together the knowledge about the one-bump homoclinic intersection surfaces $\Sigma_{\varepsilon}^{\lambda}\left(\bar{\phi}_{i}, \bar{\theta}_{0, i}\right)$, with $i=$ $1, \ldots, N$, away from the annulus $\mathcal{M}_{\varepsilon}$ and the behavior of orbits on the intersecting pieces of the stable and unstable manifolds $W^{s}\left(\mathcal{M}_{\varepsilon}\right)$ and $W^{u}\left(\mathcal{M}_{\varepsilon}\right)$ as these orbits pass near $\mathcal{M}_{\varepsilon}$. The two main tools that we combine in this proof are the Poincaré-Arnold-Melnikov method and the exchange lemma, Proposition 6.5.

Before proceeding with the proof of Lemma 1, we must review the multidimensional Poincaré-Arnold-Melnikov method in slightly more detail. This review follows closely the exposition in Wiggins [66]. We will need the facts described in this review for the proof of Proposition 7.2. In particular, we must show that the $\mathcal{O}(\varepsilon)$ estimate of the distance between the stable and unstable manifolds $W^{s}\left(\mathcal{M}_{\varepsilon}\right)$ and $W^{u}\left(\mathcal{M}_{\varepsilon}\right)$, which is computed in the $x-I-\theta$ coordinates, stays of the same order as we switch to the $x-h-\theta$ coordinates.

The essence of the Poincaré-Arnold-Melnikov method consists of calculating the distance between appropriately chosen pairs of points $p_{\varepsilon}^{s}$ and $p_{\varepsilon}^{u}$, with $p_{\varepsilon}^{s}$ lying on the stable manifold $W^{s}\left(\mathcal{M}_{\varepsilon}\right)$ and $p_{\varepsilon}^{u}$ lying on the unstable manifold $W^{u}\left(\mathcal{M}_{\varepsilon}\right)$, which are found in the following way. We first use the unperturbed homoclinic manifold $W(\mathcal{M})$ as the framework for parametrizing the manifolds $W^{s}\left(\mathcal{M}_{\varepsilon}\right)$ and $W^{u}\left(\mathcal{M}_{\varepsilon}\right)$. At every point $p$ on the homoclinic manifold $W(\mathcal{M})$ we consider the space $\mathcal{N}_{p} W(\mathcal{M})$, spanned by the vectors $D_{x} K_{i}(p), i=1, \ldots, n$. Inside any small enough neighborhood of the point $p$, the space $\mathcal{N}_{p} W(\mathcal{M})$ intersects the homoclinic manifold $W(\mathcal{M})$ transversely in precisely the point $p$. Therefore, $\mathcal{N}_{p} W(\mathcal{M})$ also intersects the perturbed manifolds $W^{s}\left(\mathcal{M}_{\varepsilon}\right)$ and $W^{u}\left(\mathcal{M}_{\varepsilon}\right)$ transversely in discrete points, $\mathcal{O}(\varepsilon)$ away from the point $p$. Now, since the manifolds $W^{s}\left(\mathcal{M}_{\varepsilon}\right)$ and $W^{u}\left(\mathcal{M}_{\varepsilon}\right)$ may fold back onto themselves, they may intersect the transverse space $\mathcal{N}_{p} W(\mathcal{M})$ in more than one point each. However, the unique choice of two points, $p_{\varepsilon}^{s}$ in $W^{s}\left(\mathcal{M}_{\varepsilon}\right)$ and $p_{\varepsilon}^{u}$ in $W^{u}\left(\mathcal{M}_{\varepsilon}\right)$, which is consistent with the perturbation theory is the following. The point $p_{\varepsilon}^{s}$ is the unique intersection point of the space $\mathcal{N}_{p} W(\mathcal{M})$ and the stable manifold $W^{s}\left(\mathcal{M}_{\varepsilon}\right)$ inside an $\mathcal{O}(\varepsilon)$ neighborhood of $p$, whose trajectory takes the least amount of time to reach the local stable manifold $W_{\text {loc }}^{s}\left(\mathcal{M}_{\varepsilon}\right)$ of the annulus $\mathcal{M}_{\varepsilon}$. We choose the point $p_{\varepsilon}^{u}$ in an analogous manner.

Now that we have properly identified the points $p_{\varepsilon}^{s}$ and $p_{\varepsilon}^{u}$, we can state 
Proposition 7.1. In the $x-I-\theta$ coordinates, the signed length of the projection of the vector $p_{\varepsilon}^{u}-p_{\varepsilon}^{s}$ onto the vector $D_{x} K_{i}(p)$ is equal to

$$
d_{i}(p, \varepsilon)=\frac{\varepsilon M_{i}\left(I, \phi, \theta_{0}, \lambda\right)}{\left\|D_{x} K_{i}(p)\right\|}+\mathcal{O}\left(\varepsilon^{2}\right),
$$

where the integral representing the $i$-th component $M_{i}\left(I, \phi, \theta_{0}, \lambda\right)$ of the Melnikov vector, given by formula (3.1), is evaluated along the trajectory passing through the point $p$.

The proof of this proposition is standard. It can be found, for instance, in Wiggins [66].

Now, in the $x-h-\theta$ coordinates, a point $p$ on the manifold $W(\mathcal{M})$ is given by an appropriate choice of the variables $t, \phi$, and $\theta_{0}$ in the formulas (2.6) with $I=I_{0}+\sqrt{\varepsilon} h$. Therefore, we can write

$$
d_{i}(p, \varepsilon)=\frac{\varepsilon M_{i}\left(I_{0}, \phi, \theta_{0}, \lambda\right)}{\left\|D_{x} K_{i}\left(p_{0}\right)\right\|}+\mathcal{O}(\varepsilon \sqrt{\varepsilon})
$$

where $p_{0}$ is the point given by (2.6) with the same values of $t, \phi$, and $\theta_{0}$ as the point $p$, and $I=I_{0}$. In other words, the distance between the stable and unstable manifolds $W^{s}\left(\mathcal{M}_{\varepsilon}\right)$ and $W^{u}\left(\mathcal{M}_{\varepsilon}\right)$ is of the size $\mathcal{O}(\varepsilon)$ also in the $x-h-\theta$ coordinates.

Next, we need to elaborate on some geometric details of the singular intersection surface $\Sigma_{0}^{\lambda}\left(\bar{\phi}_{1}, \ldots, \bar{\phi}_{N}, \bar{\theta}_{0,1}, \ldots, \bar{\theta}_{0, N}\right)$. In particular, the $h$ coordinates of the intersections between this surface and the lines $\theta=\bar{\theta}_{0, i}\left(I_{0}, \lambda\right)+\Delta \theta_{+}$and $\theta=\bar{\theta}_{0, i}\left(I_{0}, \lambda\right)-\Delta \theta_{-}$are contained in the intervals $h_{i, \min }^{+}<h<h_{i, \max }^{+}$and $h_{i, \text { min }}^{-}<h<h_{i, \max }^{-}$, respectively. Since orbits of the outer system (3.7) have vertical tangents at $h=0$, it follows from Assumption 6 that the $h$ coordinate along each of these intervals is of the same sign on the whole interval. From the definition of the singular intersection surface $\Sigma_{0}^{\lambda}\left(\bar{\phi}_{1}, \ldots, \bar{\phi}_{N}, \bar{\theta}_{0,1}, \ldots, \bar{\theta}_{0, N}\right)$ following Assumption 6, we can infer that the intervals $h_{i, \min }^{ \pm}<h<h_{i, \max }^{ \pm}$are either all finite or all semi-infinite. In the latter case the $h$ coordinate has the same sign on all of these intervals, and we restrict the surface $\Sigma_{0}^{\lambda}\left(\bar{\phi}_{1}, \ldots, \bar{\phi}_{N}, \bar{\theta}_{0,1}, \ldots, \bar{\theta}_{0, N}\right)$ to be compact, so that all of the numbers $h_{i, \min }^{ \pm}$and $h_{i, \max }^{ \pm}$become finite. We choose this restriction in such a way that the part of the boundary of the redefined $\Sigma_{0}^{\lambda}\left(\bar{\phi}_{1}, \ldots, \bar{\phi}_{N}, \bar{\theta}_{0,1}, \ldots, \bar{\theta}_{0, N}\right)$ along which an infinite piece of the original $\Sigma_{0}^{\lambda}\left(\bar{\phi}_{1}, \ldots, \bar{\phi}_{N}, \bar{\theta}_{0,1}, \ldots, \bar{\theta}_{0, N}\right)$ is cut off consists of subsequent heteroclinic orbits of the inner system and orbit segments of the outer system, see Figure 6 in $\S 4$.

Under the coordinate change from the $x-h-\theta$ to the $a-b-u-v$ coordinates introduced in the previous section, the line segments

$$
\left\{(h, \theta) \mid \theta=\bar{\theta}_{0, i}\left(I_{0}, \lambda\right)+\Delta \theta_{+}, h_{i, \min }^{+}<h<h_{i, \max }^{+}\right\}
$$

with $i=1, \ldots, N-1$ are mapped onto curves parametrized by the functions $u=u_{i}^{+}(v)$, defined on some intervals $v_{i, \min }^{+}<v<v_{i, \max }^{+}$. Likewise, the line segments

$$
\left\{(h, \theta) \mid \theta=\bar{\theta}_{0, i}\left(I_{0}, \lambda\right)-\Delta \theta_{-}, h_{i, \min }^{-}<h<h_{i, \max }^{-}\right\}
$$

with $i=2, \ldots, N$ are mapped onto curves parametrized by the functions $u_{i}^{-}(v)$ defined for $v_{i, \min }^{-}<v<v_{i, \max }^{-}$. These parametrizations are possible because orbits of the outer system (3.7) intersect the original line segments transversely by Assumption 6. Notice that $v_{i, \min }^{+}=v_{i+1, \min }^{-}$and $v_{i, \max }^{+}=v_{i+1, \max }^{-}$. 


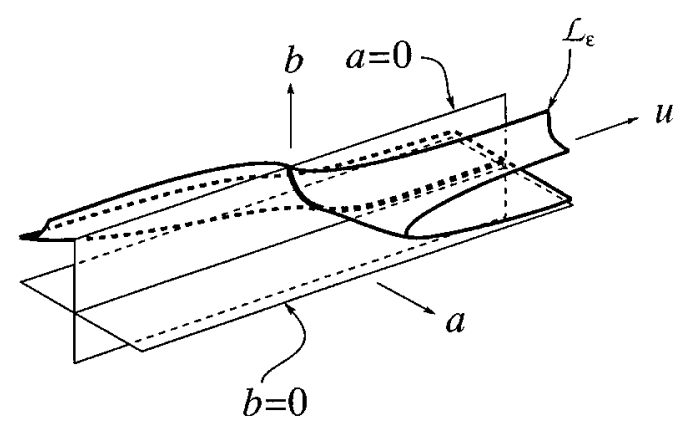

FiguRE 16. A sketch of the shape of the manifold $\mathcal{L}_{\varepsilon}$ as it intersects the stable manifold $W^{s}\left(\mathcal{M}_{\varepsilon}\right)$, that is the hyperplane $a=0$, inside the neighborhood $U_{\delta}\left(\mathcal{M}_{\varepsilon}\right)$. Orbits which are not close enough to the intersection surface drift out of the neighborhood $U_{\delta}\left(\mathcal{M}_{\varepsilon}\right)$ after times shorter than $\mathcal{O}(1 / \sqrt{\varepsilon})$.

Finally, for $i=2, \ldots, N-1$ and any small positive $\eta$ and $\delta$, we let $U_{\eta, \delta}^{ \pm}(i)$ denote the set

$$
\begin{gathered}
U_{\eta, \delta}^{ \pm}(i)=\left\{(a, b, u, v)|| u-u_{i}^{ \pm}(v) \mid<\eta,\|a\|<\delta,\|b\|<\delta,\right. \\
\left.v_{i, \text { min }}^{ \pm}-\delta<v<v_{i, \max }^{ \pm}+\delta\right\} .
\end{gathered}
$$

The sets $U_{\eta, \delta}^{+}(1)$ and $U_{\eta, \delta}^{-}(N)$ are defined in the same way. The sets $U_{\eta, \delta}^{-}(1)$ and $U_{\eta, \delta}^{+}(N)$ are defined in a similar manner, but in the $a-b-\chi-\psi$ coordinates instead of the $a-b-u-v$ coordinates, which need not be defined near the lines $\theta=\bar{\theta}_{0,1}\left(I_{0}, \lambda\right)-\Delta \theta_{-}$and $\theta=\bar{\theta}_{0, N}\left(I_{0}, \lambda\right)+\Delta \theta_{+}$.

We can now prove Lemma 1 in two major steps. The first step describes the existence of trajectories that satisfy the hypotheses of the exchange lemma, Proposition 6.5. The second step involves a sequence of propositions that constitute the actual proof of Lemma 1.

The first step is covered in the next proposition. Note that the perturbed $a-$ $b-u-v$ coordinates are used throughout this proof.

Proposition 7.2. Let $\delta$ be small enough so that $K \delta^{2} \ll \delta$, where $K$ is the constant given in Proposition 6.4. Let $\mathcal{L}_{\varepsilon}$ be any smooth, $(n+2)$-dimensional, locally invariant manifold, which is $\mathcal{O}\left(e^{-\frac{c}{\sqrt{\varepsilon}}}\right)$ close to the unstable manifold $W^{u}\left(\mathcal{M}_{\varepsilon}\right)$ in a neighborhood of where this manifold intersects the stable manifold $W^{s}\left(\mathcal{M}_{\varepsilon}\right)$ along the single-bump intersection surface $\Sigma_{\varepsilon}^{\lambda}\left(\bar{\phi}_{i}, \bar{\theta}_{0, i}\right)$, outside of the neighborhood $U_{\delta}\left(\mathcal{M}_{\varepsilon}\right)$. Let the tangent spaces of the manifolds $\mathcal{L}_{\varepsilon}$ and $W^{u}\left(\mathcal{M}_{\varepsilon}\right)$ also be $\mathcal{O}\left(e^{-\frac{c}{\sqrt{\varepsilon}}}\right)$ close. Then near every point $p$ on the local unstable manifold $W_{\text {loc }}^{u}\left(\mathcal{M}_{\varepsilon}\right)$ (that is, on $\|b\|=0)$ with $\|a\|=\delta$, whose $u$ and $v$ coordinates are contained in the strip $u_{i}^{+}(v)+\delta<u<u_{i+1}^{-}(v)+\delta, v_{i, \min }^{+}+\delta<v<v_{i, \max }^{+}-\delta$, there exists a point $\bar{q}$ on the manifold $\mathcal{L}_{\varepsilon}$ whose $a, u$, and $v$ coordinates are the same as those of the point $p$, and whose $b$ coordinate is of the size $\mathcal{O}\left(e^{-\frac{c}{\sqrt{\varepsilon}}}\right)$. The tangent space of the manifold $\mathcal{L}_{\varepsilon}$ at the point $\bar{q}$ is also $\mathcal{O}\left(e^{-\frac{c}{\sqrt{\varepsilon}}}\right)$ close to the tangent space of the local unstable manifold $W_{\text {loc }}^{u}\left(\mathcal{M}_{\varepsilon}\right)$.

See Figure 16 for an illustration. 
Proof. Let $\rho_{\varepsilon}$ be the intersection of the manifold $\mathcal{L}_{\varepsilon}$ with the face $\|b\|=\delta$ of the neighborhood $U_{\delta}\left(\mathcal{M}_{\varepsilon}\right)$. The manifolds $\mathcal{L}_{\varepsilon}, W^{u}\left(\mathcal{M}_{\varepsilon}\right)$ and $W^{s}\left(\mathcal{M}_{\varepsilon}\right)$ are all spanned by orbits of equations (6.4), and the face $\|b\|=\delta$ intersects these orbits transversely at an $\mathcal{O}(1)$-size angle for all small enough $a$. Moreover, the manifold $W^{u}\left(\mathcal{M}_{\varepsilon}\right)$, and therefore also the manifold $\mathcal{L}_{\varepsilon}$, intersect the local stable manifold $W_{l o c}^{s}\left(\mathcal{M}_{\varepsilon}\right)$ transversely at an $\mathcal{O}(\varepsilon)$-size angle near $\|b\|=\delta$ along two intersection surfaces that are also spanned by orbits. Hence these two intersection surfaces also intersect the face $\|b\|=\delta$ transversely at an $\mathcal{O}(1)$-size angle. Therefore, the manifolds $\rho_{\varepsilon}$ and $W_{\text {loc }}^{s}\left(\mathcal{M}_{\varepsilon}\right)$ intersect transversely at an $\mathcal{O}(\varepsilon)$-size angle, so that $\rho_{\varepsilon}$ satisfies the assumptions of the exchange lemma, Proposition 6.5.

Because the size of the angle between the manifolds $\rho_{\varepsilon}$ and $W_{l o c}^{s}\left(\mathcal{M}_{\varepsilon}\right)$ is only $\mathcal{O}(\varepsilon)$, it is not immediately clear that the trajectory through any point on $\rho_{\varepsilon}$ should exit the neighborhood $U_{\delta}\left(\mathcal{M}_{\varepsilon}\right)$ at a point whose $u$ and $v$ coordinates are contained in the strip $u_{i}^{+}(v)+\delta<u<u_{i+1}^{-}(v)+\delta, v_{i, \min }^{+}+\delta<v<v_{i, \max }^{+}-\delta$. We now show, however, that this is the case and, in particular, that there exist points on the manifold $\rho_{\varepsilon}$ that get mapped even closer to $u=u_{i}^{+}(v)$ than $u=u_{i}^{+}(v)+\delta$.

We proceed as follows. First, Gronwall-type estimates show that the surfaces $\Sigma_{\varepsilon}^{\lambda}\left(\bar{\phi}_{i}, \bar{\theta}_{0, i}\right)$ and $\Sigma_{0}^{\lambda}\left(\bar{\phi}_{i}, \bar{\theta}_{0, i}\right)$ are $\mathcal{O}(\sqrt{\varepsilon})$ close outside the neighborhood $U_{\delta}\left(\mathcal{M}_{\varepsilon}\right)$. This and the exponential closeness of the manifolds $\mathcal{L}_{\varepsilon}$ and $W^{u}\left(\mathcal{M}_{\varepsilon}\right)$ imply that the intersection curve $C_{\varepsilon}$ of the manifold $\rho_{\varepsilon}$ with the local stable manifold $W_{\text {loc }}^{s}\left(\mathcal{M}_{\varepsilon}\right)$ (that is, the hyperplane $a=0$ ) is contained within an $\mathcal{O}(\sqrt{\varepsilon})$ neighborhood of the curve $C_{0}$ along which the surface $\Sigma_{0}^{\lambda}\left(\bar{\phi}_{i}, \bar{\theta}_{0, i}\right)$ intersects the face $\|b\|=\delta$. But the (perturbed) $u-v$ coordinates along the curve $C_{0}$ are $\mathcal{O}(\sqrt{\varepsilon})$ close to $u=u_{i}^{+}(v)$, $v_{i, \min }^{+}<v<v_{i, \max }^{+}$, so that those of the curve $C_{\varepsilon}$ are too.

Let $\hat{\rho}_{\varepsilon}$ be the part of the intersection manifold $\rho_{\varepsilon}$ that lies within a neighborhood of the curve $C_{\varepsilon}$ of size $\frac{1}{4} \delta$. Let $\partial \hat{\rho}_{\varepsilon}$ be the boundary of $\hat{\rho}_{\varepsilon}$. Let $r$ be any point in the boundary $\partial \hat{\rho}_{\varepsilon}$, and let it have the $u-v$ coordinates $\left(u_{r}, v_{r}\right)$, with $v_{i, \min }^{+}+\delta<$ $v_{r}<v_{i, \max }^{+}-\delta$. As implied by Proposition 7.1, and the discussion following it, in particular formula (7.1), there exists some positive constant $L$ independent of the point $r$, so that we have the estimate $\|a(0)\| \geq L \delta \varepsilon$ for the norm of the $a$ coordinate of the point $r$.

Let $T$ be the time when the trajectory that starts at the point $r$ at $t=0$ reaches the face $\|a\|=\delta$ of the neighborhood $U_{\delta}\left(\mathcal{M}_{\varepsilon}\right)$. Then the further estimate

$$
\|a(t)\| \geq \frac{\|a(0)\| e^{\kappa t}}{C_{a}} \geq \frac{L \delta \varepsilon e^{\kappa t}}{C_{a}}
$$

for some $\kappa>0$ that follows from equation (6.7) implies that for $t=T$, we have $\delta \geq \frac{L \delta \varepsilon e^{\kappa T}}{C_{a}}$. Hence

$$
T \leq \frac{1}{\kappa} \ln \frac{C_{a}}{L \varepsilon}
$$

Therefore, the fact that the $u-v$ coordinates along the curve $C_{\varepsilon}$ are $\mathcal{O}(\sqrt{\varepsilon})$ close to $u=u_{i}^{+}(v), v_{i, \min }^{+}<v<v_{i, \max }^{+}$and Proposition 6.4 show that for sufficiently small $\varepsilon$, the point $\bar{r}$, where the trajectory through the point $r$ exits the neighborhood $U_{\delta}\left(\mathcal{M}_{\varepsilon}\right)$, has its $u$-coordinate $u_{\bar{r}}$ less than $u_{i}^{+}\left(v_{r}\right)+\frac{1}{2} \delta$.

Now, the $v$ coordinates $v_{r}$ and $v_{\bar{r}}$ of the points $r$ and $\bar{r}$ are not the same, so what we really have to show is the inequality $u_{\bar{r}}<u_{i}^{+}\left(v_{\bar{r}}\right)+\delta$. However, this inequality 
is true because the estimates (7.2) and (6.5d) imply the inequality

$$
\left|v_{\bar{r}}-v_{r}\right| \leq \frac{C_{v} L \delta^{2} \varepsilon}{C_{a} k} \ln \frac{C_{a}}{L \varepsilon} .
$$

We thus immediately conclude that

$$
\left|u_{i}^{+}\left(v_{\bar{r}}\right)-u_{i}^{+}\left(v_{r}\right)\right| \leq E_{i} \frac{C_{v} L \delta^{2} \varepsilon}{C_{a} k} \ln \frac{C_{a}}{L \varepsilon}=\mathcal{O}\left(\delta^{2} \varepsilon \ln \frac{1}{\varepsilon}\right),
$$

where

$$
E_{i}=\max \left\{\left|\frac{d u_{i}^{+}}{d v}(v)\right| \mid v_{i, \min }^{+}<v<v_{i, \max }^{+}\right\} .
$$

The rest of the proof now proceeds in exactly the same way as the proof of Lemma 4.1 in Jones and Kopell [28], and therefore we omit it.

We remark that this proposition plays a similar role in the proof of our Lemma 1 as Lemma 4.1 in Jones and Kopell [28] plays in the proof of their Theorem 4.1.

We now turn to the proof of Lemma 1. The proof will consist of several parts, each of which will be listed as a separate proposition.

Proposition 7.3. The homoclinic intersection surface

$$
\Sigma_{\varepsilon}^{\lambda}\left(\bar{\phi}_{1}, \ldots, \bar{\phi}_{N}, \bar{\theta}_{0,1}, \ldots, \bar{\theta}_{0, N}\right)
$$

exists.

Proof. Let $\lambda$ be near $\bar{\lambda}$, so that the singular intersection surface

$$
\Sigma_{0}^{\lambda}\left(\bar{\phi}_{1}, \ldots, \bar{\phi}_{N}, \bar{\theta}_{0,1}, \ldots, \bar{\theta}_{0, N}\right)
$$

exists. Consider the intersection of the local unstable manifold $W_{l o c}^{u}\left(\mathcal{M}_{\varepsilon}\right)$ with the open neighborhood $U_{\delta, \delta}^{-}(1)$. Extend this intersection by evolving initial conditions contained in it under the forward-time flow of the equations (2.1) to a portion of the global unstable manifold $W^{u}\left(\mathcal{M}_{\varepsilon}\right)$. We denote this portion by $\mathcal{L}_{\varepsilon}$.

By Assumption 5 and Proposition 3.1, the manifold $\mathcal{L}_{\varepsilon}$ intersects the stable manifold $W^{s}\left(\mathcal{M}_{\varepsilon}\right)$ transversely along the single-bump intersection surface $\Sigma_{\varepsilon}^{\lambda}\left(\bar{\phi}_{1}, \bar{\theta}_{0,1}\right)$. Thus the hypotheses of Proposition 7.2 are satisfied for the manifold $\mathcal{L}_{\varepsilon}$ near the surface $\Sigma_{\varepsilon}^{\lambda}\left(\bar{\phi}_{1}, \bar{\theta}_{0,1}\right)$ upon its entry into the neighborhood $U_{\delta, \delta}^{+}(1)$. We can conclude that, at the points where $\mathcal{L}_{\varepsilon}$ leaves the neighborhood $U_{\delta}\left(\mathcal{M}_{\varepsilon}\right)$ of the annulus $\mathcal{M}_{\varepsilon}$ inside the neighborhood $U_{\delta, \delta}^{-}(2)$, the manifold $\mathcal{L}_{\varepsilon}$ and its tangent space are $\mathcal{O}\left(e^{-\frac{c}{\sqrt{\varepsilon}}}\right)$ close to the local unstable manifold $W_{\text {loc }}^{u}\left(\mathcal{M}_{\varepsilon}\right)$ and its tangent space, respectively. Since the manifold $W_{\text {loc }}^{u}\left(\mathcal{M}_{\varepsilon}\right)$ intersects the stable manifold $W^{s}\left(\mathcal{M}_{\varepsilon}\right)$ transversely at an angle of size $\mathcal{O}(\varepsilon)$ along a piece of the single bump intersection surface $\Sigma_{\varepsilon}^{\lambda}\left(\bar{\phi}_{2}, \bar{\theta}_{0,2}\right)$, it follows that the manifold $\mathcal{L}_{\varepsilon}$ must also intersect the stable manifold $W^{s}\left(\mathcal{M}_{\varepsilon}\right)$ transversely as they leave the neighborhood $U_{\delta, \delta}^{-}(2)$. The $\mathcal{O}\left(e^{-\frac{c}{\sqrt{\varepsilon}}}\right)$ closeness estimate for the manifolds $W^{u}\left(\mathcal{M}_{\varepsilon}\right)$ and $\mathcal{L}_{\varepsilon}$ and their tangent spaces can be extended to points in the vicinity of the whole single-bump intersection surface $\Sigma_{\varepsilon}^{\lambda}\left(\bar{\phi}_{2}, \bar{\theta}_{0,2}\right)$. Therefore, the hypotheses of Proposition 7.2 are again satisfied for the manifold $\mathcal{L}_{\varepsilon}$ upon its entry into the neighborhood $U_{\delta, \delta}^{+}(2)$.

The argument can now proceed by induction on $i$, the consecutive number of the bump, which shows that the manifold $\mathcal{L}_{\varepsilon}$ intersects the stable manifold $W^{s}\left(\mathcal{M}_{\varepsilon}\right)$ transversely near the single-bump intersection surface $\Sigma_{\varepsilon}^{\lambda}\left(\bar{\phi}_{i}, \bar{\theta}_{0, i}\right)$ for each $i \geq 3$, and therefore also near $\Sigma_{\varepsilon}^{\lambda}\left(\bar{\phi}, \bar{\theta}_{0, N}\right)$. Following the points on this last intersection 
backwards in time under the flow of the system (2.1) (or the equivalent system (3.3)) until they reach the local unstable manifold $W_{l o c}^{u}\left(\mathcal{M}_{\varepsilon}\right)$ inside the neighborhood $U_{\delta, \delta}^{-}(1)$, we obtain the desired intersection surface $\Sigma_{\varepsilon}^{\lambda}\left(\bar{\phi}_{1}, \ldots, \bar{\phi}_{N}, \bar{\theta}_{0,1}, \ldots, \bar{\theta}_{0, N}\right)$.

The $N$-bump homoclinic intersection surface $\Sigma_{0}^{\lambda}\left(\bar{\phi}_{1}, \ldots, \bar{\phi}_{N}, \bar{\theta}_{0,1}, \ldots, \bar{\theta}_{0, N}\right)$ has $N$ pieces outside of the neighborhood $U_{\delta}\left(\mathcal{M}_{\varepsilon}\right)$ of the annulus $\mathcal{M}_{\varepsilon}$. In particular, let the piece that is outside $U_{\delta}\left(\mathcal{M}_{\varepsilon}\right)$ during the $i$-th excursion be denoted by $\Sigma_{\varepsilon}^{\lambda}(i)$. Then, we have

Proposition 7.4. Outside the neighborhood $U_{\delta}\left(\mathcal{M}_{\varepsilon}\right)$, the surface $\Sigma_{\varepsilon}^{\lambda}(i)$ is $\mathcal{O}(\sqrt{\varepsilon})$ close to the limiting single-bump homoclinic intersection surface $\Sigma_{0}^{\lambda}\left(\bar{\phi}_{i}, \bar{\theta}_{0, i}\right)$.

The proof of this proposition follows by standard Gronwall-type estimates from

Proposition 7.5. Outside the neighborhood $U_{\delta}\left(\mathcal{M}_{\varepsilon}\right)$, the surface $\Sigma_{\varepsilon}^{\lambda}(i)$ is $\mathcal{O}\left(e^{-\frac{c}{\sqrt{\varepsilon}}}\right)$ close to the single-bump homoclinic intersection surface $\Sigma_{\varepsilon}^{\lambda}\left(\bar{\phi}_{i}, \bar{\theta}_{0, i}\right)$.

Proof. On the face $\|a\|=\delta$ of the neighborhood $U_{\delta, \delta}^{-}(i)$, the manifolds $\mathcal{L}_{\varepsilon}$ and $W_{\text {loc }}^{u}\left(\mathcal{M}_{\varepsilon}\right)$ are $\mathcal{O}\left(e^{-\frac{c}{\sqrt{\varepsilon}}}\right)$ close by the exchange lemma. Therefore the manifolds $\mathcal{L}_{\varepsilon}$ and $W^{u}\left(\mathcal{M}_{\varepsilon}\right)$ must be $\mathcal{O}\left(e^{-\frac{c}{\sqrt{\varepsilon}}}\right)$ close along the whole $i$-th bump by Gronwall-type estimates.

We now invert the time, and denote by $\mathcal{K}_{\varepsilon}$ the portion of the manifold $W_{\text {loc }}^{s}\left(\mathcal{M}_{\varepsilon}\right)$ inside the neighborhood $U_{\delta, \delta}^{+}(N)$, analogous to $\mathcal{L}_{\varepsilon}$. The whole construction of the intersection surface $\Sigma_{\varepsilon}^{\lambda}\left(\bar{\phi}_{1}, \ldots, \bar{\phi}_{N}, \bar{\theta}_{0,1}, \ldots, \bar{\theta}_{0, N}\right)$ goes through as before, and it is clear that this surface is contained in the intersection of the manifolds $\mathcal{L}_{\varepsilon}$ and $\mathcal{K}_{\varepsilon}$. We immediately see that on the face $\|b\|=\delta$ of the neighborhood $U_{\delta, \delta}^{+}(i)$, the manifolds $\mathcal{K}_{\varepsilon}$ and $W_{\text {loc }}^{s}\left(\mathcal{M}_{\varepsilon}\right)$ must be $\mathcal{O}\left(e^{-\frac{c}{\sqrt{\varepsilon}}}\right)$ close, and as above, the manifolds $\mathcal{K}_{\varepsilon}$ and $W^{u}\left(\mathcal{M}_{\varepsilon}\right)$ must be $\mathcal{O}\left(e^{-\frac{c}{\sqrt{\varepsilon}}}\right)$ close along the whole $i$-th bump by Gronwall-type estimates.

Therefore, since the manifolds $W^{s}\left(\mathcal{M}_{\varepsilon}\right)$ and $W^{u}\left(\mathcal{M}_{\varepsilon}\right)$ intersect at an angle of size $\mathcal{O}(\varepsilon)$ along the single-bump intersection surface $\Sigma_{\varepsilon}^{\lambda}\left(\bar{\phi}_{i}, \bar{\theta}_{0, i}\right)$, the intersection $\Sigma_{\varepsilon}^{\lambda}(i)$ of the manifolds $\mathcal{L}_{\varepsilon}$ and $\mathcal{K}_{\varepsilon}$ may be at most a distance $\mathcal{O}\left(e^{-\frac{c}{\sqrt{\varepsilon}}} / \varepsilon\right)$ away. Taking a slightly smaller constant $c$, the estimate becomes $\mathcal{O}\left(e^{-\frac{c}{\sqrt{\varepsilon}}}\right)$.

Proposition 7.4 took care of the parts of $\Sigma_{\varepsilon}^{\lambda}\left(\bar{\phi}_{1}, \ldots, \bar{\phi}_{N}, \bar{\theta}_{0,1}, \ldots, \bar{\theta}_{0, N}\right)$ that were outside the neighborhood $U_{\delta}\left(\mathcal{M}_{\varepsilon}\right)$. We now have to take care of the portions of $\Sigma_{\varepsilon}^{\lambda}\left(\bar{\phi}_{1}, \ldots, \bar{\phi}_{N}, \bar{\theta}_{0,1}, \ldots, \bar{\theta}_{0, N}\right)$ that stay near the annulus $\mathcal{M}_{\varepsilon}$. We denote the $i$-th such portion by $\mathcal{M}_{\varepsilon}^{\lambda}(i)$. Then we have

Proposition 7.6. Outside the neighborhoods $U_{\delta, \delta}^{+}(i-1)$ and $U_{\delta, \delta}^{-}(i)$, the surfaces $\mathcal{M}_{\varepsilon}^{\lambda}(i)$ and $\mathcal{M}_{0}$ are $\mathcal{O}(\sqrt{\varepsilon})$ close.

This proposition is an immediate consequence of the fact that the annuli $\mathcal{M}_{\varepsilon}$ and $\mathcal{M}_{0}$ are $\mathcal{O}(\sqrt{\varepsilon})$ close, and of the next proposition:

Proposition 7.7. Outside the neighborhoods $U_{\delta, \delta}^{+}(i-1)$ and $U_{\delta, \delta}^{-}(i)$, the surfaces $\mathcal{M}_{\varepsilon}^{\lambda}(i)$ and $\mathcal{M}_{\varepsilon}$ are $\mathcal{O}\left(e^{-\frac{c}{\sqrt{\varepsilon}}}\right)$ close.

Proof. This follows because the norms $\|a\|$ and $\|b\|$ are $\mathcal{O}\left(e^{-\frac{c}{\sqrt{\varepsilon}}}\right)$ small there by Proposition 6.4 and estimates (6.5a) and (6.5b). 
Proof of Lemma 1. Propositions 7.3, 7.4, and 7.7 provide the existence of the $N$ bump intersection surface $\Sigma_{\varepsilon}^{\lambda}\left(\bar{\phi}_{1}, \ldots, \bar{\phi}_{N}, \bar{\theta}_{0,1}, \ldots, \bar{\theta}_{0, N}\right)$ and its closeness to its singular counterpart

$$
\Sigma_{0}^{\lambda}\left(\bar{\phi}_{1}, \ldots, \bar{\phi}_{N}, \bar{\theta}_{0,1}, \ldots, \bar{\theta}_{0, N}\right)
$$

away from the corners of $\Sigma_{0}^{\lambda}\left(\bar{\phi}_{1}, \ldots, \bar{\phi}_{N}, \bar{\theta}_{0,1}, \ldots, \bar{\theta}_{0, N}\right)$ at $\theta=\bar{\theta}_{0, i}\left(I_{0}, \lambda\right)-\Delta \theta_{-}$ and $\theta=\bar{\theta}_{0, i}\left(I_{0}, \lambda\right)+\Delta \theta_{+}$. The closeness of the two manifolds near the corners is provided by the observation that both Kaplun's and Eckhaus' extension theorems (see Lagerstrom [41], Eckhaus [10], respectively) make it possible to take $\delta$ to be a function $\delta=\delta(\varepsilon)$ such that $\delta(\varepsilon) \rightarrow 0$ as $\varepsilon \rightarrow 0$ in Propositions 7.4-7.7. The transversality of the intersection follows from the fact that, near their intersections along each of the single bump surfaces $\Sigma_{\varepsilon}^{\lambda}\left(\bar{\phi}_{i}, \bar{\theta}_{0, i}\right)$, the manifolds $\mathcal{K}_{\varepsilon}$ and $\mathcal{L}_{\varepsilon}$ and their tangent spaces are $\mathcal{O}\left(e^{-\frac{c}{\sqrt{\varepsilon}}}\right)$ close to the manifolds $W^{s}\left(\mathcal{M}_{\varepsilon}\right)$ and $W^{u}\left(\mathcal{M}_{\varepsilon}\right)$ and their tangent spaces, respectively.

\section{Closeness Estimates for Orbits on HOMOCLINIC INTERSECTION SURFACES}

In this section, we prove the closeness estimate for orbits on the $N$-bump intersection surface $\Sigma_{\varepsilon}^{\lambda}\left(\bar{\phi}_{1}, \ldots, \bar{\phi}_{N}, \bar{\theta}_{0,1}, \ldots, \bar{\theta}_{0, N}\right)$ and singular orbits on the $N$-bump singular intersection surface $\Sigma_{0}^{\lambda}\left(\bar{\phi}_{1}, \ldots, \bar{\phi}_{N}, \bar{\theta}_{0,1}, \ldots, \bar{\theta}_{0, N}\right)$ that was stated in Lemma 2 and illustrated in Figure 12. We will see that this estimate depends crucially on Lemma 1, and in particular on the estimates stated in Propositions 7.4-7.7. We caution that this closeness estimate need not be valid for orbits that do not lie on the surface $\Sigma_{\varepsilon}^{\lambda}\left(\bar{\phi}_{1}, \ldots, \bar{\phi}_{N}, \bar{\theta}_{0,1}, \ldots, \bar{\theta}_{0, N}\right)$.

Proof of Lemma 2. Throughout this proof, all orbits, perturbed and unperturbed, are studied using the perturbed $a-b-u-v$ coordinate system. We begin with the first bump. We trace two trajectories passing through the points $p_{\varepsilon}$ and $p_{0}$ at $t=0$. By the usual Gronwall-type estimate, these two trajectories are a distance $\mathcal{O}(\sqrt{\varepsilon})$ apart for all finite times $t$. In particular, we let $t=T$ be large enough so that the slower of the two trajectories reaches the $\|b\|=\delta$ face of the neighborhood $U_{\delta}\left(\mathcal{M}_{\varepsilon}\right)$.

Let $q_{\varepsilon}$ and $q_{0}$ denote the points on the curves $\mathcal{A}_{\varepsilon}$ and $\mathcal{A}_{0}$ at $t=T$. By what was stated in the previous paragraph, the points $q_{\varepsilon}$ and $q_{0}$ are $\mathcal{O}(\sqrt{\varepsilon})$ close to each other. We now show that the points $r_{\varepsilon}$ and $r_{0}$, where the curves $\mathcal{A}_{\varepsilon}$ and $\mathcal{A}_{0}$, respectively, exit the neighborhood $U_{\delta, \delta}^{-}(2)$ through its boundary $\|a\|=\delta$, are still $\mathcal{O}(\sqrt{\varepsilon})$ close as follows.

The (perturbed) $u-v$ coordinates of the point $\hat{q}_{0}$ where the curve $\mathcal{A}_{0}$ lands on the unperturbed annulus $\mathcal{M}_{0}$ are at most $\mathcal{O}(\sqrt{\varepsilon})$ away from the $u-v$ coordinates of the point $q_{0}$. (The unperturbed $u-v$ coordinates of these two points are identical.) We now consider two trajectories: the trajectory that passes through the point $q_{\varepsilon}$ and lies on the orbit $\mathcal{A}_{\varepsilon}$ together with the unperturbed slow-time trajectory that passes through the point $\hat{q}_{0}$ and lies on the singular orbit $\mathcal{A}_{0}$. In particular, we monitor the $v$ coordinates of these trajectories under the slow-time, $\tau=\sqrt{\varepsilon}$, evolution, after they both have passed through the two respective initial points $q_{\varepsilon}$ and $\hat{q}_{0}$ at $\tau=0$. By Proposition 6.4 and estimate (6.5d), the $v$ coordinate of the trajectory through the point $q_{\varepsilon}$ stays within an $\mathcal{O}\left(e^{-\frac{c}{\sqrt{\varepsilon}}}\right)$ distance from the $v$ coordinate of the point $q_{\varepsilon}$, and therefore at most an $\mathcal{O}(\sqrt{\varepsilon})$ distance away from the $v$ coordinate of the point $\hat{q}_{0}$ for as long as this trajectory stays in the neighborhood 


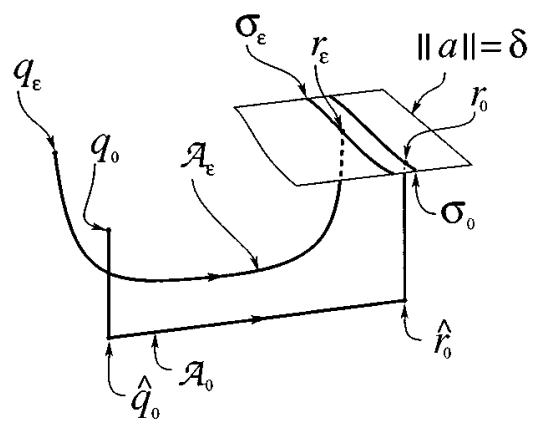

Figure 17. An illustration of the proof of Lemma 2. Since the $u-v$ coordinates along the orbits $\mathcal{A}_{\varepsilon}$ and $\mathcal{A}_{0}$ are $\mathcal{O}(\sqrt{\varepsilon})$ close, and the lines $\sigma_{\varepsilon}$ and $\sigma_{0}$ are also $\mathcal{O}(\sqrt{\varepsilon})$ close, then so must be the points $r_{\varepsilon}$ and $r_{0}$.

$U_{\delta}\left(\mathcal{M}_{\varepsilon}\right)$. Equations (6.4c) and $(6.4 \mathrm{~d})$ at $a=b=0$ rewritten in terms of the slow time $\tau=\sqrt{\varepsilon} t$ read $u^{\prime}=1, v^{\prime}=0$. These equations, and the fact that the perturbed $u-v$ coordinates of any point are $\mathcal{O}(\sqrt{\varepsilon})$ close to their unperturbed counterparts, imply that the (perturbed) $v$ coordinate of the unperturbed trajectory through the point $\hat{q}_{0}$ differs from that of $\hat{q}_{0}$ by an $\mathcal{O}(\sqrt{\varepsilon})$ amount, for as long as the $u-v$ coordinates are well defined near this trajectory.

Now consider the points $r_{\varepsilon}$ and $r_{0}$ where the curves $\mathcal{A}_{\varepsilon}$ and $\mathcal{A}_{0}$ pierce the boundary $\|a\|=\delta$ of the neighborhood $U_{\delta, \delta}^{-}(2)$. Since the (perturbed) $u-v$ coordinates of the point $r_{0}$ are at most $\mathcal{O}(\sqrt{\varepsilon})$ away from those of the point $\hat{r}_{0}$, at which the curve $\mathcal{A}_{0}$ takes off from the annulus $\mathcal{M}_{0}$, it follows from the discussion of the preceding paragraph that the $v$ coordinates of the points $r_{\varepsilon}$ and $r_{0}$ must be at most $\mathcal{O}(\sqrt{\varepsilon})$ apart. But by Proposition 7.4, the curves $\sigma_{\varepsilon}$ and $\sigma_{0}$, along which the surfaces $\Sigma_{\varepsilon}^{\lambda}\left(\bar{\phi}_{1}, \ldots, \bar{\phi}_{N}, \bar{\theta}_{0,1}, \ldots, \bar{\theta}_{0, N}\right)$ and $\Sigma_{0}^{\lambda}\left(\bar{\phi}_{1}, \ldots, \bar{\phi}_{N}, \bar{\theta}_{0,1}, \ldots, \bar{\theta}_{0, N}\right)$ intersect the face $\|a\|=\delta$ of the neighborhood $U_{\delta, \delta}^{-}(2)$, and on which the points $r_{\varepsilon}$ and $r_{0}$ lie, are also at most $\mathcal{O}(\sqrt{\varepsilon})$ apart from each other. Moreover, the $u-v$ coordinates along both these curves are $\mathcal{O}(\sqrt{\varepsilon})$ close to $u=u_{2}^{-}(v), v_{2, \min }^{-}<v<v_{2, \max }^{-}$. (The $b$ coordinates along them are also at most of the size $\mathcal{O}(\sqrt{\varepsilon})$.) Hence, the points $r_{\varepsilon}$ and $r_{0}$ can be at most $\mathcal{O}(\sqrt{\varepsilon})$ apart, see Figure 17 .

It remains to prove that the segments of the curves $\mathcal{A}_{\varepsilon}$ and $\mathcal{A}_{0}$ that are inside the neighborhood $U_{\delta}\left(\mathcal{M}_{\varepsilon}\right)$ and between the neighborhoods $U_{\delta, \delta}^{+}(1)$ and $U_{\delta, \delta}^{-}(2)$ are $\mathcal{O}(\sqrt{\varepsilon})$ apart. The proof goes as follows. As above, we recall Proposition 6.4 and estimates $(6.5 \mathrm{c})$ and $(6.5 \mathrm{~d})$, and the fact that the $u-v$ coordinates change at most by an $\mathcal{O}(\sqrt{\varepsilon})$ amount between the perturbed and unperturbed systems. We again rewrite equations (6.4c) and (6.4d) at $a=b=0$ in terms of the slow time $\tau=\sqrt{\varepsilon} t$. We then conclude that the $u-v$ coordinates of the two perturbed and unperturbed slow-time trajectories through the points $q_{\varepsilon}$ and $\hat{q}_{0}$, respectively, remain at most a distance $\mathcal{O}(\sqrt{\varepsilon})$ apart for as long as the trajectory on the curve $\mathcal{A}_{\varepsilon}$ stays within the neighborhood $U_{\delta}\left(\mathcal{M}_{\varepsilon}\right)$. Moreover, by Proposition 6.4 and estimates (6.5a) and (6.5b), the $a-b$ coordinates of this trajectory are of the size $\mathcal{O}\left(e^{-\frac{c}{\sqrt{\varepsilon}}}\right)$ inside the neighborhood $U_{\delta}\left(\mathcal{M}_{\varepsilon}\right)$ between the neighborhoods $U_{\delta, \delta}^{+}(1)$ and $U_{\delta, \delta}^{-}(2)$, and the $a-b$ coordinates of the unperturbed slow-time trajectory on the curve $\mathcal{A}_{0}$ are of the size 
$\mathcal{O}(\sqrt{\varepsilon})$ because the annuli $\mathcal{M}_{\varepsilon}$ and $\mathcal{M}_{0}$ are at most $\mathcal{O}(\sqrt{\varepsilon})$ apart. Therefore, the segments of the curves $\mathcal{A}_{\varepsilon}$ and $\mathcal{A}_{0}$ that are inside the neighborhood $U_{\delta}\left(\mathcal{M}_{\varepsilon}\right)$ and between the neighborhoods $U_{\delta, \delta}^{+}(1)$ and $U_{\delta, \delta}^{-}(2)$ are indeed at most a distance $\mathcal{O}(\sqrt{\varepsilon})$ apart.

The proof is now completed by finite induction on $i$, the consecutive number of the bump. The difference between the neighborhoods $U_{\delta}\left(\mathcal{M}_{\varepsilon}\right)$ used in this proof and $V_{\delta}$ used in the statement of Lemma 2 is insignificant: Since the transformation between the $x-h-\theta$ and $a-b-\chi-\psi$ coordinates is smooth and hence continuous, one can easily see that for all small enough $\varepsilon$, every $\delta$-sized neighborhood of any compact subannulus of the annulus $\mathcal{M}_{\varepsilon}$ in the $x-h-\theta$ coordinates contains a slightly smaller $\delta$-sized neighborhood of a corresponding subannulus of the annulus $\mathcal{M}_{0}$ in the $a-b-\chi-\psi$ coordinates, and vice versa. Finally, the statement about the function $\delta(\varepsilon)$ is shown in the same way as in the proof of Lemma 1.

\section{Persistence and Closeness Estimates for Modified Multi-Bump HOMOCLINIC INTERSECTION SURFACES}

In this section, we prove Lemmas 3 and 4 . Their proofs are modified versions of the proofs of Lemmas 1 and 2. The modifications are needed in order to cover the case of modified $N$-bump singular homoclinic intersection surfaces. We will not state these proofs in their entirety, but only highlight the new aspects.

First, we recall from Assumption 7 that the piece of the modified $N$-bump singular transition orbit $\Gamma$ between the points $c_{2}(\bar{\lambda})$ and $d_{N+1}(\bar{\lambda})$ satisfies Assumption 6 . Thus, this piece, say $\Gamma_{2, N+1}$, is an ordinary $(N-1)$-bump singular transition orbit, and implies the existence of an ordinary $(N-1)$-bump singular intersection surface, which contains the singular orbit $\Gamma_{2, N+1}$ in its interior. We restrict this singular intersection surface slightly, so that at the end point on the line $\theta=\bar{\theta}_{0,2}\left(I_{0}, \bar{\lambda}\right)-\Delta \theta_{-}$ of each $(N-1)$-bump singular transition orbit composing this smaller surface, the line $\theta=\bar{\theta}_{0,2}\left(I_{0}, \bar{\lambda}\right)-\Delta \theta_{-}$and the orbit of the outer system (3.7) that passes through that end point intersect transversely on the annulus $\mathcal{M}_{0}$. We denote this restricted $(N-1)$-bump singular intersection surface by $\Sigma_{0}^{\bar{\lambda}}\left(\bar{\phi}_{2}, \ldots, \bar{\phi}_{N}, \bar{\theta}_{0,2}, \ldots, \bar{\theta}_{0, N}\right)$.

Note that the surface $\Sigma_{0}^{\bar{\lambda}}\left(\bar{\phi}_{2}, \ldots, \bar{\phi}_{N}, \bar{\theta}_{0,2}, \ldots, \bar{\theta}_{0, N}\right)$ still contains the singular transition orbit $\Gamma_{2, N+1}$ in its interior, and also contains the portion of the original modified $N$-bump singular intersection surface $\hat{\Sigma}_{0}^{\bar{\lambda}}\left(\bar{\phi}_{1}, \ldots, \bar{\phi}_{N}, \bar{\theta}_{0,1}, \ldots, \bar{\theta}_{0, N}\right)$ that stretches from the second bump to the $N$-th bump. Furthermore, if we evolve in backward time under the dynamics of the outer system (3.7) all the trajectories of the points on the segment along which the line $\theta=\bar{\theta}_{0,2}\left(I_{0}, \bar{\lambda}\right)-\Delta \theta_{-}$and the surface $\Sigma_{0}^{\bar{\lambda}}\left(\bar{\phi}_{2}, \ldots, \bar{\phi}_{N}, \bar{\theta}_{0,2}, \ldots, \bar{\theta}_{0, N}\right)$ intersect, the region on the annulus $\mathcal{M}_{0}$ swept out by these trajectories will contain in its interior the segment along which the line $\theta=\bar{\theta}_{0,1}\left(I_{0}, \bar{\lambda}\right)+\Delta \theta_{+}$and the surface $\hat{\Sigma}_{0}^{\bar{\lambda}}\left(\bar{\phi}_{1}, \ldots, \bar{\phi}_{N}, \bar{\theta}_{0,1}, \ldots, \bar{\theta}_{0, N}\right)$ intersect.

The above situation will persist for all $\lambda$ close enough to $\lambda=\bar{\lambda}$. We can now define the neighborhoods $U_{\eta, \delta}^{ \pm}(i)$ with $i=2, \ldots, N$ corresponding to the $(N-1)$ bump singular intersection surface $\Sigma_{0}^{\lambda}\left(\bar{\phi}_{2}, \ldots, \bar{\phi}_{N}, \bar{\theta}_{0,2}, \ldots, \bar{\theta}_{0, N}\right)$ in the same way as in section 5 , except that we can now use the $a-b-u-v$ coordinates to define the neighborhood $U_{\eta, \delta}^{-}(2)$. We define the neighborhoods $U_{\eta, \delta}^{ \pm}(1)$ in the $a-b-\chi-\psi$ coordinates.

First, we state the analog of Proposition 7.3. 
Proposition 9.1. The homoclinic intersection surface

$$
\hat{\Sigma}_{\varepsilon}^{\lambda}\left(\bar{\phi}_{1}, \ldots, \bar{\phi}_{N}, \bar{\theta}_{0,1}, \ldots, \bar{\theta}_{0, N}\right)
$$

exists. The stable and unstable manifolds $W^{s}\left(\mathcal{M}_{\varepsilon}\right)$ and $W^{u}\left(\mathcal{M}_{\varepsilon}\right)$ intersect transversely along this two-dimensional surface.

Proof. Consider the intersection of the local stable manifold $W_{l o c}^{s}\left(\mathcal{M}_{\varepsilon}\right)$ of the annulus $\mathcal{M}_{\varepsilon}$ with the neighborhood $U_{\delta, \delta}^{+}(N)$. Extend this manifold backward in time, and call the resulting manifold $\mathcal{K}_{\varepsilon}$. Likewise, define the manifold $\mathcal{L}_{\varepsilon}$ by extending forward in time the intersection of the local unstable manifold $W_{l o c}^{u}\left(\mathcal{M}_{\varepsilon}\right)$ with the neighborhood $U_{\delta, \delta}^{-}(1)$.

Following the manifold $\mathcal{K}_{\varepsilon}$ in backward time, we can show as in the proof of Lemma 1 that there exist $(N+1-i)$-bump homoclinic intersection surfaces that connect neighborhoods $U_{\delta, \delta}^{-}(i)$ to the neighborhood $U_{\delta, \delta}^{+}(N)$ for each $i=2, \ldots, N$. We continue the manifold $\mathcal{K}_{\varepsilon}$ using Proposition 7.2 inside the neighborhood $U_{\delta}\left(\mathcal{M}_{\varepsilon}\right)$ to the neighborhood $U_{\delta, \delta}^{+}(1)$. On the face $\|b\|=\delta$ of this neighborhood, the manifold $\mathcal{K}_{\varepsilon}$ is $\mathcal{O}\left(e^{-\frac{c}{\sqrt{\varepsilon}}}\right)$ close to the local stable manifold $W_{\text {loc }}^{s}\left(\mathcal{M}_{\varepsilon}\right)$, and the corresponding tangent spaces are also $\mathcal{O}\left(e^{-\frac{c}{\sqrt{\varepsilon}}}\right)$ close. Now, from Proposition 3.1 it follows that the manifold $W_{l o c}^{s}\left(\mathcal{M}_{\varepsilon}\right)$ intersects the manifold $\mathcal{L}_{\varepsilon}$ transversely near the face $\|b\|=\delta$ of this neighborhood along a piece of the single-bump homoclinic intersection surface $\Sigma_{\varepsilon}^{\lambda}\left(\bar{\phi}_{1}, \bar{\theta}_{0,1}\right)$. Therefore, the manifold $\mathcal{K}_{\varepsilon}$ must intersect the manifold $\mathcal{L}_{\varepsilon}$ transversely along a surface $\hat{\Sigma}_{\varepsilon}^{\lambda}\left(\bar{\phi}_{1}, \ldots, \bar{\phi}_{N}, \bar{\theta}_{0,1}, \ldots, \bar{\theta}_{0, N}\right)$, which is locally $\mathcal{O}\left(e^{-\frac{c}{\sqrt{\varepsilon}}}\right)$ close to the surface $\Sigma_{\varepsilon}^{\lambda}\left(\bar{\phi}_{1}, \bar{\theta}_{0,1}\right)$. Since the time flow of system (2.1) is a diffeomorphism, and diffeomorphisms preserve transverse intersections, the manifolds $\mathcal{K}_{\varepsilon}$ and $\mathcal{L}_{\varepsilon}$ must intersect transversely everywhere along the surface $\hat{\Sigma}_{\varepsilon}^{\lambda}\left(\bar{\phi}_{1}, \ldots, \bar{\phi}_{N}, \bar{\theta}_{0,1}, \ldots, \bar{\theta}_{0, N}\right)$.

We next state the analog of Proposition 7.5. We denote the piece of the homoclinic intersection surface $\hat{\Sigma}_{\varepsilon}^{\lambda}\left(\bar{\phi}_{1}, \ldots, \bar{\phi}_{N}, \bar{\theta}_{0,1}, \ldots, \bar{\theta}_{0, N}\right)$ that is outside the neighborhood $U_{\delta}\left(\mathcal{M}_{\varepsilon}\right)$ during the $i$-th excursion by $\hat{\Sigma}_{\varepsilon}^{\lambda}(i)$. Then, we have the following:

Proposition 9.2. Outside the neighborhood $U_{\delta}\left(\mathcal{M}_{\varepsilon}\right)$, every point on the surface $\hat{\Sigma}_{\varepsilon}^{\lambda}(i)$ is $\mathcal{O}\left(e^{-\frac{c}{\sqrt{\varepsilon}}}\right)$ close to some point on the single-bump homoclinic intersection surface $\Sigma_{\varepsilon}^{\lambda}\left(\bar{\phi}_{i}, \bar{\theta}_{0, i}\right)$.

Proof. The proof of the previous proposition yields the conclusion of this proposition for $i=1$.

For $i \geq 2$, we recall that near the face $\|b\|=\delta$ of the neighborhood $U_{\delta, \delta}^{+}(i)$, the manifold $\mathcal{K}_{\varepsilon}$ and the local stable manifold $W_{\text {loc }}^{s}\left(\mathcal{M}_{\varepsilon}\right)$ are $\mathcal{O}\left(e^{-\frac{c}{\sqrt{\varepsilon}}}\right)$ close, so that the manifold $\mathcal{K}_{\varepsilon}$ is $\mathcal{O}\left(e^{-\frac{c}{\sqrt{\varepsilon}}}\right)$ close to the stable manifold $W^{s}\left(\mathcal{M}_{\varepsilon}\right)$ along the whole $i$-th bump of the surface $\hat{\Sigma}_{\varepsilon}^{\lambda}\left(\bar{\phi}_{1}, \ldots, \bar{\phi}_{N}, \bar{\theta}_{0,1}, \ldots, \bar{\theta}_{0, N}\right)$, and in particular upon leaving the neighborhood $U_{\delta, \delta}^{-}(i)$ through its face $\|a\|=\delta$.

On the other hand, near the face $\|b\|=\delta$ of the neighborhood $U_{\delta, \delta}^{+}(i-1)$, the manifold $\mathcal{K}_{\varepsilon}$ is again $\mathcal{O}\left(e^{-\frac{c}{\sqrt{\varepsilon}}}\right)$ close to the local stable manifold $W_{\text {loc }}^{s}\left(\mathcal{M}_{\varepsilon}\right)$, that is, the hyperplane $\|a\|=0$. Therefore, we must have by inequality (6.7) that the time of flight of all trajectories on the surface $\hat{\Sigma}_{\varepsilon}^{\lambda}\left(\bar{\phi}_{1}, \ldots, \bar{\phi}_{N}, \bar{\theta}_{0,1}, \ldots, \bar{\theta}_{0, N}\right)$ inside the neighborhood $U_{\delta}\left(\mathcal{M}_{\varepsilon}\right)$ between the face $\|b\|=\delta$ of the neighborhood $U_{\delta, \delta}^{+}(i-1)$ and the face $\|a\|=\delta$ of the neighborhood $U_{\delta, \delta}^{-}(i)$ is $\mathcal{O}(1 / \sqrt{\varepsilon})$. By estimate $(6.5 \mathrm{~b})$, 
it follows that all orbits on $\hat{\Sigma}_{\varepsilon}^{\lambda}\left(\bar{\phi}_{1}, \ldots, \bar{\phi}_{N}, \bar{\theta}_{0,1}, \ldots, \bar{\theta}_{0, N}\right)$ pass through the face $\|a\|=\delta$ of the neighborhood $U_{\delta, \delta}^{-}(i)$ at most $\mathcal{O}\left(e^{-\frac{c}{\sqrt{\varepsilon}}}\right)$ away from where the local unstable manifold $W_{l o c}^{u}\left(\mathcal{M}_{\varepsilon}\right)$, that is the hyperplane $\|b\|=0$, does.

We can therefore conclude that all orbits on $\hat{\Sigma}_{\varepsilon}^{\lambda}\left(\bar{\phi}_{1}, \ldots, \bar{\phi}_{N}, \bar{\theta}_{0,1}, \ldots, \bar{\theta}_{0, N}\right)$ pass through the face $\|a\|=\delta$ of the neighborhood $U_{\delta, \delta}^{-}(i)$ at most an $\mathcal{O}\left(e^{-\frac{c}{\sqrt{\varepsilon}}}\right)$-size distance away from where the intersection of the manifolds $W_{\text {loc }}^{u}\left(\mathcal{M}_{\varepsilon}\right)$ and $W^{s}\left(\mathcal{M}_{\varepsilon}\right)$ along the surface $\Sigma_{\varepsilon}^{\lambda}\left(\bar{\phi}_{i}, \bar{\theta}_{0, i}\right)$ does, possibly for a slightly smaller constant $c$. The conclusion of the proposition follows by Gronwall-type estimates.

We remark that this proof also works for Proposition 7.5, but the proof presented there is simpler.

The proof of Lemma 3 now follows from the above two propositions, Propositions 7.4, 7.6 and 7.7, and Kaplun's and Eckhaus' extension theorems.

Finally, we describe the

Proof of Lemma 4. We define the manifolds $\mathcal{K}_{\varepsilon}$ and $\mathcal{L}_{\varepsilon}$ as in the proof of lemma 3 . For any $\eta>0$ independent of $\varepsilon$, the two portions of the modified $N$-bump intersection surface $\hat{\Sigma}_{\varepsilon}^{\lambda}\left(\bar{\phi}_{1}, \ldots, \bar{\phi}_{N}, \bar{\theta}_{0,1}, \ldots, \bar{\theta}_{0, N}\right)$ spanned by trajectories that start at $t=0$ with $h>\eta$ or $h<\eta$ inside the neighborhood $U_{\delta, \delta}^{-}(1)$ are each an ordinary $N$-bump intersection surface. So by Lemmas 1 and 2, these two portions limit onto the respective portions of the modified $N$-bump singular intersection surface $\hat{\Sigma}_{0}^{\lambda}\left(\bar{\phi}_{1}, \ldots, \bar{\phi}_{N}, \bar{\theta}_{0,1}, \ldots, \bar{\theta}_{0, N}\right)$. The two limiting portions are again just two ordinary $N$-bump singular intersection surfaces. By the extension theorems of Kaplun and Eckhaus (Lagerstrom [41], Eckhaus [10]), $\eta$ can be taken to be a function of $\varepsilon$, such that $\eta(\varepsilon) \rightarrow 0$ as $\varepsilon \rightarrow 0$. Thus, the estimates of Lemma 2 hold for all orbits on the surfaces $\hat{\Sigma}_{\varepsilon}^{\lambda}\left(\bar{\phi}_{1}, \ldots, \bar{\phi}_{N}, \bar{\theta}_{0,1}, \ldots, \bar{\theta}_{0, N}\right)$ and $\hat{\Sigma}_{0}^{\lambda}\left(\bar{\phi}_{1}, \ldots, \bar{\phi}_{N}, \bar{\theta}_{0,1}, \ldots, \bar{\theta}_{0, N}\right)$ except for those whose trajectories start at $t=0$ inside the neighborhood $U_{\delta, \delta}^{-}(1)$ with their $h$ coordinates satisfying $|h|<\eta(\varepsilon)$.

In order to stretch the closeness estimate to these exceptional orbits as well, we proceed as follows. We can, for simplicity and with no loss of generality, assume that the function $\eta(\varepsilon)$ decays to zero with $\varepsilon$ more slowly than the function $\sqrt{\varepsilon}$. We consider two orbits on the surface $\hat{\Sigma}_{\varepsilon}^{\lambda}\left(\bar{\phi}_{1}, \ldots, \bar{\phi}_{N}, \bar{\theta}_{0,1}, \ldots, \bar{\theta}_{0, N}\right)$ whose trajectories start at $t=0$ inside the neighborhood $U_{\delta, \delta}^{-}(1)$ a distance, say, $2 \eta(\varepsilon)$ apart, one with $|h|>\eta(\varepsilon)$ and the other with $|h| \leq \eta(\varepsilon)$. Proposition 9.2 and an argument similar to that used in the proof of Lemma 2 show that upon their entry into the neighborhood $U_{\delta, \delta}^{-}(N)$ through its face at $\|b\|=\delta$, these two orbits are still at most a distance $\mathcal{O}(\eta(\varepsilon))$ apart.

An application of Lemma 2, and Kaplun's and Eckhaus' extension theorems conclude the proof.

\section{Multi-Bump Orbits Homoclinic to a Resonance Band}

In this section, we use Lemmas 1, 2, 3, and 4 as building blocks to establish the existence of multi-bump homoclinic and heteroclinic orbits stated in Theorems 1 through 4 . For Theorems 1, 3, and 4, the first building block is the $N$-bump homoclinic intersection surface $\Sigma_{\varepsilon}^{\lambda}\left(\bar{\phi}_{1}, \ldots, \bar{\phi}_{N}, \bar{\theta}_{0,1}, \ldots, \bar{\theta}_{0, N}\right)$, which was constructed in the course of proving Lemma 1 . The second building block for these theorems is the closeness estimate developed in Lemma 2, which ensures that orbits on a true $N$-bump homoclinic intersection surface stay close to singular $N$-bump transition 
orbits. For Theorem 2, the building blocks are the modified $N$-bump homoclinic intersection surface $\hat{\Sigma}_{\varepsilon}^{\lambda}\left(\bar{\phi}_{1}, \ldots, \bar{\phi}_{N}, \bar{\theta}_{0,1}, \ldots, \bar{\theta}_{0, N}\right)$ constructed in the proof of Lemma 3 and the closeness estimate of Lemma 4 , respectively.

We first prove Theorem 1. We prove this theorem below for the case when both $O_{1, \varepsilon}(\lambda)$ and $O_{N+1, \varepsilon}(\lambda)$ are periodic orbits. The proof for the case when the segment $O_{1, \varepsilon}(\lambda)$ is a piece of the (restricted) unstable manifold of a saddle $s_{1, \varepsilon}(\lambda)$ or when the segment $O_{N+1, \varepsilon}(\lambda)$ is a piece of the (restricted) stable manifold of a saddle $s_{N+1, \varepsilon}(\lambda)$ on $\mathcal{M}_{\varepsilon}$ follows along the same lines except for minor modifications, which are discussed in Kovačič [35].

The proof of Theorem 1 is based on the following

Proposition 10.1. For all $\lambda$ near $\lambda=\bar{\lambda}$, and all small enough positive $\varepsilon$, there exists an orbit, $\mathcal{A}_{1, \varepsilon}(\lambda)$, that is contained in both the unstable manifold $W^{u}\left(O_{1, \varepsilon}(\lambda)\right)$ and the intersection surface $\Sigma_{\varepsilon}^{\lambda}\left(\bar{\phi}_{1}, \ldots, \bar{\phi}_{N}, \bar{\theta}_{0,1}, \ldots, \bar{\theta}_{0, N}\right)$. Likewise, there exists an orbit, $\mathcal{A}_{N+1, \varepsilon}(\lambda)$, that is contained in both the stable manifold $W^{s}\left(O_{N+1, \varepsilon}(\lambda)\right)$ and the intersection surface $\Sigma_{\varepsilon}^{\lambda}\left(\bar{\phi}_{1}, \ldots, \bar{\phi}_{N}, \bar{\theta}_{0,1}, \ldots, \bar{\theta}_{0, N}\right)$. Moreover, there exists a function $\delta(\varepsilon)$ such that $\delta(\varepsilon) \rightarrow 0$ as $\varepsilon \rightarrow 0$ and the orbit $\mathcal{A}_{1, \varepsilon}(\lambda)$ is $\mathcal{O}(\delta(\varepsilon)$ ) close to the singular transition orbit $\mathcal{A}_{1,0}(\lambda)$, while the orbit $\mathcal{A}_{N+1, \varepsilon}(\lambda)$ is $\mathcal{O}(\delta(\varepsilon)$ ) close to the singular transition orbit $\mathcal{A}_{N+1,0}(\lambda)$.

Proof. We only sketch this proof briefly; the missing details are similar to those for the one-bump case found in Kovačič [35]. Furthermore, we only prove the first part of this proposition, since the second part is nearly identical.

To begin with, it easily follows from Proposition 6.2 that the local unstable manifold $W_{l o c}^{u}\left(O_{1, \varepsilon}(\lambda)\right)$ is composed of all the unstable fibers with base points on the orbit $O_{1, \varepsilon}(\lambda)$, and that this manifold limits as $\varepsilon \rightarrow 0$ onto the union of the unstable manifolds of all the equilibria on the limiting closed curve $O_{1,0}(\lambda)$ under the flow of the inner equations (3.4). This union is clearly the local unstable manifold $W_{l o c}^{u}\left(O_{1,0}(\lambda)\right)$ of the curve $O_{1,0}(\lambda)$.

Now, for $\varepsilon=0$, and for all $\lambda$ near $\bar{\lambda}$, the local unstable manifold $W_{l o c}^{u}\left(O_{1,0}(\lambda)\right)$ of the curve $O_{1,0}(\lambda)$ intersects the singular homoclinic intersection surface

$$
\Sigma_{0}^{\lambda}\left(\bar{\phi}_{1}, \ldots, \bar{\phi}_{N}, \bar{\theta}_{0,1}, \ldots, \bar{\theta}_{0, N}\right)
$$

transversely inside the unstable manifold $W_{l o c}^{u}\left(\mathcal{M}_{0}\right)$. This readily implies that for small positive $\varepsilon$ the local unstable manifold $W_{l o c}^{u}\left(O_{1, \varepsilon}(\lambda)\right)$ and the $N$-bump homoclinic intersection surface $\Sigma_{\varepsilon}^{\lambda}\left(\bar{\phi}_{1}, \ldots, \bar{\phi}_{N}, \bar{\theta}_{0,1}, \ldots, \bar{\theta}_{0, N}\right)$ intersect transversely inside the unstable manifold $W^{u}\left(\mathcal{M}_{\varepsilon}\right)$ along an orbit segment. By extending this orbit segment in forward and backward time, we obtain the desired orbit, $\mathcal{A}_{1, \varepsilon}(\lambda)$. The proximity statement follows from Lemma 2.

Proof of Theorem 1. Proposition 10.1 implies the existence of two special orbits on the homoclinic intersection surface $\Sigma_{\varepsilon}^{\lambda}\left(\bar{\phi}_{1}, \ldots, \bar{\phi}_{N}, \bar{\theta}_{0,1}, \ldots, \bar{\theta}_{0, N}\right)$, the orbit $\mathcal{A}_{1, \varepsilon}(\lambda)$ backward asymptotic to the periodic orbit $O_{1, \varepsilon}(\lambda)$ and the orbit $\mathcal{A}_{N+1, \varepsilon}(\lambda)$ forward asymptotic to the periodic orbit $O_{N+1, \varepsilon}(\lambda)$. Also, by Proposition 10.1 and because of the transversality condition for their unperturbed counterparts, $\mathcal{A}_{1,0}(\lambda)$ and $\mathcal{A}_{N+1,0}(\lambda)$, the two perturbed orbits, $\mathcal{A}_{1, \varepsilon}(\lambda)$ and $\mathcal{A}_{N+1, \varepsilon}(\lambda)$, must pass through each other at some $\lambda=\lambda(\varepsilon)$ near $\lambda=\bar{\lambda}$ with $\lambda(0)=\bar{\lambda}$ and thus form a heteroclinic orbit connecting the periodic orbit $O_{1, \varepsilon}(\lambda(\varepsilon))$ to the periodic orbit $O_{N+1, \varepsilon}(\lambda(\varepsilon))$, as claimed. 
Proof of Theorem 2. The local unstable manifolds of the points $c_{1, \varepsilon}(\lambda)$ and $c_{1,0}(\lambda)$ are $\mathcal{O}(\sqrt{\varepsilon})$ close by Proposition 6.2, since they are precisely the perturbed and unperturbed unstable fibers emanating from these two points, respectively. For some $\lambda=\lambda(\varepsilon)$ near $\lambda=\bar{\lambda}$, the unstable manifold $W^{u}\left(c_{1, \varepsilon}(\lambda)\right)$ of the point $c_{1, \varepsilon}(\lambda)$ passes transversely through the $N$-bump modified homoclinic intersection surface $\hat{\Sigma}_{\varepsilon}^{\lambda}\left(\bar{\phi}_{1}, \ldots, \bar{\phi}_{N}, \bar{\theta}_{0,1}, \ldots, \bar{\theta}_{0, N}\right)$, intersecting it along an orbit. This is because the point $c_{1,0}(\lambda)$ passes transversely through the line $\bar{\theta}_{0,1}\left(I_{0}, \lambda\right)+\Delta \theta_{-}$at $\lambda=\bar{\lambda}$. As a consequence of Lemma 4 , the unstable manifold $W^{u}\left(c_{1, \varepsilon}(\lambda(\varepsilon))\right)$ reenters the neighborhood $V_{\delta}$ of the annulus $\mathcal{M}_{\varepsilon}$ through its side $\partial V_{\delta}$ near the point $d_{N+1}(\bar{\lambda})$ a distance $\mathcal{O}(\delta(\varepsilon))$ away from where the singular transition orbit $\Gamma$ does. A simple argument using the stable fibers in Proposition 6.2 then shows that the trajectories on this unstable manifold must be attracted to the same object as the trajectories in the region $\mathcal{B}_{\varepsilon}$.

Proof of Theorem 3. The existence of the heteroclinic orbit follows from Proposition 10.1 and an argument similar to that given in the second part of the proof of Theorem 2. The transversality of the intersection is shown in the same way as the transversality for the analogous one-bump orbits is shown in Kovačič [35].

Proof of Theorem 4. The existence part of the theorem is a combination of Proposition 10.1 and the conservation of energy along orbits. The transversality part is similar to that given in Kovačič [36] for one-bump orbits.

\section{An EXAmple}

In this section, we illustrate the main results of this paper on a four-parameter family of problems in which a Duffing oscillator is coupled to an anharmonic oscillator, described by the system of equations

$$
\begin{gathered}
\dot{p}=\mu^{2} q\left(I-q^{2}\right)-\varepsilon \alpha p, \\
\dot{q}=p, \\
\dot{I}=-\varepsilon I \sin \theta-\varepsilon \beta I-\varepsilon \gamma p^{2}, \\
\dot{\theta}=I-1-\frac{1}{2} \mu^{2} q^{2}-\varepsilon \cos \theta,
\end{gathered}
$$

where $\mu, \alpha, \beta$, and $\gamma$ are positive parameters, and $\varepsilon \ll 1$ a small positive parameter. This system was introduced by Kovačič [33, 34], and is of the form (2.1) for nonzero $\alpha, \beta$ and $\gamma$, and also of the form (2.2) with the Hamiltonian

$$
\begin{aligned}
\hat{H}(p, q, I, \theta) & =H(p, q, I)+\varepsilon H_{1}(p, q, I, \theta) \\
& =\frac{1}{2} I^{2}-I+\frac{1}{2} p^{2}-\frac{1}{2} \mu^{2} q^{2}\left(I-\frac{1}{2} q^{2}\right)-\varepsilon I \cos \theta,
\end{aligned}
$$

when $\alpha=\beta=\gamma=0$. For the system (11.1) with both Hamiltonian and dissipative perturbations, the existence of several different types of single bump transition solutions has been shown in Kovačič [33, 34, 35, 36]. In this section, we use Theorems 1-4 to demonstrate the existence of multi-bump homoclinic orbits in both cases.

As is shown in Kovačič [34], one can easily verify that Assumptions 1-4 are satisfied for this system. In particular, for zero $\varepsilon$, an unstable invariant annulus, 


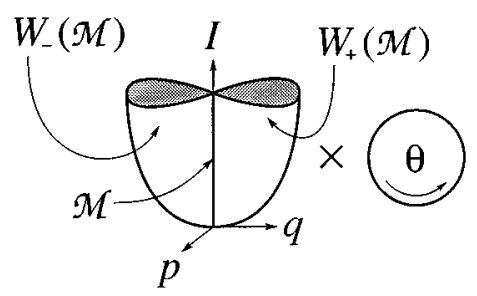

Figure 18. The annulus $\mathcal{M}$ and the homoclinic manifolds $\mathcal{W}_{+}(\mathcal{M})$ and $\mathcal{W}_{-}(\mathcal{M})$ for the example of section 7

$\mathcal{M}$, is located at $(p, q)=(0,0)$, and can be bounded by any $I_{1}$ and $I_{2}$ with $0<I_{1}<$ $1<I_{2}$. It is foliated by periodic orbits $p=q=0, I=$ constant, $\theta=(I-1) t+\theta_{0}$. The orbit at $I=1$ is a circle of equilibria, and the frequency $I-1$ passes through zero transversely there, so that the resonance Assumption 3 of section 2 is satisfied. The annulus $\mathcal{M}$ is connected to itself by a pair of three-dimensional homoclinic manifolds, $W_{+}(\mathcal{M})$ and $W_{-}(\mathcal{M})$, as is shown in Figure 18. The manifolds $W_{+}(\mathcal{M})$, and $W_{-}(\mathcal{M})$ are parametrized by $t, I$ and $\theta_{0}$ in the homoclinic solutions

$$
\begin{gathered}
p=p^{h}(t, I)=\mp \sqrt{2} \mu I \operatorname{sech}(\mu \sqrt{I} t) \tanh (\mu \sqrt{I} t), \\
q=q^{h}(t, I)= \pm \sqrt{2 I} \operatorname{sech}(\mu \sqrt{I} t), \\
I=I, \\
\theta=\theta^{h}(t, I)+\theta_{0}=(I-1) t-\mu \sqrt{I} \tanh (\mu \sqrt{I} t)+\theta_{0} .
\end{gathered}
$$

From (11.3d) we find the angle difference $\Delta \theta$ between the end points of any heteroclinic orbit connecting pairs of equilibria on $\mathcal{M}$ at $I=1$ to be $\Delta \theta=-2 \mu$.

Since the set $p=q=0$ is invariant even for nonzero $\varepsilon$, we can take the perturbed annulus $\mathcal{M}_{\varepsilon}$ to be the same as the annulus $\mathcal{M}$. At the resonance, $I=1$, the Melnikov function $M\left(I, \theta_{0}, \mu, \alpha, \beta, \gamma\right)$ on both homoclinic manifolds $W_{+}(\mathcal{M})$ and $W_{-}(\mathcal{M})$ is equal to

$$
M\left(1, \theta_{0}, \mu, \alpha, \beta, \gamma\right)=2 \sin \mu \sin \theta_{0}-\frac{4}{3} \alpha \mu+2 \beta \mu+\frac{8}{15} \gamma \mu^{3},
$$

see Kovačič [34, 35].

When $\mu$ is not a multiple of $\pi$, this Melnikov function has simple zeros in $\theta_{0}$ at some $\theta_{0}=\bar{\theta}_{0,1}$ and $\theta_{0}=\bar{\theta}_{0,2}=\pi-\bar{\theta}_{0,1}$, provided that

$$
\left|\frac{\mu}{\sin \mu}\left(\frac{2}{3} \alpha-\beta-\frac{4}{15} \gamma \mu^{2}\right)\right|<1 .
$$

Hence for all admissible $\alpha, \beta$, and $\gamma$, the stable and unstable manifolds $W^{s}\left(\mathcal{M}_{\varepsilon}\right)$ and $W^{u}\left(\mathcal{M}_{\varepsilon}\right)$ intersect transversely along two symmetric pairs of two-dimensional homoclinic surfaces, $\Sigma_{ \pm, \varepsilon}^{\alpha, \beta, \gamma}\left(\bar{\theta}_{0,1}\right)$ and $\Sigma_{ \pm, \varepsilon}^{\alpha, \beta, \gamma}\left(\bar{\theta}_{0,2}\right)$. In the Hamiltonian case, we find $\bar{\theta}_{0,1}=0$ and $\bar{\theta}_{0,2}=\pi$.

The restricted system (3.6) at the resonance $I=1$ for this example is

$$
h^{\prime}=-(1+\sqrt{\varepsilon} h) \sin \theta-\beta(1+\sqrt{\varepsilon}), \quad \theta^{\prime}=h-\sqrt{\varepsilon} \cos \theta,
$$




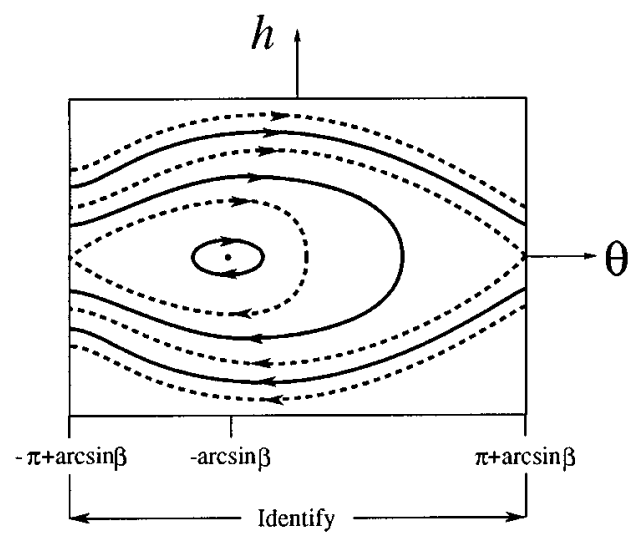

Figure 19. The phase portrait for the outer system of the example in section 7 in the dissipative case.

and the limiting outer system is

$$
h^{\prime}=-\sin \theta-\beta, \quad \theta^{\prime}=h .
$$

The rescaled Hamiltonian is

$$
\mathcal{H}(h, \theta)=\frac{1}{2} h^{2}-\cos \theta+\beta \theta,
$$

which is the Hamiltonian of the pendulum subject to a constant torque. In the nondissipative case, $\beta=0$, and the equations (11.7) describe the simple mathematical pendulum.

For the dissipative case, the phase portrait of the rescaled $h-\theta$ phase cylinder $\mathcal{M}_{0}$ of the equations (11.7) is shown in Figure 19. There are two equilibria on this phase cylinder, a center, $c_{0}$, at $(h, \theta)=(0,-\arcsin \beta)$, and a saddle, $s_{0}$, at $(h, \theta)=(0,-\pi+\arcsin \beta)$. The two branches of the stable and unstable manifolds, $\mathcal{W}^{s}\left(s_{0}\right)$ and $\mathcal{W}^{u}\left(s_{0}\right)$, to the right of the saddle $s_{0}$ coincide to form a separatrix that encloses a family of periodic orbits nested around the center. The two branches of the manifolds $\mathcal{W}^{s}\left(s_{0}\right)$ and $\mathcal{W}^{u}\left(s_{0}\right)$ to the left of the saddle $s_{0}$ wind around the cylinder $\mathcal{M}_{0}$ towards $h=+\infty$ and $h=-\infty$, respectively. For small positive $\sqrt{\varepsilon}$, the saddle $s_{0}$ persists as a saddle, $s_{\varepsilon}$, the center $c_{0}$ becomes a sink, $c_{\varepsilon}$, and the separatrix breaks. The top branch of the unstable manifold, $\mathcal{W}^{u}\left(s_{\varepsilon}\right)$, of the perturbed saddle $s_{\varepsilon}$ falls into the sink $c_{\varepsilon}$. No periodic orbits are left in this system, and all the points that lie in any compact domain that is all contained inside the unperturbed separatrix asymptote fall into the sink $c_{\varepsilon}$.

For the Hamiltonian case, there are again two equilibria on this phase cylinder, a center, $c_{0}$, at $(h, \theta)=(0,0)$ and a saddle, $s_{0}$, at $(h, \theta)=(0, \pi)$. The saddle $s_{0}$ is connected to itself by two (homoclinic) separatrices that enclose a family of periodic orbits, called libration orbits, nested around the center $c_{0}$. The two families of periodic orbits, called rotation orbits, above and below the libration region enclosed by the two separatrices wind once around the cylinder $\mathcal{M}_{0}$. All the features of the $h-\theta$ phase cylinder $\mathcal{M}_{0}$, including the two separatrices, are structurally stable and survive the addition of higher order terms in $\varepsilon$ to the constant $\mathcal{H}(h, \theta)$.

The inner system is

$$
\dot{p}=\mu^{2} q\left(1-q^{2}\right),
$$



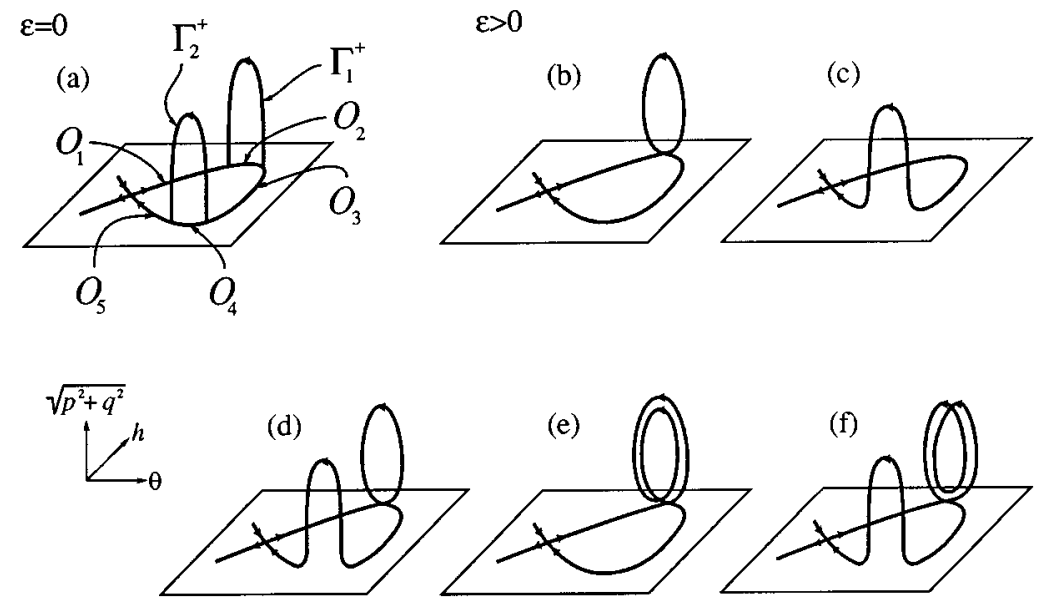

Figure 20. (a) The orbit segments comprising the $N$-bump singular transition orbits that connect the saddle $s_{0}$ to itself. (b) The one-bump orbit corresponding to the sequence $\mathrm{O}_{1} \mathrm{O}_{2} \mathrm{\Gamma}_{1}^{+} \mathrm{O}_{2} \mathrm{O}_{3} \mathrm{O}_{4} \mathrm{O}_{5}$. (c) The one-bump orbit corresponding to the sequence $\mathrm{O}_{1} \mathrm{O}_{2} \mathrm{O}_{3} \mathrm{\Gamma}_{2}^{+} \mathrm{O}_{5}$. (d) The two-bump orbit corresponding to the sequence $\mathrm{O}_{1} \mathrm{O}_{2} \mathrm{\Gamma}_{1}^{+} \mathrm{O}_{2} \mathrm{O}_{3} \mathrm{\Gamma}_{2}^{+} \mathrm{O}_{5}$. (e) The two-bump orbit corresponding to the sequence $\mathrm{O}_{1} \mathrm{O}_{2} \mathrm{\Gamma}_{1}^{+} \mathrm{O}_{2} \mathrm{\Gamma}_{1}^{+} \mathrm{O}_{2} \mathrm{O}_{3} \mathrm{O}_{4} \mathrm{O}_{5}$. (f) The threebump orbit corresponding to $\mathrm{O}_{1} \mathrm{O}_{2} \mathrm{\Gamma}_{1}^{+} \mathrm{O}_{2} \mathrm{\Gamma}_{1}^{+} \mathrm{O}_{2} \mathrm{O}_{3} \Gamma_{2}^{+} \mathrm{O}_{5}$.

$$
\begin{gathered}
\dot{q}=p, \\
\dot{h}=0, \\
\dot{\theta}=-\frac{1}{2} \mu^{2} q^{2} .
\end{gathered}
$$

In the phase space of this system, the two symmetric pairs of homoclinic intersection surfaces, $\Sigma_{ \pm, \varepsilon}^{\alpha, \beta, \gamma}\left(\bar{\theta}_{0,1}\right)$ and $\Sigma_{ \pm, \varepsilon}^{\alpha, \beta}\left(\bar{\theta}_{0,2}\right)$ (when the inequality (11.5) shows that they exist) collapse smoothly onto the pairs of surfaces, $\Sigma_{ \pm, 0}^{\alpha, \beta, \gamma}\left(\bar{\theta}_{0,1}\right)$ and $\sum_{ \pm, 0}^{\alpha, \beta, \gamma}\left(\bar{\theta}_{0,2}\right)$, parametrized by the expressions (11.3) with $I=1, \theta_{0}=\bar{\theta}_{0,1}$ or $\bar{\theta}_{0,2}$, and arbitrary $h$.

We now show how Theorem 1 applies to this example. In particular, we fix $0<\mu \ll \beta<1$ and $\tilde{\gamma}=\mu^{2} \gamma=\mathcal{O}(1)$. If $5 \alpha-2 \tilde{\gamma}=0$, then there exist two symmetric pairs of heteroclinic orbits on the singular intersection surfaces $\sum_{ \pm, 0}^{\alpha, \beta, \gamma}\left(\bar{\theta}_{0,1}\right)$, which connect two pairs of points on the separatrix on the $h-\theta$ cylinder $\mathcal{M}_{0}$, as shown in Figure 20. These two pairs of points cut the separatrix into five disjoint segments, denoted $O_{1}, \ldots, O_{5}$, where the numbers increase in the direction of increasing slow time $\tau$ along the separatrix. We denote the pair of heteroclinic orbits connecting the endpoints of the segment $O_{2}$ by $\Gamma_{1}^{ \pm}$, and the pair connecting the endpoints of the segment $O_{4}$ by $\Gamma_{2}^{ \pm}$.

We can now form a countable infinity of singular homoclinic orbits as follows. Consider any finite sequence $\sigma_{1}$ of pairs $\Gamma_{1}^{+} O_{2}$ and $\Gamma_{1}^{-} O_{2}$, including possibly the empty sequence. Moreover, let $\sigma_{2}$ be either $O_{4}, \Gamma_{2}^{+}$, or $\Gamma_{2}^{-}$. Define the sequence $S$ as 

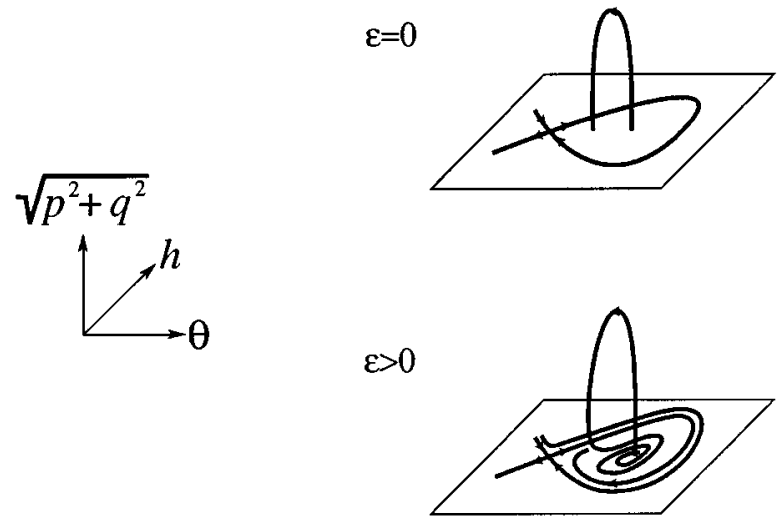

FIgURE 21. The one-bump Šilnikov orbit that connects the spiralsaddle $c_{\varepsilon}$ to itself.

$S=O_{1} O_{2} \sigma_{1} O_{3} \sigma_{2} O_{5}$, and exclude the sequence $O_{1} O_{2} O_{3} O_{4} O_{5}$ from this definition. If we concatenate the orbit segments $O_{1}, \ldots, O_{5}, \Gamma_{1}^{ \pm}$, and $\Gamma_{2}^{ \pm}$according to the sequence $S$, we obtain a singular orbit homoclinic to the point $s_{0}$, which we denote by $\Gamma_{0}^{S}$. If we denote by $-S$ the sequence obtained from $S$ by interchanging the + and - signs, then the corresponding singular homoclinic orbit $\Gamma_{0}^{-S}$ is the mirror image of the singular orbit $\Gamma_{0}^{S}$ under the transformation $(p, q) \mapsto(-p,-q)$, which preserves equations (11.1).

Theorem 1 now implies the following:

Proposition 11.1. Fix $0<\mu \ll \beta<1$ and $\tilde{\gamma}=\mu^{2} \gamma=\mathcal{O}(1)$. Let the sequence $S$ be as in the preceding paragraph, and $\Gamma_{0}^{S}$ its corresponding singular orbit homoclinic to the point $s_{0}$. Then, there exists a continuous function $\alpha_{S}(\varepsilon)$ with $5 \alpha_{S}(0)-2 \tilde{\gamma}=0$, such that for small positive $\varepsilon$ and $\alpha=\alpha_{S}(\varepsilon)$, there exists an orbit $\Gamma_{\varepsilon}^{S}$, homoclinic to the saddle $s_{\varepsilon}$. The orbit $\Gamma_{\varepsilon}^{S}$ is $\mathcal{O}(\delta(\varepsilon))$ close to the singular orbit $\Gamma_{0}^{S}$, where $\delta(\varepsilon)$ is a function with $\delta(\varepsilon) \rightarrow 0$ as $\varepsilon \rightarrow 0$. Moreover, $\alpha_{S}(\varepsilon)=\alpha_{-S}(\varepsilon)$, and the orbit $\Gamma_{\varepsilon}^{-S}$ is the mirror image of the orbit $\Gamma_{\varepsilon}^{S}$ under the transformation $(p, q) \mapsto(-p,-q)$.

By applying Theorem 2, we obtain the existence of multi-bump Šilnikov orbits. The demonstration of this fact proceeds as follows. As shown in Kovačič [35], when $0<\beta<1$ and the equation

$$
\alpha=\frac{3}{2}\left(1-\frac{\sin 2 \mu}{2 \mu}\right) \beta-\frac{3}{2} \frac{(1-\cos 2 \mu)}{2 \mu} \sqrt{1-\beta^{2}}+\frac{2}{5} \tilde{\gamma}
$$

is satisfied, the line $\theta=\bar{\theta}_{0,1}+\mu$ passes through the center $c_{0}$. The heteroclinic orbit emerging from $c_{0}$ lands inside the region enclosed by the separatrix homoclinic to the saddle $s_{0}$, for instance, when $0<\mu<\frac{\pi}{2}-\arcsin \beta$. When $\mu \ll 1$, equation (11.10) becomes $\alpha=\frac{2}{5} \tilde{\gamma}-\frac{3}{2} \mu \sqrt{1-\beta^{2}}+\mathcal{O}\left(\mu^{2}\right)$. For small nonzero $\sqrt{\varepsilon}$, this situation gives rise to a pair of single-bump Šilnikov orbits homoclinic to the spiral-saddle $c_{\varepsilon}$, see Figure 21.

Recall that each slow-time periodic orbit, which lies inside the region enclosed by the separatrix homoclinic to the saddle $s_{0}$ and intersects the line $\theta=\bar{\theta}_{0,1}+\mu$, in fact intersects this line in two points, one with positive and one with negative $h$. 
Therefore, there exist two pairs of heteroclinic orbits on the surfaces $\Sigma_{ \pm, \varepsilon}^{\alpha, \beta, \gamma}\left(\bar{\theta}_{0,1}\right)$ emanating from this periodic orbit, such that the $h$ coordinate along the two orbits of one of these pairs is positive, and the other negative. We denote each of these orbits by one of the four symbols $\Gamma_{ \pm}^{ \pm}$, where the sign in the subscript denotes the sign of the $h$ coordinate along this orbit, and the sign in the superscript denotes the one of the surfaces $\Sigma_{ \pm, \varepsilon}^{\alpha, \beta, \gamma}\left(\bar{\theta}_{0,1}\right)$ that this orbit lies on.

To obtain the existence of multi-bump Šilnikov orbits, we form the following type of a modified singular transition orbit. Its first segment is a heteroclinic orbit $\Gamma_{1}$ taking off at the center $c_{0} \equiv c_{1}$, and landing inside the region enclosed by the separatrix homoclinic to the saddle $s_{0}$ at the point $d_{2}$. The second segment is comprised of $\mathrm{N}_{2}$ revolutions around the slow-time periodic orbit $\mathrm{O}_{2}$ that passes through the point $d_{2}$. The last point of this segment is the point $c_{2}$ on the line $\theta=\bar{\theta}_{0,1}+\mu$, where the next segment, the heteroclinic orbit $\Gamma_{2}$, takes off. This orbit lands at the point $d_{3}$, which is again assumed to be inside the region enclosed by the separatrix homoclinic to the saddle $s_{0}$. This construction can continue for as long as the landing point $d_{i+1}$ of the $i$-th heteroclinic orbit $\Gamma_{i}$ is contained inside the region enclosed by the separatrix homoclinic to the saddle $s_{0}$. We denote the largest such $i$ by $K$. Modified singular transition orbits of the described type with $k$ bumps exist for all integers $k$ with $1 \leq k \leq K$. Each of the heteroclinic orbits $\Gamma_{i}$ can lie on either of the singular intersection surfaces $\Sigma_{ \pm, \varepsilon}^{\alpha, \beta, \gamma}\left(\bar{\theta}_{0,1}\right)$, and, if $i>1$, can have either positive or negative $h$ coordinate.

The maximal number of bumps, $K$, of the modified singular transition orbits described in the previous paragraph can be computed as a function of the parameters $\mu$ and $\beta$. In particular, we compute that the difference in the value of the expression $\frac{1}{2} h^{2}$ between any consecutive points $d_{i}$ and $c_{i}$ on this singular orbit must be equal to

$$
\frac{1}{2} h^{2}\left(c_{i}\right)-\frac{1}{2} h^{2}\left(d_{i}\right)=\sqrt{1-\beta^{2}}(1-\cos 2 \mu)+\beta(\sin 2 \mu-2 \mu) .
$$

On the other hand, the value of the expression $\frac{1}{2} h^{2}$ at the two points where the line $\theta=\bar{\theta}_{0,1}+\mu=-\arcsin \beta-2 \mu$ intersects the separatrix that encircles the center $c_{0}$ is

$$
\frac{1}{2} \bar{h}^{2}=\sqrt{1-\beta^{2}}(1+\cos 2 \mu)-\beta(\sin 2 \mu-2 \mu)+2 \beta\left(\arcsin \beta-\frac{\pi}{2}\right) .
$$

Therefore, $K$ must be the largest integer that satisfies the inequality

$$
\begin{aligned}
K & <\frac{\sqrt{1-\beta^{2}}(1+\cos 2 \mu)-\beta(\sin 2 \mu-2 \mu)+2 \beta\left(\arcsin \beta-\frac{\pi}{2}\right)}{\sqrt{1-\beta^{2}}(1-\cos 2 \mu)+\beta(\sin 2 \mu-2 \mu)}+1 \\
& =2 \frac{\sqrt{1-\beta^{2}}+\beta\left(\arcsin \beta-\frac{\pi}{2}\right)}{\sqrt{1-\beta^{2}}(1-\cos 2 \mu)+\beta(\sin 2 \mu-2 \mu)} .
\end{aligned}
$$

For $\mu \ll 1$, we have asymptotically

$$
K \approx \frac{1}{\mu^{2}}\left[1-\frac{\beta}{\sqrt{1-\beta^{2}}}\left(\frac{\pi}{2}-\arcsin \beta\right)\right] .
$$

(The expression in the square brackets is always positive because of the inequality $y / \tan y<1$, which is valid for all $0<y<\frac{\pi}{2}$.) 

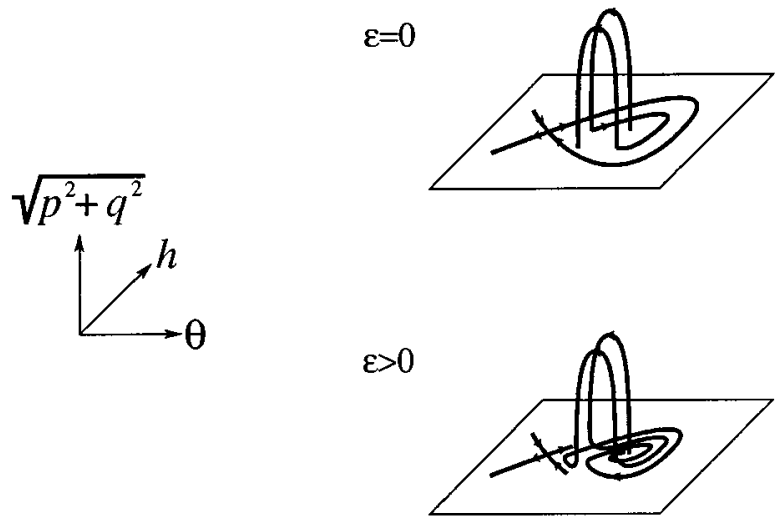

Figure 22. The two-bump Šilnikov orbit connecting the spiralsaddle $c_{\varepsilon}$ that corresponds to the sequence $\Gamma^{+} 0 \Gamma_{-}^{+}$.

Recall now the symbols $\Gamma_{ \pm}^{ \pm}$defined above. To each of the modified $k$-bump singular transition orbits discussed in the preceding two paragraphs, there corresponds a unique sequence $S$ composed of one of the symbols $\Gamma^{ \pm}$, followed by $(k-1)$ pairs $N_{i} \Gamma_{ \pm}^{ \pm}$with $i=2, \ldots, k$. Here $N_{i}$ are nonnegative integers. In turn, we denote by $\Gamma_{0}^{S}$ the singular transition orbit corresponding to the sequence $S$. We call $k$ the length of the sequence $S$. As in the case of singular orbits homoclinic to the point $s_{0}$, we denote by $-S$ the sequence obtained from $S$ by interchanging the + and - signs in the superscript. The corresponding singular homoclinic orbit $\Gamma_{0}^{-S}$ is then the mirror image of the singular orbit $\Gamma_{0}^{S}$ under the transformation $(p, q) \mapsto(-p,-q)$.

Theorem 2 now implies

Proposition 11.2. Fix $0<\beta<1,0<\mu<\frac{\pi}{2}-\arcsin \beta$, and $\tilde{\gamma}=\mu^{2} \gamma$. Let $K$ satisfy inequality (11.11), let the sequence $S$ of length $k$ with $1 \leq k \leq K$ be as in the preceding paragraph, and let $\Gamma_{0}^{S}$ be its corresponding modified singular transition orbit. Then, there exists a continuous function $\alpha_{S}(\varepsilon)$ with $\alpha_{S}(0)$ satisfying equation (11.10), such that, for small positive $\varepsilon$ and $\alpha=\alpha_{S}(\varepsilon)$, there exists an orbit $\Gamma_{\varepsilon}^{S}$, homoclinic to the spiral-saddle $c_{\varepsilon}$. Apart from the piece that spirals towards the point $c_{\varepsilon}$ near the $h-\theta$ annulus $\mathcal{M}_{\varepsilon}$, the orbit $\Gamma_{\varepsilon}^{S}$ is $\mathcal{O}(\delta(\varepsilon))$ close to the modified singular transition orbit $\Gamma_{0}^{S}$, where $\delta(\varepsilon)$ is a function with $\delta(\varepsilon) \rightarrow 0$ as $\varepsilon \rightarrow 0$. In particular, the orbit $\Gamma_{\varepsilon}^{S}$ makes $N_{i}$ full revolutions around the point $c_{\varepsilon}$ between its $(i-1)$-st and $i$-th bumps. Moreover, $\alpha_{S}(\varepsilon)=\alpha_{-S}(\varepsilon)$, and the orbit $\Gamma_{\varepsilon}^{-S}$ is the mirror image of the orbit $\Gamma_{\varepsilon}^{S}$ under the transformation $(p, q) \mapsto(-p,-q)$.

A two-bump orbit is shown in Figure 22. We remark that, if we substitute the right-hand side of equation (11.10) for $\alpha$ in the inequality (11.5), this inequality is always satisfied.

Two general remarks seem appropriate at this point. The first remark concerns the asymptotic nature of Propositions 11.1 and 11.2. In particular, even though Proposition 11.1 implies the existence of a countable family of multi-bump orbits homoclinic to the saddle $s_{\varepsilon}$, it makes no claim about the existence of all the members of this family for a given fixed value of $\varepsilon>0$. In fact, only the existence of a finite number of these orbits can be guaranteed in this case, and this number may decrease 
with increasing $\varepsilon$. A similar statement is true for the family of Šilnikov orbits homoclinic to the spiral saddle $c_{\varepsilon}$ discussed in Proposition 11.2. As $\varepsilon \rightarrow 0$, orbits in that family can have at most a finite number, $K$, of bumps, but the numbers of revolutions, $N_{i}$, between the consecutive bumps of the members of this family can be as large as we please. Again, for a given fixed value of $\varepsilon>0$, only the existence of a finite number of these multi-bump Šilnikov orbits can be guaranteed, so that the numbers of revolutions, $N_{i}$, may have a uniform upper bound that depends on the value of $\varepsilon$. Notice, however, that Šilnikov orbits with any number of bumps between one and $K$ must exist for any small enough but still fixed $\varepsilon>0$.

The second remark concerns the relation of the multi-bump orbits described in Propositions 11.1 and 11.2 to another frequently discussed type of homoclinic orbits with multiple excursions, the so-called multi-loop orbits. The existence of these latter orbits follows from return-map techniques, such as the ones yielding the multiloop orbits described in Glendinning and Sparrow [15]. The difference between these multi-loop orbits and the multi-bump orbits given by our Propositions 11.1 and 11.2 is in that each multi-loop orbit must necessarily return to a small neighborhood of the equilibrium point to which it is homoclinic, while this is not true for any of the multi-bump orbits. In fact, between two bumps, each such multi-bump orbit must stay a finite distance away from the equilibrium point to which it is homoclinic. Because of this fact, the existence of the multi-bump homoclinic orbits described in Propositions 11.1 and 11.2 does not seem to follow in any obvious way from a returnmap approach. However, the return-map technique of Glendinning and Sparrow [15] may be used to show the existence of an infinite sequence of multi-loop subsidiary orbits corresponding to each of the multi-bump Šilnikov orbits homoclinic to the spiral-saddle $c_{\varepsilon}$ described in Proposition 11.2.

A proposition somewhat similar to Proposition 11.2 establishing the existence of multi-bump heteroclinic connections between the saddle $s_{\varepsilon}$ and the spiral-saddle $c_{\varepsilon}$ can be deduced from Theorem 3. Note that in this case the connections exist in an open set of parameter values.

Finally, we take a look at the Hamiltonian case. For simplicity, we take $0<\mu<$ $\pi$. The structure of the limiting inner and outer systems is discussed in Kovačič $[33,36]$. In particular, the two limiting intersection surfaces $\Sigma_{ \pm, 0}^{0,0,0}(0)$ intersect the $h-\theta$ cylinder $\mathcal{M}_{0}$ along the lines $\theta=-\mu$ and $\theta=\mu$, and the two limiting intersection surfaces $\Sigma_{ \pm, 0}^{0,0,0}(\pi)$ intersect the $h-\theta$ cylinder $\mathcal{M}_{0}$ along the lines $\theta=$ $\pi-\mu$ and $\theta=\pi+\mu \equiv-\pi+\mu$. All the libration orbits of the equations (11.7) that extend beyond $\theta=-\mu$ and $\theta=\mu$ intersect these two lines transversely in $\mathcal{M}_{0}$. Thus, each of these libration orbits is connected to itself by two pairs of heteroclinic orbits on $\Sigma_{ \pm, 0}^{0,0,0}(0)$ : one at $h$ and another at $-h$. Each of the libration orbits that extend beyond $\theta=-\pi+\mu$ and $\theta=\pi-\mu$ is connected to itself by two pairs of heteroclinic orbits on $\Sigma_{ \pm, 0}^{0,0,0}(\pi)$. In particular, if $\kappa=\max \{\mu, \pi-\mu\}$, each libration orbit that extends beyond $\theta=-\kappa$ and $\theta=\kappa$ is connected to itself by four pairs of homoclinic orbits, two pairs at $h$ and two pairs at $-h$. Each of the rotation orbits is connected to itself by one pair of heteroclinic orbits lying on the surfaces $\Sigma_{ \pm, 0}^{0,0,0}(0)$, and by one pair of heteroclinic orbits lying on the surfaces $\Sigma_{ \pm, 0}^{0,0,0}(\pi)$.

We now restrict the values of $\mu$ further to $0<\mu<\pi / 2$, and consider those libration orbits of the outer system (11.7), which are connected to themselves by four pairs of heteroclinic orbits. These orbits form a family $O_{0}(\mathcal{H})$, with $\cos \mu<$ $\mathcal{H}<1$, where $\mathcal{H}$ denotes the value of the rescaled Hamiltonian (11.8) with $\beta=0$ 


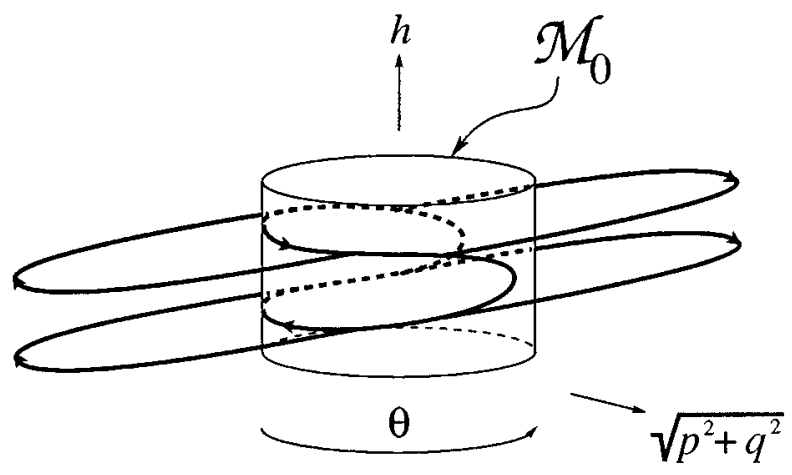

Figure 23. The end points of its corresponding four heteroclinic orbits cut the libration orbit $O_{0}(\mathcal{H})$ into eight segments. One can form singular transition orbits out of these segments and the four heteroclinic orbits by following the prescription described prior to Proposition 11.3.

along this orbit. The end points of its corresponding four heteroclinic orbits cut the libration orbit $O_{0}(\mathcal{H})$ into eight segments $O_{1}(\mathcal{H}), \ldots, O_{8}(\mathcal{H})$, see Figure 23. We let $O_{1}(\mathcal{H})$ denote the segment of the orbit $O_{0}(\mathcal{H})$ with $-\mu<\theta<\mu$ and $h>0$, and string the other segments along in the direction of increasing slow time, $\tau$. We denote the heteroclinic orbits corresponding to the slow-time orbit $O_{0}(\mathcal{H})$ by $\Gamma_{1}^{ \pm}(\mathcal{H}), \Gamma_{2}^{ \pm}(\mathcal{H}), \Gamma_{3}^{ \pm}(\mathcal{H})$, and $\Gamma_{4}^{ \pm}(\mathcal{H})$, where the numbering proceeds in the direction of decreasing $h$, and the + or - denotes the sign of the $q$ coordinate along that orbit. Note that the orbits $\Gamma_{1}^{ \pm}(\mathcal{H})$ and $\Gamma_{4}^{ \pm}(\mathcal{H})$ lie on the singular one-bump surface $\Sigma_{ \pm, 0}^{0,0,0}(0)$, and $\Gamma_{2}^{ \pm}(\mathcal{H})$ and $\Gamma_{3}^{ \pm}(\mathcal{H})$ lie on $\Sigma_{ \pm, 0}^{0,0,0}(\pi)$.

We define $S$ to be an admissible sequence of symbols $O_{1}, \ldots, O_{8}, \Gamma_{1}^{ \pm}, \ldots, \Gamma_{4}^{ \pm}$ if it is finite, begins with some $\Gamma_{i}^{ \pm}$and ends with some (possibly different) $\Gamma_{j}^{ \pm}$, and any successive pair of symbols in the sequence can only be chosen from among $O_{i} O_{i+1}, O_{8} O_{1}, O_{4} \Gamma_{4}^{ \pm}, O_{6} \Gamma_{3}^{ \pm}, O_{7} \Gamma_{2}^{ \pm}, O_{1} \Gamma_{1}^{ \pm}, \Gamma_{1}^{ \pm} O_{1}, \Gamma_{2}^{ \pm} O_{3}, \Gamma_{3}^{ \pm} O_{4}$, and $\Gamma_{4}^{ \pm} O_{6}$. Any admissible sequence $S$ corresponds to a singular orbit, $\Gamma_{0}^{S}(\mathcal{H})$, homoclinic to the slow-time periodic orbit $O_{0}(\mathcal{H})$. As before, if we denote by $-S$ the sequence obtained from $S$ by interchanging the + and - signs, then the corresponding singular homoclinic orbit $\Gamma_{0}^{-S}(\mathcal{H})$ is the mirror image of the singular orbit $\Gamma_{0}^{S}(\mathcal{H})$ under the transformation $(p, q) \mapsto(-p,-q)$.

Theorem 4, and the extension theorems of Kaplun (Lagerstrom [41]) and Eckhaus [10] now imply

Proposition 11.3. For all small enough $\varepsilon$, some function $\eta(\varepsilon)>0$ with $\eta(\varepsilon) \rightarrow$ 0 as $\varepsilon \rightarrow 0$, every unperturbed orbit $O_{0}(\mathcal{H})$ with $\cos \mu+\eta(\varepsilon)<\mathcal{H}<1-\eta(\varepsilon)$, and every admissible sequence $S$, there exists a perturbed periodic orbit $O_{\varepsilon}(\mathcal{H})$ a distance $\mathcal{O}(\sqrt{\varepsilon})$ away from $O_{0}(\mathcal{H})$ and an orbit $\Gamma_{\varepsilon}^{S}(\mathcal{H})$ which is homoclinic to the orbit $O_{\varepsilon}(\mathcal{H})$. Here the value of the perturbed constant $\mathcal{H}_{\varepsilon}$ along the orbit $O_{\varepsilon}(\mathcal{H})$ is the same as the value of $\mathcal{H}$ along $O_{0}(\mathcal{H})$. Moreover, apart from the pieces that asymptote onto the orbit $O_{\varepsilon}(\mathcal{H})$ in forward and backward time, the orbit $\Gamma_{\varepsilon}^{S}(\mathcal{H})$ is $\mathcal{O}(\delta(\varepsilon))$ close to the singular orbit $\Gamma_{0}^{S}(\mathcal{H})$, where $\delta(\varepsilon)$ is a function with $\delta(\varepsilon) \rightarrow 0$ 
as $\varepsilon \rightarrow 0$. Finally, the orbit $\Gamma_{\varepsilon}^{-S}(\mathcal{H})$ is the mirror image of the orbit $\Gamma_{\varepsilon}^{S}(\mathcal{H})$ under the transformation $(p, q) \mapsto(-p,-q)$.

The existence of the homoclinic orbits described in this proposition also follows from the standard arguments that use the lambda lemma (Palis [50]). These same arguments also show that the multi-bump orbits exist for all $\varepsilon$ for which the onebump orbits exist.

Results similar to those described in Proposition 11.3 are valid for the libration orbits that are connected to themselves by two pairs of heteroclinic orbits, and also the rotation orbits.

\section{ACKNOWLEDGMENT}

We thank R. Camassa, C. K. R. T. Jones, N. Kopell, M. Levi, C. Soto-Treviño and S. K. Tin for helpful discussions. We thank the Theoretical and Computing Divisions and the Center for Nonlinear Science at the Los Alamos National Laboratory for their hospitality and support during the summers of 1992 and 1993. T. K. gratefully acknowledges support from the National Science Foundation through Grant No. DMS-9307074 and from the Alfred Sloan Foundation through a Sloan Research Fellowship. G. K. gratefully acknowledges support from the U.S. D.O.E. through Grant No. DE-FG02-93ER25154, the National Science Foundation through Grant Nos. DMS-9403750 and DMS-9502142 (CAREER grant), and the Alfred Sloan Foundation through a Sloan Research Fellowship.

\section{REFERENCES}

1. D. V. Anosov [1967]. Geodesic flows on closed Riemannian manifolds of negative curvature, Proc. Steklov Inst. Math. 90. MR 39:3527 (and 36:7157)

2. V. I. Arnold [1964]. Instability of dynamical systems with many degrees of freedom, Sov. Math. Dokl. 5, 581-585. MR 29:329

3. V. I. Arnold [1965]. Proof of a theorem of A. N. Kolmogorov on the invariance of quasiperiodic motions under small perturbations of the Hamiltonian, Uspekhi Mat. Nauk 18, no. 5, 13-41; English transl., Russian Math. Surveys 18, no. 5, 9-36. MR 29:328

4. V. I. Arnold, V.V. Kozlov, and A. I. Neihstadt (Eds.) [1988], Dynamical Systems III, in Encyclopedia of Mathematical Sciences, Vol. 3, Springer-Verlag: Berlin, Heidelberg, New York, London, Paris, Tokyo, 1993. MR 95d:58043b

5. S. Aubry and P. Y. LeDaeron [1983]. The discrete Frenkel-Kontorova model and its extensions, I. Exact results for ground states, Physica D 8, 381-422. MR 85f:58032

6. S. N. Chow, J. Hale, and J. Mallet-Paret [1980]. An example of bifurcation to homoclinic orbits, J. Diff. Eqns. 37, 351-373. MR 81 m:58056

7. W. A. Coppel [1978]. Dichotomies in stability theory, Lecture Notes in Mathematics, vol. 629, Springer-Verlag, New York. MR 58:1332

8. S. P. Diliberto [1960]. Perturbation theorems for periodic surfaces I, Rend. Cir. Mat. Palermo, Ser. 2 9, 265-299. MR 26:420a

9. S. P. Diliberto [1961]. Perturbation theorems for periodic surfaces II, Rend. Cir. Mat. Palermo, Ser. 2 10, 111-161. MR 26:420b

10. W. Eckhaus [1973]. Matched Asymptotic Expansions and Singular Perturbations, NorthHolland, Amsterdam. MR 58:32369

11. N. Fenichel [1971]. Persistence and smoothness of invariant manifolds for flows, Ind. Univ. Math. J. 21, 193-225. MR 44:4313

12. N. Fenichel [1974]. Asymptotic stability with rate conditions, Ind. Univ. Math. J. 23, 11091137. MR 49:4036

13. N. Fenichel [1977]. Asymptotic stability with rate conditions, II, Ind. Univ. Math. J. 26, 81-93. MR 54:14002

14. N. Fenichel [1979]. Geometric singular perturbation theory for ordinary differential equations, J. Diff. Eqns. 31, 53-98. MR 80m:58032 
15. P. Glendinning and C. Sparrow [1984]. Local and global behavior near homoclinic orbits, $J$. Stat. Phys. 35, 645-696. MR 86h:58109

16. J. Gruendler [1985]. The existence of homoclinic orbits and the method of Melnikov for systems in $\mathbb{R}^{n}$, SIAM J. Math. Anal. 16, 907-931. MR 87e:58069

17. J. Guckenheimer and P. J. Holmes [1983]. Nonlinear Oscillations, Dynamical Systems, and Bifurcations of Vector Fields, Springer-Verlag: New York, Heidelberg, Berlin. MR 85f:58002

18. J. Hadamard [1901]. Sur l'itération et les solutions asymptotiques des équationes différentielles, Bull. Soc. Math. France, 28, 224-228.

19. J. K. Hale [1961]. Integral manifolds of perturbed differential systems, Ann. Math. 73, 496531. MR 23:A1108

20. G. Haller and S. Wiggins [1993]. Orbits homoclinic to resonances: the Hamiltonian case, Physica D 66, 298-346. MR 94i:58149

21. G. Haller and S. Wiggins [1995]. $N$-pulse homoclinic orbits in perturbations of hyperbolic manifolds of Hamiltonian equilibria, Arch. Rat. Mech. Anal. 130, 25-101.

22. M. W. Hirsch, C. C. Pugh, and M. Shub [1970]. Invariant manifolds, Bull. Amer. Math. Soc. 76, 1015-1019. MR 45:1188

23. M. W. Hirsch, C. C. Pugh, and M. Shub [1977]. Invariant Manifolds, Springer Lecture Notes in Mathematics, vol. 583. Springer Verlag: New York, Heidelberg, Berlin. MR 58:18595

24. P. J. Holmes [1979]. A nonlinear oscillator with a strange attractor, Phil. Trans. Roy. Soc. London Ser. A 292, 419-448. MR 81e:58036

25. P. J. Holmes and J. E. Marsden [1982a]. Horseshoes in perturbation of Hamiltonian systems with two degrees of freedom, Comm. Math. Phys. 82, 523-544. MR 83a:58070

26. P. J. Holmes and J. E. Marsden [1982b]. Melnikov's method and Arnold diffusion for perturbations of integrable Hamiltonian systems, J. Math. Phys. 23, 669-675. MR 84h:58055

27. J. Jarnik and J. Kurzweil [1969]. On invariant sets and invariant manifolds of differential systems, J. Diff. Eqns. 6, 247-263. MR 40:2970

28. C. K. R. T. Jones and N. Kopell [1994]. Tracking invariant manifolds with differential forms in singularly perturbed systems, J. Diff. Eqns. 108, 64-88. MR 95c:34085

29. C. K. R. T. Jones, T. J. Kaper, and N. Kopell [1996]. Tracking invariant manifolds up to exponentially small errors, SIAM J. Math. Anal. 27, 558-577. CMP 1966:9

30. A. Katok [1982]. Some remarks on Birkhoff and Mather twist map theorems, Erg. Th. Dyn. Sys. 2, 185-194. MR 84m:58041

31. A. Katok and B. Hasselblatt [1995]. Introduction to the modern theory of smooth dynamical systems, Cambridge University Press. CMP 1995:11

32. A. N. Kolmogorov [1954]. On conservation of conditionally periodic motion for a small change in Hamilton's function, Dokl. Akad. Nauk. SSSR 98:4, 525-530; English transl. in his Selected Works, Vol. I: Mathematics and Mechanics, Kluwer, Dordrecht, 1991, pp. 349-354. MR 16:924c, 93d:01096

33. G. Kovačič [1992]. Hamiltonian dynamics of orbits homoclinic to a resonance band, Phys. Lett. A 167, 137-142. MR 93k:34100

34. G. Kovačič [1992]. Dissipative dynamics of orbits homoclinic to a resonance band, Phys. Lett. A 167, 143-150. MR 93k:34101

35. G. Kovačič [1995]. Singular perturbation theory for homoclinic orbits in a class of nearintegrable dissipative systems, SIAM J. Math. Anal. 26, 1611-1643. CMP 1996:3

36. G. Kovačič [1993]. Singular perturbation theory for homoclinic orbits in a class of nearintegrable Hamiltonian systems, J. Dyn. Diff. Eqns. 5, 559-597. MR 95c:58153

37. G. Kovačič and S. Wiggins [1992]. Orbits homoclinic to resonances with an application to chaos in a model of the forced and damped Sine-Gordon equation, Physica D 57, 185-225. MR 93f: 58153

38. I. Kupka [1964]. Stabilité des variétés invariantes d'un champ de vecteurs pour les petites perturbations, Comp. Rend. Acad. Sci. Paris 258, 4197-4200. MR 28:5238

39. J. Kurzweil [1968]. Invariant sets of differential systems, Differentsial'nye Uravneniya 4, no. 5, 785-797. (Russian) MR 38:370

40. W. T. Kyner [1961]. Invariant manifolds, Rend. Cir. Mat. Palermo, Ser. 2 10, 98-110. MR 26:6534

41. P. A. Lagerstrom [1988]. Matched Asymptotic Expansions: Ideas and Techniques, SpringerVerlag: New York, Heidelberg, Berlin. MR 89g:34081 
42. L. M. Lerman and Ia. L. Umanski [1984]. On the existence of separatrix loops in fourdimensional systems similar to integrable Hamiltonian systems, Prikl. Mat. Mekh. 47, 335340; English transl., J. Appl. Math. Mech. 47, 335-340. MR 86b:58043

43. X.-B. Lin [1989]. Shadowing lemma and singularly-perturbed boundary-value problems, SIAM J. Appl. Math. 49(1), 26-54. MR 90a:34126

44. J. N. Mather [1982]. Existence of quasi-periodic orbits for twist homeomorphisms of the annulus, Topology 21, 457-467. MR 84g:58084

45. J. N. Mather [1985]. More Denjoy minimal sets for area-preserving diffeomorphisms, Comment Math. Helv. 60, 508-557. MR 87f:58086

46. V. K. Melnikov [1963]. On the stability of the center for time periodic perturbations, Trans. Moscow Math. Soc. 12, 1-57. MR 27:5981

47. J. K. Moser [1962]. On invariant curves of area preserving mappings of an annulus, Nachr. Akad. Wiss. Göttingen Math. Phys. K1. II, 1-20. MR 26:5255

48. J. K. Moser [1966]. A rapidly converging iteration method and nonlinear partial differential equations, I, Ann. Scuola Norm. Sup. Pisa, Ser. III 20, 265-315. MR 33:7667

49. J. K. Moser [1966]. A rapidly converging iteration method and nonlinear partial differential equations, II, Ann. Scuola Norm. Sup. Pisa, Ser. III 20, 499-535. MR 34:6280

50. J. Murdock [1988]. Qualitative theory of nonlinear resonance by averaging and dynamical systems methods, Dynamics Reported 1, 91-172. MR 89h:58166

51. J. Palis [1969]. On Morse-Smale dynamical systems, Topology 8, 385-405. MR 39:7620

52. K. J. Palmer [1984]. Exponential dichotomies and transversal homoclinic points, J. Diff. Eqns. 55, 225-256. MR 86d:58088

53. O. Perron [1922]. Über die Gestalt der Integralkurven einer Differentialgleichung erster Ordnung in der Umgebung eines singulären Punktes, Math. Zeit. 15, 121-146.

54. H. Poincaré [1886] Mémoire sur les courbes définies par une équation differentielle IV, J. Math. Pures Appl. 4, no. 2, 151-217

55. H. Poincaré [1899]. Les Méthodes Nouvelles de la Mécanique Celeste, 3 vols., Gauthier-Villars: Paris.

56. C. Robinson [1983]. Sustained resonance for a nonlinear system with slowly varying coefficients, SIAM J. Math. Anal. 14, 847-860. MR 85e:70027

57. C. Robinson [1988]. Horseshoes for autonomous Hamiltonian systems using the Melnikov integral, Ergod. Th. Dynam. Sys. 8*, 395-409. MR 89m:58100

58. R. J. Sacker [1965]. A new approach to the perturbation theory of invariant surfaces, Comm. Pure Appl. Math. 18, 717-732. MR 32:6003

59. R. J. Sacker [1969]. A perturbation theorem for invariant manifolds and Hölder continuity, $J$. Math. Mech. 18, 705-762. MR 39:578

60. R. J. Sacker and G. R. Sell [1978]. A spectral theory for linear differential systems, J. Dyn. Diff. Eqns. 27, 320-358. MR 58:18604

61. K. Sakamoto [1990]. Invariant Manifolds In Singular Perturbation Problems For Ordinary Differential Equations, Proc. Royal Soc. Edinburgh, A-Math. 116, 45-78. MR 91j:34088

62. G. Sotomayor [1966]. Structural stability of first order and Banach varieties, Univ. Nac. Ingen. Inst. Mat. Puras Apl. Notas Mat., 4, 11-52 (Portuguese). MR 40:6594

63. S. K. Tin [1994]. Ph.D. thesis, Brown University.

64. S. K. Tin [1994]. On the dynamics of tangent spaces near a normally hyperbolic invariant manifold, preprint.

65. S. K. Tin, N. Kopell, and C. K. R. T. Jones [1994]. Invariant manifolds and singularly perturbed boundary value problems, SIAM J. Numer. Anal. 31, 1558-1576. CMP 1995:4

66. S. Wiggins [1988]. Global Bifurcations and Chaos: Analytical Methods, Springer-Verlag: New York. MR 89m:58057

Department of Mathematics, Boston University, Boston, Massachusetts 02215

E-mail address: tasso@math.bu.edu

Mathematical Sciences Department, Rensselaer Polytechnic Institute, Troy, New YORK 12180

E-mail address: kovacg@rpi.edu 